Geometry \& Topology

Volume 8 (2004) 335-412

Published: 14 February 2004

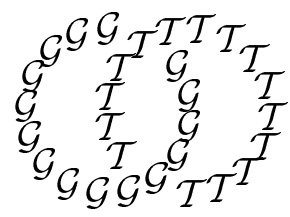

\title{
Formal groups and stable homotopy of commutative rings
}

\author{
Stefan SchWEde \\ Mathematisches Institut, Universität Bonn \\ 53115 Bonn, Germany \\ Email: schwede@math.uni-bonn.de
}

\begin{abstract}
We explain a new relationship between formal group laws and ring spectra in stable homotopy theory. We study a ring spectrum denoted $D B$ which depends on a commutative ring $B$ and is closely related to the topological André-Quillen homology of $B$. We present an explicit construction which to every 1-dimensional and commutative formal group law $F$ over $B$ associates a morphism of ring spectra $F_{*}: H \mathbb{Z} \longrightarrow D B$ from the Eilenberg-MacLane ring spectrum of the integers. We show that formal group laws account for all such ring spectrum maps, and we identify the space of ring spectrum maps between $H \mathbb{Z}$ and $D B$. That description involves formal group law data and the homotopy units of the ring spectrum $D B$.
\end{abstract}

\section{AMS Classification numbers Primary: 55U35}

Secondary: 14L05

Keywords: Ring spectrum, formal group law, André-Quillen homology

Proposed: Bill Dwyer

Seconded: Thomas Goodwillie, Haynes Miller
Received: 12 July 2003

Revised: 12 February 2004 


\section{Introduction}

In this paper we explain a new relationship between formal group laws and ring spectra in stable homotopy theory. We use formal group laws to construct maps of ring spectra and describe spaces of ring spectrum maps in terms of formal group data.

Our main object of study is a ring spectrum denoted $D B$ which functorially depends on a commutative ring $B$. The ring spectrum $D B$ controls the stable homotopy theory of augmented commutative $B$-algebras. Its homotopy groups $\pi_{*} D B$ are the Cartan-Bousfield-Dwyer algebra of stable homotopy operations of commutative simplicial $B$-algebras [10, 7, 12. The homotopy groups $\pi_{*} D B$ are also isomorphic to the $\Gamma-$ homology [42], relative to $B$, of the polynomial algebra $B[x]$ on one generator, and to the topological André-Quillen homology [1] of the associated Eilenberg-MacLane spectra. Both $\Gamma$-homology and topological André-Quillen homology arise in obstruction theory for $E_{\infty}$ ring spectrum structures [41. We elaborate more on the precise relationship in Remark 3.3 below, and we also refer to the survey paper [3] by Basterra and Richter.

We present an explicit construction which to every 1-dimensional and commutative formal group law $F$ over $B$ associates a homomorphism of ring spectra

$$
F_{*}: H \mathbb{Z} \longrightarrow D B
$$

from the Eilenberg-MacLane ring spectrum of the integers to $D B$. We prove that in this way formal group laws account for all ring spectrum maps, ie, we show:

Theorem The construction which sends a 1-dimensional, commutative formal group law $F$ to the ring spectrum map $F_{*}$ induces a natural bijection between the set of strict isomorphism classes of formal group laws over $B$ and the set of homotopy classes of ring spectrum maps from $H \mathbb{Z}$ to $D B$.

This theorem is a corollary of the identification of the space of all ring spectrum maps between the Eilenberg-MacLane ring spectrum $H \mathbb{Z}$ and $D B$. That description involves the homotopy units $(D B)^{\times}$of the ring spectrum $D B$. The group-like simplicial monoid $(D B)^{\times}$of homotopy units acts by conjugation on the space $\operatorname{Ring}(H \mathbb{Z}, D B)$ of ring spectrum maps. The construction $F_{*}$ of the first theorem extends to a natural weak map from the classifying space of the groupoid of formal group laws and strict isomorphisms $\mathcal{F} \mathcal{G} \mathcal{L}^{\operatorname{str}}(B)$ to the homotopy orbit space $\operatorname{Ring}(H \mathbb{Z}, D B) /$ conj. of the conjugation action of the 
identity component $(D B)_{1}^{\times}$of the units on the space of ring spectrum maps. In Theorem 5.2 we show

Theorem The weak map

$$
\mathcal{F} \mathcal{G L}^{\operatorname{str}}(B) \longrightarrow \operatorname{Ring}(H \mathbb{Z}, D B) / \text { conj. }
$$

from the classifying space of the groupoid of formal group laws and strict isomorphisms to the homotopy orbit space of the conjugation action is a weak homotopy equivalence.

Another corollary of this main theorem is that the ring spectrum $D B$ is not equivalent to the Eilenberg-MacLane ring spectrum of any differential graded algebra (or, equivalently, of any simplicial ring); see Corollary 13.2 for the precise statement. This should be compared to the fact that the spectrum underlying $D B$ (ie, ignoring the multiplication) is stably equivalent to the smash product $H \mathbb{Z} \wedge H B$ (see Theorem 3.2 (b)), in particular it is equivalent to a product of Eilenberg-MacLane spectra. Corollary 13.2 says that the multiplicative structure of $D B$ is considerably more complicated.

Prerequisites We freely use the language and standard results from the theory of model categories; the original source for this material is [39], a more modern introduction can be found in [13, and the ultimate reference is currently [23]. Our notion of ring spectrum is that of a Gamma-ring (see [29, 2.13] or [44, Def. 2.1]). Gamma-rings are based on a symmetric monoidal smash product for $\Gamma$-spaces with good homotopical properties [48, 8, 29]. The foundational material about the homotopy theory of Gamma-rings and their modules can be found in [44]; a summary is also given in Section 2]. The results of this paper can be translated into other frameworks for ring spectra by the general comparison procedures described in 32,45$]$. We also need a few basic facts from the theory of formal group laws, which in this paper (with the exception of Section 13) are always 1-dimensional and commutative; all we need is contained in 28] or in Chapter III, $\S 1$ of [19].

\section{Outline of the paper}

Section 2 We review some general facts about $\Gamma$-spaces and Gamma-rings. We recall the assembly map (2.5) from the smash product to the composition product of $\Gamma$-spaces which is used several times in this paper.

Section 3 We review the Gamma-ring $D B$ and summarize some of its properties in Theorem [3.2. Construction 3.5 associates a homomorphism of ring 
spectra $F_{*}: H \mathbb{Z} \longrightarrow D B$ to every formal group law $F$. We state in Theorem 3.8 how this accounts for all homotopy classes of Gamma-ring maps.

Section 4 For every Gamma-ring $R$ we construct a natural conjugation action of the simplicial monoid of homotopy units $R^{\times}$(3.9) on $R$ through Gamma-ring homomorphisms.

Section [5] We construct a weak map

$$
\mathcal{F} \mathcal{G} \mathcal{L}^{\operatorname{str}}(B) \stackrel{\sim}{\longleftarrow} \widetilde{\mathcal{F G}}^{\operatorname{str}}(B) \stackrel{\kappa}{\longrightarrow} \operatorname{Ring}(H \mathbb{Z}, D B) / \text { conj. }
$$

from the classifying space of the groupoid of formal group laws and strict isomorphisms to the homotopy orbit space of the conjugation action of Section 4. The left map is a weak equivalence by construction. Theorem 5.2 which is the main theorem of this paper, says that the right map $\kappa$ is a weak equivalence.

Section 6 We use a filtration of the Gamma-ring $D B$, coming from powers of the augmentation ideal, to reduce the proof of Theorem 5.2 to showing that a truncated version

$$
\kappa_{k}: \widetilde{\mathcal{B u d}}^{k}(B) \longrightarrow \operatorname{Ring}\left(H \mathbb{Z}, D_{k} B\right) / \text { conj. }
$$

of the map $\kappa$ of Section [5 is a weak equivalence for all $k \geq 1$ (see Theorem 6.4). Here $\widetilde{\mathcal{B u d}}^{k}(B)$ is (weakly equivalent to) the classifying space of the groupoid of $k$-buds (or $k$-jets) of formal group laws and $D_{k} B$ is the "quotient" Gammaring of $D B$ by the "ideal" coming from $(k+1)$ st powers of the augmentation ideal.

Section 17 We exploit that two successive stages in the filtration of $D B$ form a "singular extension" of ring spectra

$$
\left(B \otimes \mathcal{S}^{k}\right)^{!} \longrightarrow D_{k} B \longrightarrow D_{k-1} B
$$

where $\left(B \otimes \mathcal{S}^{k}\right)$ ! is a "square zero ideal" coming from the $k$-th symmetric power functor. This allows us to reduce the problem to showing that a certain map

$$
\kappa_{B \otimes \mathcal{S}^{k}}: \tilde{\mathcal{Z}}\left(B \otimes \mathcal{S}^{k}\right) \longrightarrow \operatorname{der}\left(H \mathbb{Z}, B \otimes \mathcal{S}^{k}\right) / \text { conj. }
$$

to the homotopy orbit space of the derivations of $H \mathbb{Z}$ with coefficients in the symmetric power functor is a weak equivalence. Here $\widetilde{\mathcal{Z}}\left(B \otimes \mathcal{S}^{k}\right)$ is (weakly equivalent to) the classifying space of the groupoid of symmetric 2-cocycles of degree $k$ over $B$. 
Section 8 For later use we define a map

$$
\lambda_{G}: \mathcal{Z}(G) \longrightarrow \operatorname{map}_{\mathcal{G R}}\left(H \mathbb{Z}^{\mathrm{c}}, H \mathbb{Z} \times G_{s t}^{!}\right)_{h G_{s t}(\mathbb{Z})}
$$

for every functor $G$ from the category of finitely generated free abelian groups to the category of all abelian groups; the important case is when $G$ is a symmetric power functor $B \otimes \mathcal{S}^{k}$. Here $\mathcal{Z}(G)$ is the classifying space of the groupoid of symmetric 2-cocycles of the functor $G$ (8.1), $\operatorname{map}_{\mathcal{G R}}$ denotes the simplicial mapping space of Gamma-rings, $G_{s t}$ is the Dold-Puppe stabilization of $G$ (8.9) and the Gamma-ring $H \mathbb{Z} \times G_{s t}^{!}$is the split singular extension of $H \mathbb{Z}$ by the bimodule $G_{s t}^{!}$(7.4).

Section 9 We compare the map $\kappa_{B \otimes \mathcal{S}^{k}}$ of Section 7 with the map $\lambda_{B \otimes \mathcal{S}^{k}}$ of Section 8 by means of a commutative square

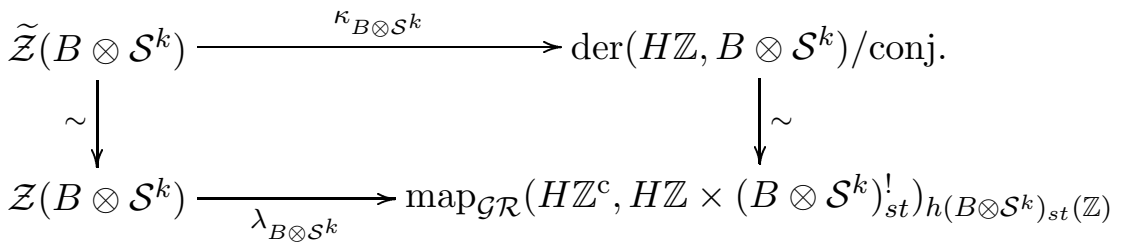

in which the vertical maps are weak equivalences. Hence instead of showing that $\kappa_{B \otimes \mathcal{S}^{k}}$ is a weak equivalence we may show that $\lambda_{B \otimes \mathcal{S}^{k}}$ is.

Section 10 By the results of the previous sections, the proof of the main theorem is reduced to an identification of the space of Gamma-ring maps

$$
\operatorname{map}_{\mathcal{G R}}\left(H \mathbb{Z}^{\mathrm{c}}, H \mathbb{Z} \times\left(B \otimes \mathcal{S}^{k}\right)_{s t}^{!}\right)
$$

(or more precisely a certain homotopy orbit space thereof) with the classifying space of symmetric 2-cocycles. In this section we reinterpret the above mapping space in terms of the category $s \mathcal{F}$ of simplicial functors from the category of finitely generated free abelian groups to the category of abelian groups. We note that the construction which sends $G \in s \mathcal{F}$ to the split extension $H \mathbb{Z} \times G^{!}$ (17.4) has a left adjoint

$$
\mathcal{L}: \mathcal{G} R / H \mathbb{Z} \longrightarrow s \mathcal{F}
$$

from the category of Gamma-rings over $H \mathbb{Z}$ to the category of simplicial functors. Moreover, the two functors form a Quillen adjoint pair between model categories. In order to identify the above mapping space, we evaluate the left adjoint $\mathcal{L}$ on the Gamma-ring $H \mathbb{Z}^{\mathrm{c}}$, the cofibrant replacement of $H \mathbb{Z}$. We denote by $J$ the functor which supports the universal symmetric 2-cocycle (8.3). The main result of this section, Theorem 10.2 states that the map

$$
\mathcal{L}\left(H \mathbb{Z}^{\mathrm{c}}\right) \longrightarrow J
$$


which is adjoint to the "universal derivation" (8.6)

$$
\left(1, d_{u}\right): H \mathbb{Z}^{\mathrm{c}} \longrightarrow H \mathbb{Z} \times J^{!}
$$

is a stable equivalence of simplicial functors. This implies that for any reduced functor $G$ the homotopy groups of the space

$$
\operatorname{map}_{\mathcal{G R}}\left(H \mathbb{Z}^{\mathrm{c}}, H \mathbb{Z} \times G_{s t}^{!}\right) \cong \operatorname{map}_{s \mathcal{F}}\left(\mathcal{L}\left(H \mathbb{Z}^{\mathrm{c}}\right), G_{s t}\right)
$$

are isomorphic to the hyper-cohomology groups $\operatorname{Ext}_{\mathcal{F}}^{*}(J, Q G)$, for $* \leq 0$, in the abelian category $\mathcal{F}$ of reduced functors from the category of finitely generated abelian groups to the category of abelian groups. The chain complex $Q G$ is MacLane's cubical construction for the functor $G$ (8.9).

Section 11 We prove a homological criterion, Theorem 11.1, in terms of the functor $G$ for when the map $\lambda_{G}$ defined in Section 8 is a weak equivalence. Loosely speaking, the criterion requires that "MacLane cohomology equals topological Hochschild cohomology" for the functor $G$, compare Remark 11.2 The precise meaning of this is that for all integers $m \leq 2$ the map

$$
\operatorname{Ext}_{\mathcal{F}}^{m}(I, G) \longrightarrow \mathbb{E x t}_{\mathcal{F}}^{m}(I, Q G)
$$

be an isomorphism. The map $G \longrightarrow Q G$ is a model for Dold-Puppe stabilization and it is initial, in the derived category of $\mathcal{F}$, among maps from $G$ to complexes whose homology functors are additive. $\operatorname{Ext}_{\mathcal{F}}^{m}(I,-)$ denotes hyperExt groups of the functor $I$ with coefficients in a chain complex of functors. In Example 11.3 we also give a functor for which the criterion fails.

Section 12 In Theorem 12.1 we verify the homological criterion of Theorem 11.1 for the symmetric power functors $G=B \otimes \mathcal{S}^{k}$. This finishes the proof of our main theorem: Theorem 11.1 shows that the map $\lambda_{B \otimes \mathcal{S}^{k}}$ is a weak equivalence, hence by the commutative square (9.1) the map $\kappa_{B \otimes \mathcal{S}^{k}}$ is a weak equivalence. The commutative diagram (7.7) of fibre sequences shows inductively that the maps $\kappa_{k}: \widetilde{\mathcal{B u d}}^{k}(B) \longrightarrow \operatorname{Ring}\left(H \mathbb{Z}, N_{k}\right) /$ conj. of Theorem 6.4 are weak equivalences. Hence the (weak) map $\kappa: \mathcal{F G} \mathcal{L}^{\operatorname{str}}(B) \longrightarrow \operatorname{Ring}(H \mathbb{Z}, D B) /$ conj. of Theorem 5.2 is a weak equivalence.

Section 13 In the last section we give an application of the main theorem as well as an outlook towards possible generalizations and future directions. Variations of the main construction are possible, and some of them are described in this final section. For example, formal groups can be replaced by formal $A-$ modules where $A$ is any ring (not necessarily commutative). Such formal $A$ modules give rise to Gamma-ring maps from the Eilenberg-MacLane Gammaring of $A$ into $D B$. Furthermore when considering higher dimensional formal 
group laws, the natural target of the construction is a matrix Gamma-ring over $D B$ of the corresponding dimension.

Acknowledgments The author would like to thank Jeff Smith for suggesting the method used in Section 4 to obtain the conjugation action; moreover, many ideas that appear in Section 10 arose in a joint project with Smith on derivations of ring spectra.

\section{Review of $\Gamma$-spaces and Gamma-rings}

In this section we review some general facts about $\Gamma$-spaces and Gamma-rings, and we fix notation and terminology. None of this material is new, but we present it in a form which is convenient for this paper. We also prove certain properties of the assembly map (2.5) from the smash product to the composition product of $\Gamma$-spaces which are used several times in this paper.

$2.1 \Gamma$-spaces The category of $\Gamma$-spaces was introduced by Segal 48, who showed that it has a homotopy category equivalent to the stable homotopy category of connective spectra. Bousfield and Friedlander [8] considered a bigger category of $\Gamma$-spaces in which the ones introduced by Segal appeared as the special $\Gamma$-spaces (2.3). Their category admits a closed simplicial model category structure with a notion of stable weak equivalences giving rise again to the homotopy category of connective spectra. Then Lydakis [29] introduced internal function objects and a symmetric monoidal smash product with good homotopical properties.

The category $\Gamma^{\mathrm{op}}$ has one object $n^{+}=\{0,1, \ldots, n\}$ for every non-negative integer $n$, and morphisms are the maps of sets which send 0 to $0 . \Gamma^{\mathrm{op}}$ is equivalent to the opposite of Segal's category $\Gamma$ [48. A $\Gamma$-space is a covariant functor from $\Gamma^{\mathrm{op}}$ to the category of simplicial sets taking $0^{+}$to a one point simplicial set. A morphism of $\Gamma$-spaces is a natural transformation of functors. We denote by $\mathbb{S}$ the $\Gamma$-space which takes $n^{+}$to $n^{+}$, considered as a constant simplicial set. If $X$ is a $\Gamma$-space and $K$ a pointed simplicial set, a new $\Gamma$-space $X \wedge K$ is defined by setting $(X \wedge K)\left(n^{+}\right)=X\left(n^{+}\right) \wedge K$.

A $\Gamma$-space $X$ can be prolonged, by direct limit, to a functor from the category of finite pointed sets to the category of (not necessarily finite) pointed sets. By degreewise evaluation and formation of the diagonal of the resulting bisimplicial sets, it can furthermore be promoted to a functor from the category of pointed simplicial sets to itself $[8, \S 4]$. The extended functor preserves weak equivalences 
of simplicial sets [8, Prop. 4.9] and is automatically simplicial, ie, it comes with coherent natural maps $K \wedge X(L) \longrightarrow X(K \wedge L)$. We will not distinguish notationally between the prolonged functor and the original $\Gamma$-space.

The homotopy groups of a $\Gamma$-space $X$ are defined as

$$
\pi_{n} X=\operatorname{colim}_{i} \pi_{n+i}\left|X\left(S^{i}\right)\right|,
$$

where the colimit is formed using the maps

$$
S^{1} \wedge X\left(S^{n}\right) \longrightarrow X\left(S^{1} \wedge S^{n}\right) \text {. }
$$

A map of $\Gamma$-spaces is a stable equivalence if it induces isomorphisms on homotopy groups. Since the functor given by a prolonged $\Gamma$-space preserves connectivity [8, 4.10], the homotopy groups of a $\Gamma$-space are always trivial in negative dimensions.

2.2 Smash products In [29 Thm. 2.2], Lydakis defines a smash product for $\Gamma$-spaces which is characterized by the universal property that $\Gamma$-space maps $X \wedge Y \longrightarrow Z$ are in bijective correspondence with maps

$$
X\left(k^{+}\right) \wedge Y\left(l^{+}\right) \quad \longrightarrow \quad Z\left(k^{+} \wedge l^{+}\right)
$$

which are natural in both variables. By [29, Thm. 2.18], the smash product of $\Gamma$-spaces is associative and commutative with unit $\mathbb{S}$, up to coherent natural isomorphism. There are also internal homomorphism $\Gamma$-spaces [29, 2.1], adjoint to the smash-product, so that the category of $\Gamma$-spaces forms a symmetric monoidal closed category.

2.3 Special $\Gamma$-spaces A $\Gamma$-space $X$ is called special if the map $X\left(k^{+} \vee l^{+}\right)$ $\longrightarrow X\left(k^{+}\right) \times X\left(l^{+}\right)$induced by the projections from $k^{+} \vee l^{+}$to $k^{+}$and $l^{+}$is a weak equivalence for all $k$ and $l$. In this case, the weak map

$$
X\left(1^{+}\right) \times X\left(1^{+}\right) \stackrel{\sim}{\longleftarrow} X\left(2^{+}\right) \stackrel{X(\nabla)}{\longrightarrow} X\left(1^{+}\right)
$$

induces an abelian monoid structure on $\pi_{0}\left(X\left(1^{+}\right)\right)$. Here $\nabla: 2^{+} \longrightarrow 1^{+}$is the fold map defined by $\nabla(1)=1=\nabla(2)$. $X$ is called very special if it is special and the monoid $\pi_{0}\left(X\left(1^{+}\right)\right)$is a group. By Segal's theorem (48, Prop. 1.4], see also [8, Thm. 4.2]), the spectrum associated to a very special $\Gamma$-space $X$ is an $\Omega$-spectrum in the sense that the maps $\left|X\left(S^{n}\right)\right| \longrightarrow \Omega\left|X\left(S^{n+1}\right)\right|$ adjoint to the spectrum structure maps are homotopy equivalences. In particular, the homotopy groups of a very special $\Gamma$-space $X$ are naturally isomorphic to the homotopy groups of the simplicial set $X\left(1^{+}\right)$. 
2.4 Model structures Bousfield and Friedlander introduce two model category structures for $\Gamma$-spaces called the strict and the stable model categories [8, 3.5, 5.2]. It will be more convenient for our purposes to work with slightly different model category structures, which we called the Quillen- or Q-model category structures in [4] and [46]. The strict and stable Q-structures have the same weak equivalences, hence the same homotopy categories, as the corresponding Bousfield-Friedlander model category structures. In this paper we never consider the Bousfield-Friedlander model structures, so we drop the decoration ' $Q$ ' for the other model structure.

We call a map of $\Gamma$-spaces a strict fibration (resp. a strict equivalence) if it is a Kan fibration (resp. weak equivalence) of simplicial sets when evaluated at every $n^{+} \in \Gamma^{\mathrm{op}}$. Cofibrations are defined as the maps having the left lifting property with respect to all strict acyclic fibrations. The cofibrations can be characterized as the injective maps with projective cokernel, see 44, Lemma A3 (b)] for the precise statement. By [39, II.4 Thm. 4], the strict equivalences, strict fibrations and cofibrations make the category of $\Gamma$-spaces into a closed simplicial model category.

More important is the stable model category structure. This one is obtained by localizing the strict model structure with respect to the stable equivalences. We call a map of $\Gamma$-spaces a stable fibration if it has the right lifting property with respect to the cofibrations which are also stable equivalences. By 44, Thm. 1.5], the stable equivalences, stable fibrations and cofibrations make the category of $\Gamma$-spaces into a closed simplicial model category. A $\Gamma$-space $X$ is stably fibrant if and only if it is very special and $X\left(n^{+}\right)$is fibrant as a simplicial set for all $n^{+} \in \Gamma^{\mathrm{op}}$.

A $\Gamma$-space $X$ defines a spectrum $X(S)$ (in the sense of [8, Def. 2.1]) whose $n$-th term is the value of the prolonged $\Gamma$-space at $S^{n}$. For example, the $\Gamma$-space $\mathbb{S}$ becomes isomorphic to the identity functor of the category of pointed simplicial sets after prolongation. So the associated spectrum is the sphere spectrum. The functor that sends a $\Gamma$-space $X$ to the spectrum $X(S)$ has a right adjoint [8. Lemma 4.6], and these two functors form a Quillen pair. One of the main theorems of [8] says that this Quillen pair induces an equivalence between the homotopy category of $\Gamma$-spaces, taken with respect to the stable equivalences, and the stable homotopy category of connective spectra (see [8, Thm. 5.8]). We do not use this result here, but it is the main motivation for the study of $\Gamma$-spaces.

2.5 The assembly map Given two $\Gamma$-spaces $X$ and $Y$, there is a natural map $X \wedge Y \longrightarrow X \circ Y$ from the smash product to the composition product 29, 
2.12], 46, 1.8]. The formal and homotopical properties of this assembly map are of importance to this paper. Since $\Gamma$-spaces prolong to functors defined on the category of pointed simplicial sets, they can be composed. Explicitly, for $\Gamma$-spaces $X$ and $Y$, we set $(X \circ Y)\left(n^{+}\right)=X\left(Y\left(n^{+}\right)\right)$. This composition $\circ$ is a monoidal (though not symmetric monoidal) product on the category of $\Gamma$-spaces. The unit is the same as for the smash product, it is the $\Gamma$-space $\mathbb{S}$ which as a functor is the inclusion of $\Gamma^{\mathrm{op}}$ into all pointed simplicial sets.

The assembly map is obtained as follows. Prolonged $\Gamma$-spaces are naturally simplicial functors [8, $\S 3]$, which means that there are natural coherent maps $X(K) \wedge L \longrightarrow X(K \wedge L)$. This simplicial structure gives maps

$$
X\left(n^{+}\right) \wedge Y\left(m^{+}\right) \longrightarrow X\left(n^{+} \wedge Y\left(m^{+}\right)\right) \longrightarrow X\left(Y\left(n^{+} \wedge m^{+}\right)\right)
$$

natural in both variables. From this the assembly map $X \wedge Y \longrightarrow X \circ Y$ is obtained by the universal property of the smash product of $\Gamma$-spaces. The assembly map is associative and unital, $\mathbb{S}$ being the unit for both $\wedge$ and $\circ$. In technical terms: the identity functor on the category of $\Gamma$-spaces becomes a lax monoidal functor from $(\mathcal{G} S, \wedge)$ to $(\mathcal{G} S, \circ)$. The homotopical properties of smash and composition product and of the assembly map are summarized in the following theorem, which is due to Lydakis [29].

Theorem 2.6 (a) The composition product of $\Gamma$-spaces preserves stable equivalences in each of its variables.

(b) The smash product with a cofibrant $\Gamma$-space preserves stable equivalences.

(c) Let $X$ and $Y$ be $\Gamma$-spaces, one of which is cofibrant. Then the assembly map

$$
X \wedge Y \longrightarrow X \circ Y
$$

is a stable equivalence.

Proof Parts (b) and (c) are [29, Prop. 5.12] and 29, Prop. 5.23] respectively.

For any $\Gamma$-space $F$ the structure map $S^{1} \wedge F\left(S^{n}\right) \longrightarrow F\left(S^{1} \wedge S^{n}\right)$ is $(2 n+1)$ connected [29, Prop. 5.21]. So the map $\pi_{*} \Omega^{n}\left|F\left(S^{n}\right)\right| \longrightarrow \pi_{*} F$ is an isomorphism for $*<n$. Hence if $F \longrightarrow F^{\prime}$ is a stable equivalence of $\Gamma$-spaces, then the map $F\left(S^{n}\right) \longrightarrow F^{\prime}\left(S^{n}\right)$ is $2 n$-connected. If $X$ is any $\Gamma$-space, then the prolonged functor preserves connectivity [29, Prop. 5.20], so the map $X\left(F\left(S^{n}\right)\right) \longrightarrow X\left(F^{\prime}\left(S^{n}\right)\right)$ is also $2 n$-connected. Thus the map $X \circ F \longrightarrow X \circ F^{\prime}$ is a stable equivalence. 
It remains to show that the map $F \circ X \longrightarrow F^{\prime} \circ X$ is also a stable equivalence. By the previous paragraph we may assume that $X$ is cofibrant, and then the claim follows from parts (b) and (c).

2.7 Gamma-rings and their modules Our notion of ring spectrum is that of a Gamma-ring. Gamma-rings are the monoids in the symmetric monoidal category of $\Gamma$-spaces with respect to the smash product and they are special cases of 'Functors with Smash Product' (FSPs, cf. [5, 1.1], 37, 2.2]). One can describe Gamma-rings as 'FSPs defined on finite sets'. From a Gamma-ring one obtains an FSP or symmetric ring spectrum (24, [4, 2.1.11]) by prolongation and, in the second case, evaluation on spheres. A more detailed discussion of the homotopy theory of Gamma-rings can be found in 44. Homotopy theoretic results about the Gamma-rings can be translated into other frameworks for ring spectra by the general comparison procedures of 32 .

Explicitly, a Gamma-ring is a $\Gamma$-space $R$ equipped with maps

$$
\mathbb{S} \longrightarrow R \text { and } R \wedge R \longrightarrow R,
$$

called the unit and multiplication map, which satisfy certain associativity and unit conditions (see [31, VII.3]). A morphism of Gamma-rings is a map of $\Gamma$-spaces commuting with the multiplication and unit maps. If $R$ is a Gammaring, a left $R$-module is a $\Gamma$-space $N$ together with an action map $R \wedge N \longrightarrow N$ satisfying associativity and unit conditions (see again [31, VII.4]). A morphism of left $R$-modules is a map of $\Gamma$-spaces commuting with the action of $R$. We denote the category of left $R$-modules by $R$-mod.

One similarly defines right modules and bimodules. Because of the universal property of the smash product of $\Gamma$-spaces (2.2), Gamma-rings are in bijective correspondence with lax monoidal functors from the category $\Gamma^{\mathrm{op}}$ to the category of pointed simplicial sets (both under smash product).

2.8 Examples The unit $\mathbb{S}$ of the smash product is a Gamma-ring in a unique way. The category of $\mathbb{S}$-modules is isomorphic to the category of $\Gamma$-spaces. Other standard examples of Gamma-rings are monoid rings over the sphere Gamma-ring $\mathbb{S}$ and Eilenberg-MacLane models of classical rings. If $M$ is a simplicial monoid, we define a $\Gamma$-space $\mathbb{S}[M]$ by

$$
\mathbb{S}[M]\left(n^{+}\right)=M^{+} \wedge n^{+}
$$

(so $\mathbb{S}[M]$ is isomorphic, as a $\Gamma$-space, to $\mathbb{S} \wedge M^{+}$, see (2.1)). There is a unit map $\mathbb{S} \longrightarrow \mathbb{S}[M]$ induced by the unit of $M$ and a multiplication map $\mathbb{S}[M] \wedge \mathbb{S}[M] \longrightarrow \mathbb{S}[M]$ induced by the multiplication of $M$ which turn $\mathbb{S}[M]$ 
into a Gamma-ring. This construction of the monoid ring over $\mathbb{S}$ is left adjoint to the functor which takes a Gamma-ring $R$ to the simplicial monoid $R\left(1^{+}\right)$.

If $A$ is an ordinary ring, then the Eilenberg-MacLane $\Gamma$-space $H A$ is given by the functor which takes a pointed set $K$ to the reduced free $A$-module $\widetilde{A}[K]$ generated by $K$. The unit map

$$
\eta: K \longrightarrow \widetilde{A}[K]
$$

is the inclusion of generators. The multiplication map

$$
\mu: \widetilde{A}[K] \wedge \widetilde{A}[L] \longrightarrow \widetilde{A}[K \wedge L]
$$

takes a smash product $\left(\sum_{k \in K} a_{k} \cdot k\right) \wedge\left(\sum_{l \in L} b_{l} \cdot l\right)$ to the element $\sum a_{k} b_{l} \cdot(k \wedge l)$. For later reference we note that the multiplication $\mu: H A \wedge H A \longrightarrow H A$ factors as the composition

$$
H A \wedge H A \stackrel{\text { assembly }}{\longrightarrow} H A \circ H A \stackrel{\text { eval. }}{\longrightarrow} H A
$$

of the assembly map (2.5) and the evaluation map.

More examples of Gamma-rings arise from algebraic theories and as endomorphism Gamma-rings, see [46, 4.5, 4.6]. The Gamma-ring $D B$ (3.1) is such an example.

The modules over a fixed Gamma-ring and the category of all Gamma-rings form simplicial model categories, created by the forgetful functor to $\Gamma$-spaces [44. Thm. 2.2 and Thm. 2.5]. In these model structures a map is a weak equivalence (resp. fibration) if it is a stable equivalence (resp. stable fibration) as a map of $\Gamma$-spaces. For a ring $A$, the Eilenberg-MacLane functor $H$ is the right adjoint of a Quillen equivalence between the model category of $H A$ modules and the model category of simplicial $A$-modules [44, Thm. 4.4].

\section{The Gamma-ring $D B$ and formal group laws}

In this section we recall the definition and some basic properties of our main object of study, the Gamma-ring $D B$, for $B$ a fixed commutative ring. This Gamma-ring parameterizes the stable homotopy theory of augmented commutative simplicial $B$-algebras. In Construction 3.5 we explain how a formal group law $F$ over $B$ gives rise to a homomorphism of ring spectra $F_{*}: H \mathbb{Z} \longrightarrow D B$. The rest of this paper is then devoted to studying the homotopical significance of that construction. 
By "parameterizing the stable homotopy theory" we mean that there is a Quillen-equivalence between the model category of $D B$-modules and the model category of spectra of simplicial commutative $B$-algebras, see Theorem $3.2(\mathrm{~d})$. Commutative simplicial algebras have been the object of much study [38, 12, 20, 21, 43, 51]. The homology theory arising as the derived functor of abelianization in this category is known as André-Quillen homology for commutative rings. The stable homotopy category of simplicial commutative $B$-algebras is equivalent to the homotopy category of $D B$-modules. The homotopy groups of $D B$ are the coefficients of the universal homology theory for commutative algebras.

3.1 The Gamma-ring $D B$ The $\Gamma$-space underlying the Gamma-ring $D B$ takes a pointed set $K$ to the augmentation ideal of the power series ring generated by $K$, considered as a constant simplicial set:

$$
D B(K)=\operatorname{kernel}(\widetilde{B} \llbracket K \rrbracket \longrightarrow \widetilde{B} \llbracket * \rrbracket=B) .
$$

The tilde over $\widetilde{B} \llbracket K \rrbracket$ indicates that the power series generator corresponding to the basepoint of $K$ has been set equal to 0 ; thus $\widetilde{B} \llbracket * \rrbracket$ reduces to the coefficient ring.

The product which makes $D B$ into a Gamma-ring comes from substitution of power series. To define the multiplication map $\mu: D B \wedge D B \longrightarrow D B$ we need to describe a natural associative map

$$
\mu: D B(K) \wedge D B(L) \longrightarrow D B(K \wedge L)
$$

for pointed sets $K$ and $L$. An element of $D B(K)$ is represented by a power series $f$ in variables $K$ without constant term. Similarly, an element of $D B(L)$ is represented by a power series $g$ in variables $L$. The multiplication map $\mu$ takes the smash product

$$
f \wedge g \in D B(K) \wedge D B(L)
$$

to the power series $\mu(f \wedge g)$ in the variables $K \wedge L$ defined by

$$
\begin{aligned}
\mu\left(f\left(k_{1}, \ldots, k_{m}\right)\right. & \left.\wedge g\left(l_{1}, \ldots, l_{n}\right)\right)= \\
& f\left(g\left(k_{1} \wedge l_{1}, \ldots, k_{1} \wedge l_{n}\right), \ldots, g\left(k_{m} \wedge l_{1}, \ldots, k_{m} \wedge l_{n}\right)\right) .
\end{aligned}
$$

The unit map $\eta: \mathbb{S} \longrightarrow D B$, ie, natural transformation

$$
\eta: K \longrightarrow D B(K)
$$

sends an element of $K$ to the generator it represents. The multiplication and unit transformations $\mu$ and $\eta$ are associative and unital because substitution of power series is, so $D B$ is in fact a Gamma-ring. 
Some important properties of $D B$ are summarized in the following theorem. Most of these results are compiled from [46]. In [46, 7.9], we use the notation $D B$ for a slightly different Gamma-ring, namely the Gamma-ring associated to the algebraic theory of augmented commutative $B$-algebras. Let $D B^{\text {pol }}$ denote the sub- $\Gamma$-space of $D B$ whose value at a pointed set $K$ consists of all polynomials in $K$, ie, the power series in $D B(K)$ with only finitely many non-zero coefficients. The sub- $\Gamma$-space $D B^{\text {pol }}$ is closed under the multiplication of $D B$, and the unit map $\eta: \mathbb{S} \longrightarrow D B$ factors through $D B^{\text {pol }}$. Hence $D B^{\text {pol }}$ is a Gamma-ring and the inclusion $D B^{\text {pol }} \longrightarrow D B$ is a homomorphism. $D B^{\text {pol }}$ is exactly the Gamma-ring which is denoted $D B$ in [46, 7.9]; there is no homotopical difference between the two Gamma-rings since the inclusion $D B^{\text {pol }} \longrightarrow D B$ is a stable equivalence, see Theorem 3.2 (a) below. However, in this paper it is more convenient to work with the power series model, so we give it the simpler name.

Theorem 3.2 (a) The inclusion $D B^{\text {pol }} \longrightarrow D B$ is a stable equivalence of Gamma-rings.

(b) As a $\Gamma$-space, $D B$ is stably equivalent to the derived smash product of the Eilenberg-MacLane $\Gamma$-spaces $H \mathbb{Z}$ and $H B$; in particular, the stable homotopy groups of $D B$ are additively isomorphic to the spectrum homology of the Eilenberg-MacLane spectrum of $B$.

(c) The graded ring of homotopy groups of $D B$ is isomorphic to the ring of stable homotopy operations of commutative augmented simplicial $B-$ algebras.

(d) There is a Quillen adjoint functor pair between the model category of $D B$-modules and the model category $\mathcal{S} p(B$-alg $)$ of spectra of commutative augmented simplicial $B$-algebras. The adjoint pair passes to an equivalence between the homotopy category of $D B$-modules and the homotopy category of connective spectra of commutative augmented simplicial $B$-algebras.

Proof (a) Let $\left(B \otimes \mathcal{S}^{k}\right)$ ! denote the $\Gamma$-space defined by

$$
\left(B \otimes \mathcal{S}^{k}\right)^{!}(K)=B \otimes\left(\widetilde{\mathbb{Z}}[K]^{\otimes k} / \Sigma_{k}\right),
$$

the tensor product of $B$ with the $k$-th symmetric power of the reduced free abelian group on $K$. An isomorphic description of $\left(B \otimes \mathcal{S}^{k}\right)^{!}(K)$ is as the free reduced $B$-module on the $k$-th symmetric power of $K$,

$$
\left(B \otimes \mathcal{S}^{k}\right)^{!}(K) \cong \widetilde{B}\left[K^{\wedge k} / \Sigma_{k}\right]
$$


The underlying $\Gamma$-space of $D B$ is the infinite product of the symmetric power functors $\left(B \otimes \mathcal{S}^{k}\right)^{!}$for all $k \geq 1$. The polynomial model $D B^{\text {pol }}$ is the weak product of these symmetric power functors. Since the stable homotopy groups of the $\Gamma$-space $\left(B \otimes \mathcal{S}^{k}\right)$ ! are trivial up to dimension $2 k-3$ [11, 12.3], the weak product and the product are stably equivalent.

(b) We let $S P$ denote the $\Gamma$-space which takes a pointed set $K$ to its infinite symmetric product, ie, the free abelian monoid generated by $K$ with basepoint as identity element. By the Dold-Thom theorem, the group completion map $S P \longrightarrow H \mathbb{Z}$ is a stable equivalence of $\Gamma$-spaces. We choose a cofibrant replacement $H B^{\mathrm{c}}$ of $H B$ as a $\Gamma$-space and obtain a chain of homomorphisms of $\Gamma$-spaces

$$
\begin{aligned}
& H B^{\mathrm{c}} \wedge H \mathbb{Z} \stackrel{\sim}{\longleftarrow} H B^{\mathrm{c}} \wedge S P \stackrel{\sim}{\longrightarrow} H B^{\mathrm{c}} \circ S P \\
& \stackrel{\sim}{\longrightarrow} H B \circ S P \cong D B^{\mathrm{pol}} \stackrel{\sim}{\longrightarrow} D B .
\end{aligned}
$$

The first map is a stable equivalence since smashing with a cofibrant $\Gamma$-space preserves stable equivalences [29, 5.12]. The second map is the assembly map (2.5), which is a stable equivalence by [29, 5.23]. The third map is a stable equivalence since the composition product of $\Gamma$-spaces preserves stable equivalences in both variables (Theorem 2.6 (a)). The reduced free $B$-module generated by $S P(K)$ is isomorphic to the polynomials without constant term generated by $K$, subject to the basepoint relation. This gives as isomorphism of $\Gamma$-spaces between $H B \circ S P$ and $D B^{\text {pol }}$. The last map is a stable equivalence by part (a).

Part (c) and (d) are special cases of [46, 4.11]. and [46, 4.4] respectively; see also [46, 7.9].

The ring of stable homotopy operations of commutative augmented simplicial $B$-algebras - ie, the graded ring of homotopy groups of $D B$ - is sometimes called the stable Cartan-Bousfield-Dwyer-algebra since these authors calculated the unstable operations for $B=\mathbb{F}_{p}$, see [10, 7, 12]. An explicit calculation of $\pi_{*} D \mathbb{F}_{p}$ can be found as Theorems 12.3 (for $p=2$ ) and 12.6 (for $p$ odd) in Bousfield's unpublished paper [7]. The fact that the ring of stable homotopy operations is generally not commutative shows that $D B$ is not stably equivalent to the derived smash product $H B \wedge^{L} H \mathbb{Z}$ as a Gamma-ring (unless $B$ is a $\mathbb{Q}$-algebra).

\subsection{Relation to topological André-Quillen homology and $\Gamma$-homology}

There are isomorphisms of graded abelian groups

$$
\pi_{*} D B \cong H \Gamma_{*}(B[x] \mid B ; B) \cong T A Q_{*}(H B[x] \mid H B ; H B) .
$$


Here $H \Gamma_{*}(B[x] \mid B ; B)$ is the $\Gamma-$ homology in the sense of Robinson and Whitehouse [42, of the polynomial algebra $B[x]$, considered as an augmented $B$ algebra; moreover, $T A Q_{*}(H B[x] \mid H B ; H B)$ denotes the topological AndréQuillen homology [1] of the Eilenberg-MacLane ring spectrum $H B[x]$ relative to $H B$. Both $\Gamma$-homology and topological André-Quillen homology groups are studied because they carry obstructions to the existence of $E_{\infty}$ ring spectrum structures 41] (it is a coincidence that the symbol $\Gamma$ occurs both in $\Gamma$-homology and as the category $\left.\Gamma^{\mathrm{op}}\right)$.

The first isomorphism in (3.4) comes about as follows. By 3.2 (a) above, the Gamma-ring $D B$ has a stably equivalent polynomial model $D B^{\text {pol }}$; as a $\Gamma-$ space, $D B^{\text {pol }}$ is isomorphic to the functor which assigns to the object $n^{+}$ of $\Gamma^{\text {op }}$ the $B$-module $B[x]^{\otimes_{B} n}$. By a theorem of Pirashvili and Richter [36, Thm. 1], the homotopy groups $\pi_{*} D B$ are thus isomorphic to the $\Gamma$-homology $H \Gamma_{*}(B[x] \mid B ; B)$ of the polynomial algebra $B[x]$ relative to $B$. The second isomorphism in (3.4) is due to Basterra and McCarthy [2, who show that for Eilenberg-MacLane spectra of classical rings, topological André-Quillen homology coincides with $\Gamma$-homology. The survey article [3] by Basterra and Richter discusses all these identifications in more detail.

The isomorphisms (3.4) do not mention the multiplicative structure of $\pi_{*} D B$. In Sections 5.1 and 7.9 of [46] we associate to any augmented commutative $B$-algebra $A$ a $D B$-module $\Sigma_{B}^{\infty} A$ which models the suspension spectrum of $A$ as an augmented commutative $B$-algebra. The underlying $\Gamma$-space of $\Sigma_{B}^{\infty} A$ sends $n^{+} \in \Gamma^{\mathrm{op}}$ to the $B$-module $A^{\otimes_{B} n}$. So [36, Thm. 1] yields an isomorphism of graded abelian groups

$$
\pi_{*}\left(\Sigma_{B}^{\infty} A\right) \cong H \Gamma_{*}(A \mid B ; B)
$$

(at least if $A$ is flat as a $B$-module). The $D B$-action on $\Sigma_{B}^{\infty} A$ gives more structure to $\Gamma$-homology, since the left hand side above has a natural action of the graded ring $\pi_{*} D B \cong H \Gamma_{*}(B[x] \mid B ; B)$. We see moreover that the homotopical object underlying $\Gamma$-homology is not just a chain complex, but a $D B$-module spectrum.

The main objective of this paper is the study of the homotopy type of $D B$ as a ring spectrum and of a close relationship to formal group theory. More precisely we will describe the space of Gamma-ring maps from $H \mathbb{Z}$, the EilenbergMacLane Gamma-ring of the integers (2.8), to $D B$ in terms of formal group law data. Unless stated otherwise, formal group laws will always be 1-dimensional and commutative. To see how non-commutative and higher-dimensional formal group laws fit into our context see Remarks 13.4 and 13.5. 
Construction 3.5 Suppose that $F$ is a 1-dimensional and commutative formal group law over the commutative ring $B$. In other words, $F$ is a power series in two variables $x$ and $y$ with coefficients in $B$ which satisfies

$$
\begin{aligned}
F(x, 0) & =x=F(0, x), \\
F(x, y) & =F(y, x) \text { and } \\
F(F(x, y), z) & =F(x, F(y, z)) .
\end{aligned}
$$

We define a map $F_{*}: H \mathbb{Z} \longrightarrow D B$ of Gamma-rings. For every pointed set $K$ we have to specify a map

$$
F_{*}(K): \widetilde{\mathbb{Z}}[K]=H \mathbb{Z}(K) \longrightarrow D B(K) \subseteq \widetilde{B} \llbracket K \rrbracket
$$

which is natural in $K$ and respects the multiplication and unit maps. The map $F_{*}(K)$ simply takes a sum $\sum_{k \in K} a_{k} \cdot k$ of generators of the free abelian group on $K$ to the formal sum

$$
\sum_{k \in K}^{F}\left[a_{k}\right]_{F}(k)
$$

with respect to $F$, of the same elements viewed as generators of the power series ring. Here $[n]_{F}$ denotes the $n$-series of the formal group law $F$ for every integer $n \in \mathbb{Z}$. We omit the verification that the map $F_{*}$ indeed commutes with the multiplication and unit map. For example, on the level of underlying monoids this means that the map

$$
[-]_{F}: \mathbb{Z} \cong H \mathbb{Z}\left(1^{+}\right) \longrightarrow D B\left(1^{+}\right)=x \cdot B \llbracket x \rrbracket
$$

is a homomorphism from the multiplicative monoid of the integers to the monoid of power series without constant term under substitution, ie, it boils down to the relation

$$
[n]_{F}\left([m]_{F}(x)\right)=[n \cdot m]_{F}(x)
$$

for $n, m \in \mathbb{Z}$

Remark 3.6 We offer two additional ways of looking at the above construction of the Gamma-ring map $F_{*}$; the three definitions correspond to looking at a commutative 1-dimensional formal group law as either

(a) a power series $F(x, y)$ in two variables with certain properties,

(b) an abelian cogroup structure, in the category of complete, augmented commutative $B$-algebras, on the power series ring $B \llbracket x \rrbracket$,

(c) or a morphism of algebraic theories from the theory of abelian groups to the theory of complete, augmented, commutative $B$-algebras. 
The first point of view leads to the explicit formula for the Gamma-ring map $F_{*}$ that was just given in Construction 3.5 .

The interpretation (b) of a formal group law exhibits the Gamma-ring map $F_{*}$ as a special case of a more general construction associated to an abelian cogroup object. Indeed, to every object $X$ in a pointed category with coproducts $\mathcal{C}$ one can associate an endomorphism Gamma-ring $\operatorname{End}_{\mathcal{C}}(X)$ (see [46, 4.6] or 13.3). Then every abelian cogroup structure on the object $X$ leads to a map of Gamma-rings $H \mathbb{Z} \longrightarrow \operatorname{End}_{\mathcal{C}}(X)$; we refer to 13.3 for more details.

Perspective (c) leads to a compact description in the language of algebraic theories [6, 3.3.1]. Specifying a 1-dimensional, commutative formal group law over $B$ is the same as specifying a morphism of algebraic theories [6, 3.7.1] from the theory of abelian groups to the theory of complete, augmented, commutative $B$-algebras. The construction [44, 4.5] which associates to a pointed algebraic theory $T$ its stable Gamma-ring $T^{s}$ is functorial for morphisms of algebraic theories. Now $H \mathbb{Z}$ is the Gamma-ring associated to the theory of abelian groups and $D B$ is the Gamma-ring associated to the theory of complete, augmented, commutative $B$-algebras. Hence a formal group law $F$ defines a morphism of algebraic theories, thus a morphisms of associated Gamma-rings.

Example 3.7 The additive formal group law is given by

$$
F^{a}(x, y)=x+y .
$$

The associated Gamma-ring map $F_{*}^{a}$ is the composite

$$
H \mathbb{Z} \longrightarrow H B \stackrel{\text { incl. }}{\longrightarrow} D B
$$

of the unique Gamma-ring map $H \mathbb{Z} \longrightarrow H B$ with the 'inclusion' of $H B$ into $D B$ as the linear power series. Conversely, this is the only case in which the Gamma-ring map $F_{*}$ factors over the inclusion $H B \longrightarrow D B$ on the point-set level: the power series $F(x, y)$ can be recovered from the Gamma-ring map $F_{*}$ as the image of $x+y \in \mathbb{Z}[x, y]$ under

$$
F_{*}: \mathbb{Z}[x, y] \cong H \mathbb{Z}\left(\{x, y\}^{+}\right) \longrightarrow D B\left(\{x, y\}^{+}\right) \subseteq B \llbracket x, y \rrbracket ;
$$

so if $F_{*}$ factors over $H B$, then $F(x, y)$ has only linear terms, so that necessarily $F(x, y)=x+y$.

Another formal group law which exists over any $\operatorname{ring} B$ is the multiplicative one given by

$$
F^{m}(x, y)=x+y+x y
$$


The multiplicative formal group law can be used to express the Gamma-ring $D B$ additively as the smash product of the Eilenberg-MacLane Gamma-rings for $B$ and $\mathbb{Z}$; indeed the composite map

$$
H B \wedge^{L} H \mathbb{Z} \stackrel{\text { incl. } \wedge^{L} F_{*}^{m}}{\longrightarrow} D B \wedge^{L} D B \stackrel{\mu}{\longrightarrow} D B
$$

is in the same homotopy class as the stable equivalence of Theorem 3.2 (b).

The homotopical significance of the point-set level construction of 3.5 is summarized in the following

Theorem 3.8 Construction 3.5 which to a 1-dimensional, commutative formal group law $F$ associates the Gamma-ring map $F_{*}: H \mathbb{Z} \longrightarrow D B$ induces a natural bijection

$$
\mathrm{FGL}(B) / \text { strict isomorphism } \stackrel{\cong}{\longrightarrow}[H \mathbb{Z}, D B]_{\text {Gamma-rings }}
$$

between the strict isomorphism classes of formal group laws over $B$ and the set of maps from $H \mathbb{Z}$ to $D B$ in the homotopy category of Gamma-rings.

Theorem 3.8 is the $\pi_{0}$-part of a space level statement relating formal groups to Gamma-ring maps between $H \mathbb{Z}$ and $D B$ in Theorem 5.2. In the simplicial model category of Gamma-rings every pair of objects has a homomorphism space (ie, simplicial set). As usual with model categories, in order to give the morphism space a homotopy invariant meaning, the source object has to be replaced by a weakly equivalent cofibrant one, and the target object has to be replaced by a weakly equivalent fibrant one. Then the components of the (derived) space of Gamma-ring maps are the morphisms in the homotopy category of Gamma-rings. Theorem 5.2 below identifies the derived homomorphism space of Gamma-ring maps from $H \mathbb{Z}$ to $D B$. The answer is given in terms of formal group law data and the simplicial monoid of homotopy units of $D B$.

3.9 Homotopy units Let $R$ be a Gamma-ring and $R^{\mathrm{f}}$ a stably fibrant replacement of $R$ in the model category structure of [44, 2.5]. As for any Gammaring, the underlying space $R^{\mathrm{f}}\left(1^{+}\right)$is a simplicial monoid with product induced by the multiplication map $R^{\mathrm{f}} \wedge R^{\mathrm{f}} \longrightarrow R^{\mathrm{f}}$. Moreover, $R^{\mathrm{f}}\left(1^{+}\right)$is a model for the infinite loop space of the spectrum represented by $R$. We define the homotopy units $R^{\times}$as the union of the invertible components of the simplicial monoid $R^{\mathrm{f}}\left(1^{+}\right)$. So $R^{\times}$is a group-like simplicial monoid which is independent up to weak equivalence of the choice of fibrant replacement. Moreover there are natural isomorphisms of homotopy groups

$$
\pi_{0} R^{\times} \cong \operatorname{units}\left(\pi_{0} R\right) \text { and } \pi_{i} R^{\times} \cong \pi_{i} R \text { for } i \geq 1 .
$$


For any classical ring $A$, the units of $A$ act by conjugation on $A$ and hence on the set of ring homomorphisms from any other ring to $A$. In Section 4 we make sense of the analogous conjugation action for Gamma-rings. For any Gammaring $R$ we construct, after change of models, a strict action of the homotopy units $R^{\times}$on $R$ by Gamma-ring homomorphisms. More precisely we introduce a simplicial group $U R^{\times}$, weakly equivalent to $R^{\times}$, and a fibrant Gamma-ring, stably equivalent to $R$, and on which $U R^{\times}$acts by conjugation. Below we only use the action of the simplicial subgroup $U R_{1}^{\times}$, the connected component of the unit element. We can consider the homotopy orbit space of the conjugation action of $U R_{1}^{\times}$on the simplicial set $\operatorname{Ring}(H \mathbb{Z}, R)=\operatorname{map}_{\mathcal{G} R}\left(H \mathbb{Z}^{\mathrm{c}}, R^{\mathrm{f}}\right)$. We denote that homotopy orbit space by $\operatorname{Ring}(H \mathbb{Z}, R) /$ conj.

Construction 3.5 associates to every formal group law over $B$ a Gamma-ring map from the Eilenberg-MacLane Gamma-ring $H \mathbb{Z}$ to $D B$. This map gives rise to a point in the space of Gamma-ring maps $\operatorname{Ring}(H \mathbb{Z}, D B)$. In Section 5 we extend this to a natural weak map from the classifying space $\mathcal{F} \mathcal{G} \mathcal{L}^{\operatorname{str}}(B)$ of the groupoid of formal group laws and strict isomorphisms to the homotopy orbits space of the conjugation action, ie, we construct a diagram of simplicial sets

$$
\mathcal{F} \mathcal{G} \mathcal{L}^{\operatorname{str}}(B) \stackrel{\sim}{\longleftarrow} \widetilde{\mathcal{F G L}}^{\text {str }}(B) \stackrel{\kappa}{\longrightarrow} \operatorname{Ring}(H \mathbb{Z}, D B) / \text { conj. }
$$

in which the first map is a weak equivalence. The main result of this paper, Theorem 5.2. says that the map $\kappa: \widetilde{\mathcal{F G \mathcal { L }}}^{\text {str }}(B) \longrightarrow \operatorname{Ring}(H \mathbb{Z}, D B) /$ conj. is a weak equivalence. Theorem 3.8 is the bijection induced on path components by the weak equivalence $\kappa$.

\section{The conjugation action}

In this section we construct the conjugation action of the homotopy units of a Gamma-ring on the Gamma-ring. In view of the application to $D B$ we need a construction relative to a group which maps to the multiplicative monoid of the Gamma-ring. In the example of $D B$ that group is the group of invertible power series on one generator over $B$.

Construction 4.1 We consider a Gamma-ring $R$ together with a simplicial group $G$ and a homomorphism of simplicial monoids $\psi: G \longrightarrow R\left(1^{+}\right)$. The conjugation action of $G$ on $R$ is described by a map of simplicial monoids

$$
c: G \longrightarrow \operatorname{map}_{\mathcal{G R}}(R, R) \text {. }
$$


Here for $g \in G$ the conjugation map $c(g): R \longrightarrow R$ is defined at a pointed set $K$ as the composite

$$
\begin{aligned}
R(K) \stackrel{g \wedge \mathrm{id} \wedge g^{-1}}{\longrightarrow} G_{+} \wedge R(K) \wedge G_{+} \stackrel{\psi \wedge \mathrm{id} \wedge \psi}{\stackrel{\text { mult. }}{\longrightarrow}} R\left(1^{+}\right) \wedge R(K) \wedge R\left(1^{+} \wedge K \wedge 1^{+}\right) \cong R(K) .
\end{aligned}
$$

We omit the formal verification that the conjugation map is in fact a homomorphism of Gamma-rings, that the definition extends to higher dimensional simplices of $G$, and that the formula $c\left(g \cdot g^{\prime}\right)=c(g) \circ c\left(g^{\prime}\right)$ holds. The monoid map $c$ can now be used to let the group $G$ act on the space of Gamma-ring maps from any Gamma-ring $S$ to $R$ via

$$
G \times \operatorname{map}_{\mathcal{G R}}(S, R) \longrightarrow \operatorname{map}_{\mathcal{G R}}(S, R), \quad(g, f) \mapsto c(g) \circ f .
$$

The goal of this section is to extend the conjugation action from the given group $G$ to the homotopy units (3.9) of $R$. The problem is that Construction 4.1 makes use of strict inverses, whereas the homotopy units $R^{\times}$are only a group-like simplicial monoid. One way to solve this would be to find a stable equivalence of Gamma-rings from $R$ to some stably fibrant Gamma-ring $R^{\mathrm{f}}$ which has the property that every element in an invertible component of $R^{\mathrm{f}}\left(1^{+}\right)$has a point-set inverse. But it seems unlikely that this can be done in general, and we use a different approach.

Construction 4.2 As above we consider a Gamma-ring $R$ together with a simplicial group $G$ and a homomorphism of simplicial monoids $\psi: G \longrightarrow$ $R\left(1^{+}\right)$. If $R \longrightarrow R^{\mathrm{f}}$ is a stably fibrant replacement of $R$ in the model category of Gamma-rings then $R^{\times}$was defined in 3.9 as the simplicial monoid of invertible components in $R^{\mathrm{f}}\left(1^{+}\right)$. The image of the simplicial group $G$ under the map $R\left(1^{+}\right) \longrightarrow R^{\mathrm{f}}\left(1^{+}\right)$is contained in the invertible components, which provides a morphism of simplicial monoids $G \longrightarrow R^{\times}$. We explained in 4.1 how the group $G$ acts on spaces of Gamma-ring maps into $R$, and we now want to extend this to a conjugation action of the homotopy units $R^{\times}$.

We start by factoring the homomorphism $G \longrightarrow R^{\times}$in the model category of simplicial monoids (a special case of [39, II.4 Thm. 4]) as a cofibration followed by an acyclic fibration

$$
G \longrightarrow c R^{\times} \stackrel{\sim}{\longrightarrow} R^{\times} .
$$

We denote by $U R^{\times}$the algebraic group completion of the simplicial monoid $c R^{\times}$. So $U R^{\times}$is obtained from $c R^{\times}$by formally adjoining inverses in every simplicial dimension. Since the map $G \longrightarrow c R^{\times}$is a cofibration of simplicial 
monoids, the monoid $c R^{\times}$is a retract of a simplicial monoid which is dimensionwise a free product of a group and a free monoid [39, II p. 4.11 Rem. 4]. By the following Lemma the group completion map $c R^{\times} \longrightarrow U R^{\times}$is thus a weak equivalence.

Lemma 4.3 Let $M$ be a simplicial monoid which in every dimension is a free product of a group and a free monoid, and such that $\pi_{0} M$ is a group. Then the group completion map $M \longrightarrow U M$ is a weak equivalence.

Proof We call a simplicial monoid $N$ good if the group completion map $N \longrightarrow$ $U N$ induces a weak equivalence $B N \longrightarrow B U N$ of classifying spaces. For any simplicial monoid $N$ whose components form a group, $B N$ is a delooping of $N$, ie, the map $|N| \longrightarrow \Omega|B N|$ is a weak equivalence; this follows for example by applying [8, B.4] to the sequence of bisimplicial sets $N \longrightarrow E_{\bullet} N \longrightarrow B_{\bullet} N$. So it suffices to show that any monoid as in the statement of the lemma is good.

Suppose $M$ and $N$ are good, discrete monoids. We claim that then the free product $M * N$ is also good. By [33, Lemma 4], the canonical map of simplicial sets $B M \vee B N \longrightarrow B(M * N)$ is a weak equivalence. Since $U(M * N) \cong$ $U M * U N$ (the coproduct in the category of groups coincides with the free product of underlying monoids), the map $B U M \vee B U N \longrightarrow B U(M * N)$ is also a weak equivalence and the claim follows.

Every group, viewed as a constant simplicial monoid, is good. The classifying spaces of the free monoid on one generator and of the free group on one generator are both weakly equivalent to a circle. So a free monoid on one generator is good. By the above and by direct limit, a free product of a group with a free monoid is good.

The classifying space $B N$ of a simplicial monoid $N$ is the diagonal of the bisimplicial set given by the classifying spaces $B N_{m}$ of the individual monoids $N_{m}$ in the various simplicial dimensions. Hence if $N$ is a simplicial monoid such that the discrete monoid $N_{m}$ is good for all $m \geq 0$, then $N$ itself is good. This proves the lemma.

We take the adjoint $\mathbb{S}\left[c R^{\times}\right] \longrightarrow R^{\mathrm{f}}$ of the monoid map $c R^{\times} \longrightarrow R^{\times} \longrightarrow R^{\mathrm{f}}\left(1^{+}\right)$ (where $\mathbb{S}\left[c R^{\times}\right]$is the monoid Gamma-ring (2.8)) and factor it in the model category of Gamma-rings as a cofibration followed by an acyclic fibration

$$
\mathbb{S}\left[c R^{\times}\right] \longrightarrow R_{1} \stackrel{\sim}{\longrightarrow} R^{\mathrm{f}} .
$$


We then define another Gamma-ring $R_{2}$ as the pushout, in the category of Gamma-rings, of the diagram

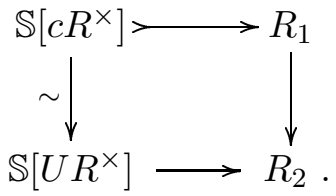

For every pointed simplicial set $K$ the $\Gamma$-space $\mathbb{S} \wedge K$ is cofibrant; in particular the underlying $\Gamma$-spaces of $\mathbb{S}\left[c R^{\times}\right]$and $\mathbb{S}\left[U R^{\times}\right]$are cofibrant. By [47, 4.1 (3)] the underlying $\Gamma$-space of $R_{1}$ is also cofibrant, so by the following lemma the map $R_{1} \longrightarrow R_{2}$ is a stable equivalence of Gamma-rings.

Lemma 4.4 Consider a diagram of Gamma-rings

$$
X \stackrel{\sim}{\longleftarrow} Y \longrightarrow
$$

in which the left map is a stable equivalence, the right map is a cofibration and all three Gamma-rings are cofibrant as $\Gamma$-spaces. Then the map from $Z$ to the pushout of the diagram is also a stable equivalence.

Proof We denote the pushout of the diagram by $P$. We first consider the situation where the map $Y \longrightarrow Z$ is obtained by cobase change from a generating cofibration. In other words we assume that there exists a cofibration $K \longrightarrow L$ of $\Gamma$-spaces such that $Z$ is the pushout of the diagram of Gamma-rings

$$
Y \longleftarrow T(K) \longrightarrow T(L)
$$

where $T$ denotes the tensor algebra functor. This special kind of pushout in the category of Gamma-rings is analyzed in the proof of [47, Lemma 6.2]. The pushout $Z$ is then the colimit of a sequence of cofibrations of $\Gamma$-spaces

$$
Y=Z_{0} \longrightarrow Z_{1} \longrightarrow \cdots \longrightarrow Z_{n} \longrightarrow \cdots
$$

for which the subquotient $Z_{n} / Z_{n-1}$ is isomorphic to $(L / K)^{\wedge n} \wedge Y^{\wedge(n+1)}$. In the same way the composite pushout $P$ is the colimit of a sequence of cofibrations of $\Gamma$-spaces with subquotients isomorphic to $(L / K)^{\wedge n} \wedge X^{\wedge(n+1)}$. The smash product of $\Gamma$-spaces preserves stable equivalences between cofibrant objects [29. Thm. 5.12], so the induced maps on the subquotients of the filtrations for $Z$ and $P$ are all stable equivalences. So the maps induced on all finite stages, and finally the map $Z \longrightarrow P$ on colimits are also stable equivalences.

By induction the lemma thus holds whenever the cofibration $Y \longrightarrow Z$ is the composite of finitely many maps obtained by cobase changes from generating 
cofibrations. Since homotopy groups of $\Gamma$-spaces commute with transfinite compositions over cofibrations, the lemma holds whenever $Y \longrightarrow Z$ is such a transfinite composition of cobase changes of generating cofibrations. Finally, if the lemma holds for a cofibration $Y \longrightarrow Z$, then it also holds for any retract. But all cofibrations of Gamma-rings are obtained by a sequence of these constructions by the small object argument.

We finally let $R_{3}$ be a stably fibrant replacement of the Gamma-ring $R_{2}$. We can display all the relevant objects thus constructed in a commutative diagram of Gamma-rings

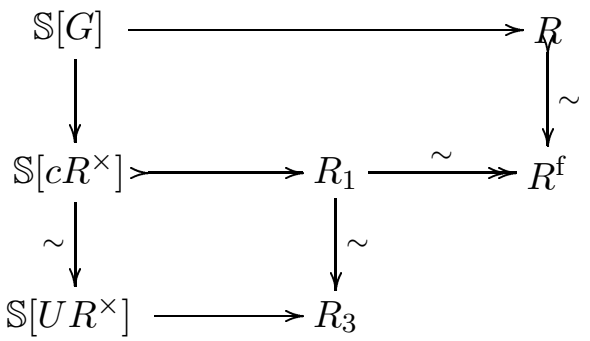

In this diagram the Gamma-rings $R^{\mathrm{f}}, R_{1}$ and $R_{3}$ are fibrant, and the maps between them are stable equivalences. Furthermore, the induced map from the simplicial group $U R^{\times}$to the invertible components of the underlying monoid of $R_{3}$ is a weak equivalence. As described in 4.1 the simplicial group $U R^{\times}$acts by conjugation on $R_{3}$ via homomorphisms of Gamma-rings, and this action extends the action of $G$.

Remark 4.5 Construction 4.2 can be made functorial in the triple $(R, G, \psi$ : $G \longrightarrow R\left(1^{+}\right)$) since the factorizations in the model categories of simplicial monoids and of Gamma-rings can be made functorial.

\section{The comparison map}

We now use the conjugation action of the previous section in the case of the Gamma-ring $D B$. We obtain a model for the homotopy invariant space of Gamma-ring maps from $H \mathbb{Z}$ to $D B$ on which the homotopy units of $D B$ act by conjugation. So we can form the homotopy orbit space with respect to the conjugation action. We then construct a weak map from the classifying space of the groupoid of formal group laws and strict isomorphisms to the homotopy orbit space of the conjugation action. In the remaining sections we show that that map is a weak equivalence. 
In the later sections we need a version of the weak map from the classifying space $\mathcal{F} \mathcal{G}^{\operatorname{str}}(B)$ to the homotopy orbit space for other Gamma-rings. So we set up the construction of the (weak) map in a slightly more general context.

Construction 5.1 Again we consider a Gamma-ring $R$, a simplicial group $G$ and a homomorphism of simplicial monoids $\psi: G \longrightarrow R\left(1^{+}\right)$. We now make the additional assumption that the image of $G$ lands in the unit component of $R$, ie, the composite map

$$
G \stackrel{\psi}{\longrightarrow} R\left(1^{+}\right) \longrightarrow \pi_{0} R
$$

is constant with value $1 \in \pi_{0} R$. This assumption is not very important, but it slightly simplifies certain arguments later. Moreover we are given a simplicial set $X$ with an action of the simplicial group $G$ and suppose we are are also given a $G$-equivariant map $X \longrightarrow \operatorname{map}_{\mathcal{G R}}(H \mathbb{Z}, R)$, where $G$ acts on the mapping space by conjugation (4.1). In our main example, $R$ will be the Gamma-ring $D B$ and $G$ will be the discrete group of power series in one variable with leading term $x$ and with multiplication give by substitution. Furthermore, $X$ will be the set of formal group laws over $B$, and the map $\mathrm{FGL}(B) \longrightarrow \operatorname{map}_{\mathcal{G R}}(H \mathbb{Z}, D B)$ takes $F$ to $F_{*}$.

In Construction 4.2 we produced a simplicial group $U R^{\times}$, a homomorphism of simplicial groups $G \longrightarrow U R^{\times}$and a commutative diagram of Gamma-rings

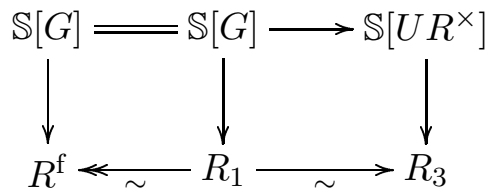

in which the lower horizontal maps are stable equivalences between stably fibrant models of $R$. Furthermore, the induced map from the simplicial group $U R^{\times}$to the invertible components of the underlying monoid of $R_{3}$ is a weak equivalence. The simplicial group $G$ acts by conjugation on $R, R_{1}$ and $R_{3}$, hence on the spaces of Gamma-ring maps from any other Gamma-ring into $R$, $R_{1}$ and $R_{3}$. If $S$ is a cofibrant Gamma-ring, then the lower horizontal maps induce weak equivalences

$$
\operatorname{map}_{\mathcal{G} R}\left(S, R^{\mathrm{f}}\right) \stackrel{\sim}{\longleftarrow} \operatorname{map}_{\mathcal{G} R}\left(S, R_{1}\right) \stackrel{\sim}{\longrightarrow} \operatorname{map}_{\mathcal{G} R}\left(S, R_{3}\right)
$$

which are $G$-equivariant. Furthermore the action of $G$ on $\operatorname{map}_{\mathcal{G} R}\left(S, R_{3}\right)$ extends to an action of the group $U R^{\times}$. So we have extended, up to weak equivalence, the action of the group $G$ to the action of a simplicial group weakly equivalent to the homotopy units of $R$. 
If we now choose a cofibrant replacement $H \mathbb{Z}^{\mathrm{c}} \longrightarrow H \mathbb{Z}$ in the model category of Gamma-rings, then the space $\operatorname{map}_{\mathcal{G R}}\left(H \mathbb{Z}^{\mathrm{c}}, R_{3}\right)$ is a model for the homotopy invariant space of Gamma-ring maps. Furthermore, the simplicial group $U R^{\times}$acts on this space by conjugation, hence so does its subgroup $U R_{1}^{\times}$, the connected component of the identity element. We abbreviate the space $\operatorname{map}_{\mathcal{G R}}\left(H \mathbb{Z}^{\mathrm{c}}, R_{3}\right)$ to $\operatorname{Ring}(H \mathbb{Z}, R)$ and write $\operatorname{Ring}(H \mathbb{Z}, R) /$ conj. for the homotopy orbit space of the conjugation action of the connected simplicial group $U R_{1}^{\times}$

$$
\operatorname{Ring}(H \mathbb{Z}, R) / \text { conj. }=\operatorname{map}_{\mathcal{G R}}\left(H \mathbb{Z}^{\mathrm{c}}, R_{3}\right)_{h U R_{1}^{\times}} .
$$

We now use the $G$-space $X$ and the equivariant map $X \longrightarrow \operatorname{map}_{\mathcal{G R}}(H \mathbb{Z}, R)$ to construct a weak map from the homotopy orbit space $X_{h G}$ to the homotopy orbit space $\operatorname{Ring}(H \mathbb{Z}, R) /$ conj.. We let $\widetilde{X}$ denote the pullback of the diagram



Since all spaces in the diagram have an action by the group $G$ and all maps are equivariant, the group $G$ acts on the space $\widetilde{X}$. Since the map $R_{1} \longrightarrow R^{\mathrm{f}}$ is an acyclic fibration of Gamma-rings, the induced map on homomorphism spaces is an acyclic fibration, hence so is the map $\widetilde{X} \longrightarrow X$. The stable equivalence of Gamma-rings $R_{1} \longrightarrow R_{3}$ induces a $G$-equivariant map of homomorphism $\operatorname{spaces} \operatorname{map}_{\mathcal{G R}}\left(H \mathbb{Z}^{\mathrm{c}}, R_{1}\right) \longrightarrow \operatorname{map}_{\mathcal{G R}}\left(H \mathbb{Z}^{\mathrm{c}}, R_{3}\right)$.

The conjugation action of $G$ on the target space extends to an action of the simplicial group $U R^{\times}$. By our assumption on the homomorphism $\psi: G \longrightarrow$ $R\left(1^{+}\right)$the image of $G$ lands in the identity component $U R_{1}^{\times}$. We denote by $\kappa$ the map induced on homotopy orbit spaces

$$
\begin{aligned}
& \kappa: \tilde{X}_{h G} \longrightarrow \operatorname{map}_{\mathcal{G R}}\left(H \mathbb{Z}^{\mathrm{c}}, R_{1}\right)_{h G} \\
& \longrightarrow \operatorname{map}_{\mathcal{G R}}\left(H \mathbb{Z}^{\mathrm{c}}, R_{3}\right)_{h U R_{1}^{\times}}=\operatorname{Ring}(H \mathbb{Z}, R) / \text { conj. }
\end{aligned}
$$

So altogether we have obtained a weak map of homotopy orbit spaces

$$
X_{h G} \stackrel{\sim}{\longleftarrow} \tilde{X}_{h G} \stackrel{\kappa}{\longrightarrow} \operatorname{Ring}(H \mathbb{Z}, R) / \text { conj. }
$$

Now we return to the main example and apply Construction 5.1 to the Gammaring $D B$. In this case $G$ is the discrete group $\Phi(B)$ of power series $\varphi(x)$ in one variable over $B$ with leading term $x$, with composition (substitution) of power series as the group structure. This group acts on the set of formal group laws over $B$ via

$$
F^{\varphi}(x, y)=\varphi\left(F\left(\varphi^{-1}(x), \varphi^{-1}(y)\right)\right) .
$$


In fact, $F^{\varphi}$ is defined so that $\varphi: F \longrightarrow F^{\varphi}$ is a strict isomorphism of formal group laws. The homomorphism $\Phi(B) \longrightarrow D B\left(1^{+}\right) \cong x \cdot B \llbracket x \rrbracket$ is the inclusion. Because of the equality

$$
\left(F^{\varphi}\right)_{*}=\varphi \cdot F_{*} \cdot \varphi^{-1}
$$

as maps of Gamma-rings $H \mathbb{Z} \longrightarrow D B$, the assignment $F \mapsto F_{*}$ from the set FGL $(B)$ of formal group laws to the set of Gamma-ring maps from $H \mathbb{Z}$ to $D B$ is $\Phi(B)$-equivariant. The homotopy orbit space $\mathrm{FGL}(B)_{h \Phi(B)}$ is isomorphic to the classifying space of the groupoid of formal group laws and strict isomorphisms, which we denote by $\mathcal{F} \mathcal{G L}^{\text {str }}(B)$. So Construction 5.1 yields maps

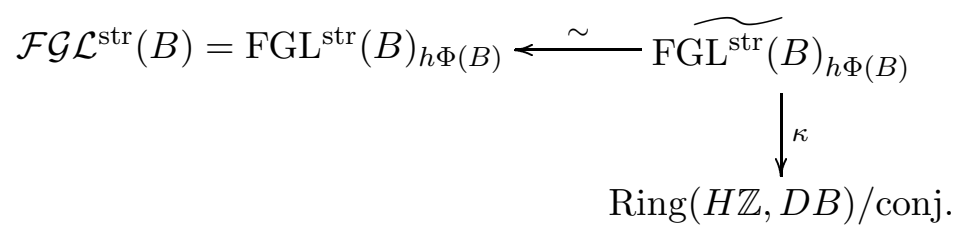

where the upper map is a weak equivalence. We use the notation $\widetilde{\mathcal{F G L}}^{\text {str }}(B)$ for the homotopy orbit space $\widetilde{\mathrm{FGL}^{\mathrm{str}}}(B)_{h \Phi(B)}$. The following theorem is our main result:

Theorem 5.2 The map

$$
\kappa: \widetilde{\mathcal{F G \mathcal { L }}}^{\mathrm{str}}(B) \longrightarrow \operatorname{Ring}(H \mathbb{Z}, D B) / \text { conj. }
$$

is a weak equivalence.

The proof of Theorem 5.2 occupies the rest of this paper. Since the homotopy orbit space construction defining $\operatorname{Ring}(H \mathbb{Z}, D B) /$ conj. involves a connected simplicial group, the quotient map

$$
\operatorname{Ring}(H \mathbb{Z}, D B) \longrightarrow \operatorname{Ring}(H \mathbb{Z}, D B) / \text { conj. }
$$

induces a bijection of path components. The set $[H \mathbb{Z}, D B]_{\text {Gamma-rings }}$ is canonically isomorphic to the components of the mapping space $\operatorname{Ring}(H \mathbb{Z}, D B)$, so Theorem 3.8 is just the bijection of path components induced by the weak equivalence $\kappa$ of Theorem 5.2 .

In addition to the homotopy classes of Gamma-ring maps, Theorem 5.2 allows us to identify the higher homotopy groups of the space $\operatorname{Ring}(H \mathbb{Z}, D B)$ of Gammaring maps. Since the group $\pi_{1}(D B)^{\times} \cong \pi_{1} D B$ is trivial by 3.2 (b), the simplicial group $(D B)_{1}^{\times}$is 1 -connected, so the quotient map $\operatorname{Ring}(H \mathbb{Z}, D B) \longrightarrow$ $\operatorname{Ring}(H \mathbb{Z}, D B) /$ conj. induces an equivalence of fundamental groupoids. Together with Theorem 5.2 this implies that the fundamental groupoid of the 
space $\operatorname{Ring}(H \mathbb{Z}, D B)$ is equivalent to the groupoid $\mathcal{F} \mathcal{G} \mathcal{L}^{\text {str }}(B)$. In particular this yields isomorphisms

$$
\pi_{1} \operatorname{Ring}\left(H \mathbb{Z}, D B ; F_{*}\right) \cong \operatorname{Aut}^{\text {strict }}(F)
$$

between the fundamental group at the basepoint $F_{*} \in \operatorname{Ring}(H \mathbb{Z}, D B)$ and the strict automorphism group of $F$. Since the homotopy orbit space of the conjugation action is weakly equivalent to the classifying space of a groupoid, its homotopy groups are trivial above dimension 1 ; so for every $F$ the action map

$$
U(D B)_{1}^{\times} \longrightarrow \operatorname{Ring}(H \mathbb{Z}, D B), \quad u \longmapsto u \cdot F_{*} \cdot u^{-1}
$$

induces isomorphisms of homotopy groups $\pi_{n} D B \cong \pi_{n} \operatorname{Ring}\left(H \mathbb{Z}, D B ; F_{*}\right)$ for $n \geq 2$.

Example 5.3 For algebras over the rational numbers, the map $\kappa$ of Theorem 5.2 is trivially an equivalence. Indeed, if $B$ is a $\mathbb{Q}$-algebra, and $F$ a 1-dimensional and commutative formal group law over $B$, then there is a strict isomorphism (called the logarithm of $F$ ) between $F$ and the additive formal group law [19, III.1 Cor. 1]. Moreover, $F$ has no non-trivial strict automorphisms, so the classifying space of the groupoid $\mathcal{F} \mathcal{G} \mathcal{L}^{\operatorname{str}}(B)$ is weakly contractible.

On the other hand, the Gamma-ring $D B$ is now stably equivalent to the Eilenberg-MacLane Gamma-ring $H B$ by Theorem 3.2 (b). So both the space $\operatorname{Ring}(H \mathbb{Z}, D B)$ and the unit component of $(D B)^{\times}$are weakly contractible, hence so is the homotopy orbit space $\operatorname{Ring}(H \mathbb{Z}, D B) /$ conj.

Remark 5.4 Instead of taking homotopy orbits with respect to the connected simplicial group $U(D B)_{1}^{\times}$one can divide out the conjugation action of the entire homotopy units $U(D B)^{\times}$on the space $\operatorname{Ring}(H \mathbb{Z}, D B)$. The resulting orbit space receives a (weak) map from the groupoid of formal group laws and all (ie, not necessarily strict) isomorphisms. The same proof as for Theorem 5.2 shows that that map

$$
\widetilde{\mathcal{F} \mathcal{G L}}(B) \longrightarrow \operatorname{Ring}(H \mathbb{Z}, D B)_{h U(D B)^{\times}}
$$

is a weak equivalence.

\section{A filtration of $D B$}

The Gamma-ring $D B$ has a natural filtration arising from powers of the augmentation ideal of the power series rings. There are truncated versions $D_{k} B$ of 
the Gamma-ring $D B$ and analogues of the map $\kappa$ of Theorem 5.2 for every $k$. In this section we reduce the proof of Theorem 5.2 to the analogous statement about the stages of the filtration, see Theorem 6.4

For $k \geq 1$ we denote by $D_{k} B$ the truncated version of the Gamma-ring $D B$ obtained by dividing out all power series in the $(k+1)$-st power of the augmentation ideal. So as a $\Gamma$-space,

$$
D_{k} B(K)=\operatorname{kernel}\left(\widetilde{B} \llbracket K \rrbracket / I^{k+1} \longrightarrow \widetilde{B} \llbracket * \rrbracket=B\right)
$$

where $I=K \cdot \widetilde{B} \llbracket K \rrbracket$ is the ideal of $\widetilde{B} \llbracket K \rrbracket$ consisting of power series without constant term. The unit map again comes from the inclusion of generators and the multiplication is induced by substitution of (truncated) power series, similar to the definition for $D B$. In other words: $D_{k} B$ has a unique Gammaring structure for which the natural projection map $D B \longrightarrow D_{k} B$ is a homomorphism of Gamma-rings. Note that $D_{1} B$ is isomorphic to the EilenbergMacLane Gamma-ring $H B$. There are maps of Gamma-rings $D B \longrightarrow D_{k} B$ and $D_{k} B \longrightarrow D_{k-1} B$ induced by truncation of power series.

Now we apply Construction 5.1 to the Gamma-ring $D_{k} B$. The group we work relative to is $\Phi_{k}(B)$, the quotient of the group $\Phi(B)$ of power series over $B$ with leading term $x$ by the normal subgroup of power series which are congruent to the power series $x$ modulo $x^{k+1}$. The group $\Phi_{k}(B)$ injects into the monoid $D_{k} B\left(1^{+}\right)$, so Construction 4.2 provides a diagram of Gamma-rings

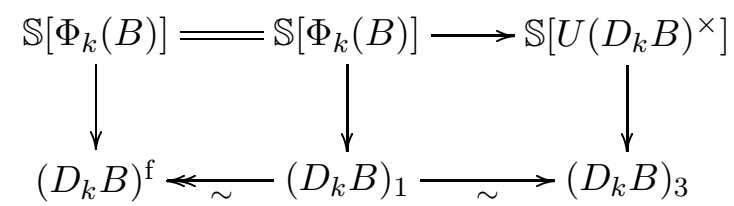

in which the lower horizontal maps are stable equivalences between fibrant Gamma-rings. Furthermore the induced map from the simplicial group $U\left(D_{k} B\right)^{\times}$to the invertible components of the underlying monoid of $\left(D_{k} B\right)_{3}$ is a weak equivalence. Hence the simplicial group $U\left(D_{k} B\right)^{\times}$acts by conjugation on the space

$$
\operatorname{Ring}\left(H \mathbb{Z}, D_{k} B\right)=\operatorname{map}_{\mathcal{G} R}\left(H \mathbb{Z}^{\mathrm{c}},\left(D_{k} B\right)_{3}\right)
$$

extending the action of the group $\Phi_{k}(B)$. As in (5.1) we denote by

$$
\operatorname{Ring}\left(H \mathbb{Z}, D_{k} B\right) / \text { conj. }
$$

the homotopy orbit space of $\operatorname{Ring}\left(H \mathbb{Z}, D_{k} B\right)$ by the conjugation action of $U\left(D_{k} B\right)_{1}^{\times}$, the identity component of $U\left(D_{k} B\right)^{\times}$. 
Since the Constructions 4.2 and 5.1 can be made functorial, truncations induce compatible maps

$$
\begin{aligned}
\operatorname{Ring}(H \mathbb{Z}, D B) & \longrightarrow \operatorname{Ring}\left(H \mathbb{Z}, D_{k} B\right) \\
\text { and } \quad \operatorname{Ring}\left(H \mathbb{Z}, D_{k} B\right) & \longrightarrow \operatorname{Ring}\left(H \mathbb{Z}, D_{k-1} B\right)
\end{aligned}
$$

and similarly for the orbit spaces by the conjugation actions.

Lemma 6.1 The maps

$$
\operatorname{Ring}(H \mathbb{Z}, D B) \longrightarrow \operatorname{holim}_{k} \operatorname{Ring}\left(H \mathbb{Z}, D_{k} B\right)
$$

and

$$
\operatorname{Ring}(H \mathbb{Z}, D B) / \operatorname{conj} . \longrightarrow \operatorname{holim}_{k}\left(\operatorname{Ring}\left(H \mathbb{Z}, D_{k} B\right) / \text { conj. }\right)
$$

induced by truncation are weak equivalences.

Proof We apply the homotopy limit construction [9] objectwise to Gammarings to obtain a construction of homotopy limits for Gamma-rings. As we explained in the proof of Theorem 3.2 (a), the homotopy fibre of the projection $D B \longrightarrow D_{k} B$ is the product of certain $\Gamma$-spaces $\left(B \otimes \mathcal{S}^{m}\right)^{!}$for $m>k$, each of which is $(2 k-1)$-connected. Hence the map

$$
(D B)_{3} \longrightarrow \operatorname{holim}_{k}\left(D_{k} B\right)_{3}
$$

is a stable equivalence of Gamma-rings. So after taking homomorphism spaces from $H \mathbb{Z}^{\mathrm{c}}$ we obtain a weak equivalence of spaces

$$
\begin{aligned}
\operatorname{map}_{\mathcal{G R}}\left(H \mathbb{Z}^{\mathrm{c}},(D B)_{3}\right) \stackrel{\sim}{\longrightarrow} & \operatorname{map}_{\mathcal{G R}}\left(H \mathbb{Z}^{\mathrm{c}}, \operatorname{holim}_{k}\left(D_{k} B\right)_{3}\right) \\
& \cong \operatorname{holim}_{k} \operatorname{Ring}\left(H \mathbb{Z}, D_{k} B\right)
\end{aligned}
$$

which proves the first statement. Similarly, the induced map of homotopy units

$$
(D B)^{\times} \longrightarrow \operatorname{holim}_{k}\left(D_{k} B\right)^{\times}
$$

is a weak equivalence, and so also the homotopy orbit space $\operatorname{Ring}(H \mathbb{Z}, D B) /$ conj. is the homotopy inverse limit of the truncated versions.

On the formal group law side, the classifying space $\mathcal{F} \mathcal{G}^{\text {str }}(B)$ can also be expressed as a homotopy limit of suitable truncated versions. We denote by $\mathcal{B} u d_{B}^{k}$ the classifying space of the groupoid of $k$-buds [28, Def. 2.1] (also called $k$-jets) of formal group laws over $B$ and $k$-buds of strict isomorphisms. Truncation induces maps

$$
\mathcal{F} \mathcal{G} \mathcal{L}^{\text {str }}(B) \longrightarrow \mathcal{B} u d_{B}^{k} \quad \text { and } \quad \mathcal{B} u d_{B}^{k} \longrightarrow \mathcal{B} u d_{B}^{k-1} .
$$


The classifying spaces $\mathcal{F} \mathcal{G} \mathcal{L}^{\operatorname{str}}(B)$ and $\mathcal{B} u d_{B}^{k}$ are isomorphic to the homotopy orbit spaces $\operatorname{FGL}(B)_{h \Phi(B)}$ and $\left(\operatorname{Bud}_{B}^{k}\right)_{h \Phi_{k}(B)}$ respectively. Since the group $\Phi(B)$ is the inverse limit of the groups $\Phi_{k}(B)$ and the set of formal group laws is the inverse limit of the sets of $k$-buds, we have

Lemma 6.2 The map

$$
\mathcal{F} \mathcal{G L}^{\text {str }}(B) \longrightarrow \operatorname{holim}_{k} \mathcal{B} u d_{B}^{k}
$$

induced by truncation on classifying spaces is a weak equivalence.

Proof This an instance of a general fact about homotopy orbits of groups acting on sets, alias translation categories. Suppose $\left\{G_{k} \longrightarrow G_{k-1}\right\}_{k \geq 1}$ is a sequence of surjective group homomorphisms, $\left\{X_{k} \longrightarrow X_{k-1}\right\}_{k \geq 1}$ a tower of sets, and suppose that $G_{k}$ acts on $X_{k}$ in such a way that the map $X_{k} \longrightarrow X_{k-1}$ is $G_{k}$-equivariant. Then the inverse limit $G=\lim _{k} G_{k}$ of groups acts on the inverse limit $X=\lim _{k} X_{k}$ of sets and the canonical map

$$
X_{h G} \longrightarrow \operatorname{holim}_{k}\left(X_{k}\right)_{h G_{k}}
$$

is a weak equivalence. This follows from the homotopy fibre sequences

$$
X_{k} \longrightarrow\left(X_{k}\right)_{h G_{k}} \longrightarrow B G_{k}
$$

by passage to homotopy inverse limit together with the fact that the natural maps

$$
X=\lim _{k} X_{k} \longrightarrow \operatorname{holim}_{k} X_{k} \quad \text { and } \quad B G \longrightarrow \operatorname{holim}_{k} B G_{k}
$$

are weak equivalences (we note that the path components of the homotopy inverse limit of the classifying spaces $B G_{k}$ are in bijective correspondence with the set $\lim _{k}^{1} G_{k}$; since we consider surjective group homomorphisms, this $\lim ^{1}$ term is trivial and the map from $B G$ to the homotopy inverse limit is indeed a weak equivalence). In our example $X_{k}$ is the set of $k$-buds of formal group laws and $G_{k}$ is the group $\Phi_{k}(B)$ of $k$-buds of power series conjugating the $k$-buds of formal group laws.

Every $k$-bud of formal group law $F$ gives rise to a map of Gamma-rings $H \mathbb{Z} \longrightarrow$ $D_{k} B$ in the same fashion as genuine formal group laws give maps to $D B$ (3.5). The group $\Phi_{k}(B)$ of truncated invertible power series conjugates the set of $k$-buds and the map $\operatorname{Bud}_{B}^{k} \longrightarrow \operatorname{map}_{\mathcal{G R}}\left(H \mathbb{Z}, D_{k} B\right)$ is $\Phi_{k}(B)$-equivariant. If we carry out Construction 5.1 with $R=D_{k} B$ and with the group $\Phi_{k}(B)$ 
conjugating the $k$-buds of formal group laws, we obtain a map of homotopy orbit spaces

$$
\begin{aligned}
\kappa_{k}:{\widetilde{\mathcal{B} u d_{B}^{k}}}^{k}={\left.\widetilde{\left(\operatorname{Bud}_{B}^{k}\right.}\right)_{h \Phi_{k}(B)}}^{\longrightarrow} \operatorname{map}_{\mathcal{G R}}\left(H \mathbb{Z}^{\mathrm{c}},\left(D_{k} B\right)_{3}\right)_{h U\left(D_{k} B\right)_{1}^{\times}} \\
=\operatorname{Ring}\left(H \mathbb{Z}, D_{k} B\right) / \text { conj. }
\end{aligned}
$$

The constructions are natural, so we end up with a commutative diagram

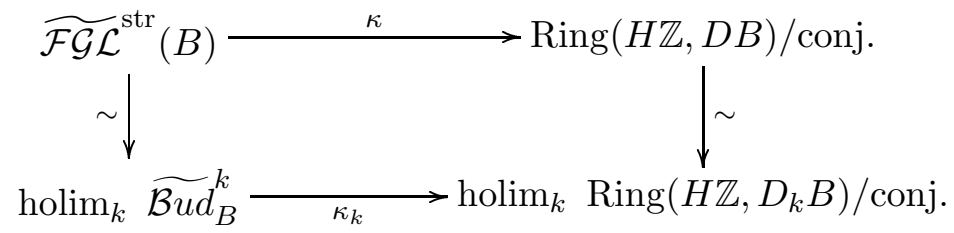

in which the vertical maps are weak equivalences by Lemmas 6.1 and 6.2, So Theorem 5.2 follows once we have shown

Theorem 6.4 For all $k \geq 1$ the map

$$
\kappa_{k}: \widetilde{\mathcal{B} u d}_{B}^{k} \longrightarrow \operatorname{Ring}\left(H \mathbb{Z}, D_{k} B\right) / \text { conj. }
$$

is a weak equivalence.

Remark 6.5 If $k$ is not a prime power, then the functor $B \otimes \mathcal{S}^{k}$ is a retract of a diagonalizable functor (Lemma 12.5), and so the $\Gamma$-space underlying $\left(B \otimes \mathcal{S}^{k}\right)^{\text {! }}$ is stably contractible 8.9 (e)). Hence the reduction map $D_{k} B \longrightarrow D_{k-1} B$ is a stable equivalence of Gamma-rings and the induced map on homotopy units $\left(D_{k} B\right)^{\times} \longrightarrow\left(D_{k-1} B\right)^{\times}$is a weak equivalence of simplicial monoids. Thus the map of homotopy orbit spaces

$$
\operatorname{Ring}\left(H \mathbb{Z}, D_{k} B\right) / \text { conj. } \stackrel{\text { red. }}{\longrightarrow} \operatorname{Ring}\left(H \mathbb{Z}, D_{k-1} B\right) / \text { conj. }
$$

is a weak equivalence. By Remark 7.3 below, the reduction functor $\mathcal{B} u d_{B}^{k} \longrightarrow$ $\mathcal{B} u d_{B}^{k-1}$ is an equivalence of categories. Hence in the inductive step nothing happens unless $k=p^{h}$ is a prime power. However it is convenient to make this case distinction only at the very end (see Step 1 in the proof of Theorem 12.3).

\section{Some singular extensions of Gamma-rings}

In this section we start the inductive proof of Theorem 6.4 We exploit that the truncation maps $D_{k} B \longrightarrow D_{k-1} B$ are "singular extensions" of Gamma-rings. This lets us reduce the problem to a comparison of the derivation space of the 
Gamma-ring $H \mathbb{Z}$ with coefficients in the "kernel" $\left(B \otimes \mathcal{S}^{k}\right)$ ! of the extension to the groupoid of symmetric 2-cocycles.

The case $k=1$ of Theorem 6.4 is straightforward. There is only one 1-bud of formal group law, and the only 1-bud of strict automorphism is the identity. On the other hand, $D_{1} B$ is isomorphic to the Eilenberg-MacLane Gamma-ring $H B$, so both the space $\operatorname{Ring}\left(H \mathbb{Z}, D_{1} B\right)$ and the unit component $\left(D_{1} B\right)_{1}^{\times}$of the homotopy units are weakly contractible. Hence source and target of the map

$$
\kappa_{1}: \widetilde{\mathcal{B} u}_{B}^{1} \longrightarrow \operatorname{Ring}\left(H \mathbb{Z}, D_{1} B\right) / \text { conj. }
$$

are weakly contractible and $\kappa_{1}$ is a weak equivalence.

7.1 Symmetric 2-cocycles For the inductive step we recall how the difference between $k$-buds and $(k-1)$-buds of formal group laws is controlled by symmetric 2 -cocycles. A symmetric 2 -cocycle of degree $k$ with values in $B$ is a homogeneous polynomial $c(x, y) \in B[x, y]$ of degree $k$ which satisfies the relations

$$
c(x, y)=c(y, x) \text { and } c(x, y)+c(x+y, z)=c(x, y+z)+c(y, z) .
$$

If $F$ is any $k$-bud of formal group law over $B$ and $c$ is a $k$-homogenous 2cocycle, then the truncated power series $F(x, y)+c(x, y)$ is another $k$-bud of formal group law with the same $(k-1)$-bud as $F$. Conversely, if $F$ and $F^{\prime}$ are two $k$-buds with the same reduction modulo the $k$-th powers of the augmentation ideal, then $c=F-F^{\prime}$ is a $k$-homogenous $2-$ cocycle. The proof of this is straightforward, compare [28, Sec. II] or [19, III.1 Lemma 2].

We define $\mathcal{Z}\left(B \otimes \mathcal{S}^{k}\right)$, the groupoid of symmetric 2-cocycles of degree $k$, as the category whose objects are the symmetric 2 -cocycles of degree $k$ over $B$. The set of morphisms from a cocycle $c$ to a cocycle $c^{\prime}$ consists of those $b \in B$ satisfying $c^{\prime}=c+b \cdot\left[x^{k}+y^{k}-(x+y)^{k}\right]$; composition is given by addition in $B$.

Suppose $F$ is a $k$-bud of formal group law. Then we can define a functor

$$
F+-: \mathcal{Z}\left(B \otimes \mathcal{S}^{k}\right) \longrightarrow \mathcal{B} u d_{B}^{k}
$$

on objects by $(F+-)(c)=F+c$ and on morphisms by $(F+-)(b)=x+b \cdot x^{k}$. The relation

$$
F^{x+b x^{k}}(y, \bar{y}) \equiv F(y, \bar{y})+b \cdot\left[y^{k}+\bar{y}^{k}-(y+\bar{y})^{k}\right] \quad \bmod (y, \bar{y})^{k+1}
$$

shows that if $b: c \longrightarrow c^{\prime}$ is a morphism of cocycles, then the power series $x+b \cdot x^{k}$ is indeed an isomorphism from $F+c$ to $F+c^{\prime}$. 
Lemma 7.2 For every $k$-bud of formal group law $F$, the functor

$$
F+-: \mathcal{Z}\left(B \otimes \mathcal{S}^{k}\right) \longrightarrow \mathcal{B} u d_{B}^{k}
$$

induces a weak equivalence from the classifying space of the groupoid $\mathcal{Z}\left(B \otimes \mathcal{S}^{k}\right)$ to the homotopy fibre of the truncation map $\mathcal{B} u d_{B}^{k} \longrightarrow \mathcal{B} u d_{B}^{k-1}$ over the basepoint $F$.

Proof This again is an instance of a general fact about homotopy orbits of groups acting on sets, alias translation categories. Suppose $G \longrightarrow \bar{G}$ is an epimorphism of groups with kernel $K$. Moreover, let $X$ be a $G$-set, $\bar{X}$ a $\bar{G}$-set and $\pi: X \longrightarrow \bar{X}$ a $G$-equivariant map. Then for every point $x \in \bar{X}$ the kernel $K$ acts on the preimage $\pi^{-1}(x)$. In this situation the sequence of homotopy orbit spaces

$$
\pi^{-1}(x)_{h K} \longrightarrow X_{h G} \longrightarrow \bar{X}_{h \bar{G}}
$$

is a homotopy fibre sequence over the point $x$.

In the situation at hand the epimorphism is the truncation $\Phi_{k}(B) \longrightarrow \Phi_{k-1}(B)$, whose kernel is isomorphic to the additive group of $B$ via $b \longmapsto x+b \cdot x^{k}$. The groups conjugate the $k$-buds respectively $(k-1)$-buds of formal group laws. For every choice of $k$-bud of formal group law $F$, the map $F+-$ is an isomorphism, equivariant for $B \cong$ kernel: $\Phi_{k}(B) \longrightarrow \Phi_{k-1}(B)$, from the symmetric 2 -cocycles to the $k$-buds which have the same $(k-1)$-bud as $F$. Hence the lemma follows.

Remark 7.3 The symmetric 2-cocycles have been identified by Lazard 28, II Lemme 3], see also [19, III.1 Thm. 1a]. There is a universal integral symmetric 2 -cocycle $c_{k} \in \mathbb{Z}[x, y]$ of degree $k$ given by

$$
c_{k}(x, y)=\frac{1}{d_{k}}\left[x^{k}+y^{k}-(x+y)^{k}\right]
$$

and the degree $k$ symmetric $2-$ cocycles over $B$ are precisely the multiples $b \cdot c_{k}$ for $b \in B$. Here $d_{k}$ is the greatest common divisor of the binomial coefficients $\left(\begin{array}{l}k \\ i\end{array}\right)$ for $1 \leq i \leq k-1$, which evaluates to

$$
d_{k}= \begin{cases}p & \text { if } k=p^{h} \text { for a prime } p \text { and } h \geq 1 \\ 1 & \text { else. }\end{cases}
$$

Hence the classifying space of $\mathcal{Z}\left(B \otimes \mathcal{S}^{k}\right)$ can be identified as follows: if $k$ is not a prime power, then the classifying space is weakly contractible and the reduction functor $\mathcal{B} u d_{B}^{k} \longrightarrow \mathcal{B} u d_{B}^{k-1}$ is an equivalence of categories. If $k=p^{h}$ 
for some prime $p$, then the groupoid $\mathcal{Z}\left(B \otimes \mathcal{S}^{p^{h}}\right)$ is isomorphic to the translation category of the action of $B$ on itself given by

$$
(b, x) \longmapsto p \cdot b+x .
$$

Hence the components of the classifying space of $\mathcal{Z}\left(B \otimes \mathcal{S}^{p^{h}}\right)$ are in bijective correspondence with the set $B / p B$ and the fundamental group at each basepoint is isomorphic to the group of those $b \in B$ such that $p b=0$. However, in the rest of this paper we will not use this explicit knowledge about the symmetric $2-$ cocycles.

Now we identify the difference between the spaces of Gamma-ring maps from $H \mathbb{Z}$ to $D_{k} B$ and to $D_{k-1} B$, ie, we study the homotopy fibers of the reduction map

$$
\operatorname{Ring}\left(H \mathbb{Z}, D_{k} B\right) / \text { conj. } \longrightarrow \operatorname{Ring}\left(H \mathbb{Z}, D_{k-1} B\right) / \text { conj. }
$$

For this purpose we consider the Gamma-ring $H \mathbb{Z} \times\left(B \otimes \mathcal{S}^{k}\right)^{!}$. We present the construction in a more general context, since we need it later.

7.4 Split singular extensions Let $G$ be a functor from the category of finitely generated free abelian groups to the category of all abelian groups, and suppose that $G$ is reduced in the sense that $G(0) \cong 0$. We define a Gamma-ring $H \mathbb{Z} \times G^{!}$, the split singular extension of $H \mathbb{Z}$ by the bimodule $G^{!}$.

First there is an $H \mathbb{Z}$-bimodule $G^{!}$associated to the functor $G$; the notation is taken from [37, Ex. 2.6], where the construction first appeared. As a $\Gamma$-space, $G^{!}$is the composite

$$
\Gamma^{\mathrm{op}} \stackrel{\widetilde{\mathbb{Z}}}{\longrightarrow} \text { (f. g. free ab. groups) } \stackrel{G}{\longrightarrow} \mathcal{A} b \stackrel{\Phi}{\longrightarrow} \text { (pt. sets) }
$$

of the reduced free functor $\widetilde{\mathbb{Z}}$, followed by $G$ and the forgetful functor $\Phi$ from abelian groups to pointed sets. The $\Gamma$-space $G^{!}$has the structure of an $H \mathbb{Z}^{-}$ bimodule via the composite

$$
H \mathbb{Z} \wedge G^{!} \wedge H \mathbb{Z} \stackrel{\text { assembly }}{\longrightarrow} H \mathbb{Z} \circ G^{!} \circ H \mathbb{Z} \longrightarrow G^{!} .
$$

The first map is an instance of the assembly map (2.5) from the smash product to the composition product of $\Gamma$-spaces; the second map is induced by evaluation maps (this uses that the original functor $G$ was defined for and takes values in abelian groups).

The product $H \mathbb{Z} \times G^{!}$becomes a Gamma-ring as follows. The unit map of $H \mathbb{Z} \times G^{!}$is the composition of the unit $\eta: \mathbb{S} \longrightarrow H \mathbb{Z}$ with the inclusion $H \mathbb{Z} \longrightarrow$ 
$H \mathbb{Z} \times G^{!}$. The multiplication map is the composite

$$
\begin{aligned}
& \left(H \mathbb{Z} \times G^{!}\right) \wedge\left(H \mathbb{Z} \times G^{!}\right) \longrightarrow(H \mathbb{Z} \wedge H \mathbb{Z}) \times\left(H \mathbb{Z} \wedge G^{!}\right) \times\left(G^{!} \wedge H \mathbb{Z}\right) \\
& \stackrel{\left(\mu_{H \mathbb{Z}}, l+r\right)}{\longrightarrow} \quad H \mathbb{Z} \times G^{!}
\end{aligned}
$$

where $l$ and $r$ denote the left respectively right action of $H \mathbb{Z}$ on $G^{!}$.

In particular we can apply Construction 7.4 to the functor $B \otimes \mathcal{S}^{k}$ which takes a finitely generated free abelian group $A$ to the tensor product of $B$ with the $k$-th symmetric power of $A$. If $F$ is any $k$-bud of formal group law, then $F_{*}: H \mathbb{Z} \longrightarrow D_{k} B$ is a morphism of Gamma-rings. Homogenous polynomials of degree $k$ naturally inject into the quotient of power series by terms of degree $k+1$, which gives a map of $\Gamma$-spaces Incl: $\left(B \otimes \mathcal{S}^{k}\right)^{!} \longrightarrow D_{k} B$. Moreover, their pointwise sum in $D_{k} B$

$$
F_{*}+\text { Incl. : } H \mathbb{Z} \times\left(B \otimes \mathcal{S}^{k}\right)^{!} \longrightarrow D_{k} B
$$

is again a map of Gamma-rings.

Lemma 7.5 The commutative square

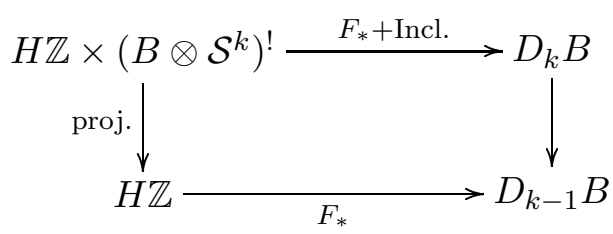

is a homotopy fibre square of Gamma-rings.

Proof It suffices to show that the underlying square of $\Gamma$-spaces is homotopy cartesian. As a $\Gamma$-space $D B$ splits as a product

$$
D_{k} B \cong D_{k-1} B \times\left(B \otimes \mathcal{S}^{k}\right) !
$$

and under this isomorphism the map $F_{*}+$ Incl. becomes the map

$$
F_{*} \times \mathrm{Id}: H \mathbb{Z} \times\left(B \otimes \mathcal{S}^{k}\right)^{!} \longrightarrow D_{k-1} B \times\left(B \otimes \mathcal{S}^{k}\right)^{!},
$$

so the claim follows.

The additive group of the ring $B$ includes into the underlying monoid of the Gamma-ring $H \mathbb{Z} \times\left(B \otimes \mathcal{S}^{k}\right)^{!}$via the map which sends $b \in B$ to the polynomial $x+b \cdot x^{k}$, considered as an element of $\left(H \mathbb{Z} \times\left(B \otimes \mathcal{S}^{k}\right)^{!}\right)\left(1^{+}\right) \cong \mathbb{Z}[x] \times B\left[x^{k}\right]$ (where $x$ is an indeterminate corresponding to the non-basepoint element of $\left.1^{+}\right)$. We can now apply Construction 4.2 to the Gamma-ring $H \mathbb{Z} \times\left(B \otimes \mathcal{S}^{k}\right)^{\text {! }}$ 
relative to the additive group of $B$. The construction produces a commutative diagram of Gamma-rings

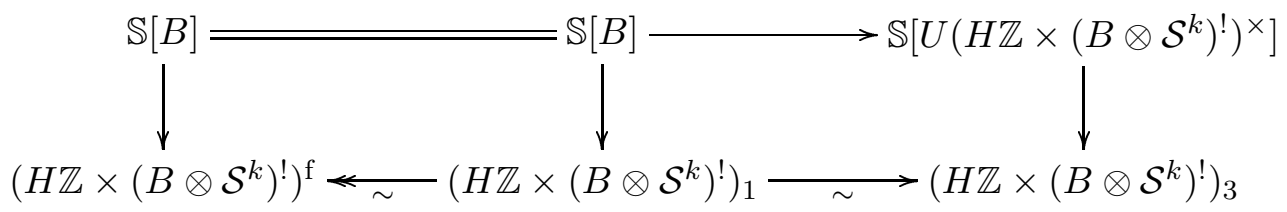

in which the lower horizontal maps are stable equivalences between fibrant Gamma-rings. The induced map from the simplicial group $U\left(H \mathbb{Z} \times\left(B \otimes \mathcal{S}^{k}\right)^{!}\right)^{\times}$ to the invertible components of the underlying monoid of $\left(H \mathbb{Z} \times\left(B \otimes \mathcal{S}^{k}\right)^{!}\right)_{3}$ is a weak equivalence.

We denote by

$$
\operatorname{der}\left(H \mathbb{Z}, B \otimes \mathcal{S}^{k}\right)=\operatorname{map}_{\mathcal{G} R}\left(H \mathbb{Z}^{\mathrm{c}},\left(H \mathbb{Z} \times\left(B \otimes \mathcal{S}^{k}\right)^{!}\right)_{3}\right)
$$

the space of Gamma-ring maps from $H \mathbb{Z}^{\mathrm{c}}$ to $\left(H \mathbb{Z} \times\left(B \otimes \mathcal{S}^{k}\right)^{!}\right)_{3}$ and refer to this space as the space of derivations of $H \mathbb{Z}$ with coefficients in $B \otimes \mathcal{S}^{k}$. The group $U\left(H \mathbb{Z} \times\left(B \otimes \mathcal{S}^{k}\right)^{!}\right)^{\times}$acts by conjugation on the space of derivations and we denote by $\operatorname{der}\left(H \mathbb{Z}, B \otimes \mathcal{S}^{k}\right) /$ conj. the homotopy orbit space of the conjugation action of the identity component $U\left(H \mathbb{Z} \times\left(B \otimes \mathcal{S}^{k}\right)^{!}\right)_{1}^{\times}$. Since the square of monoids

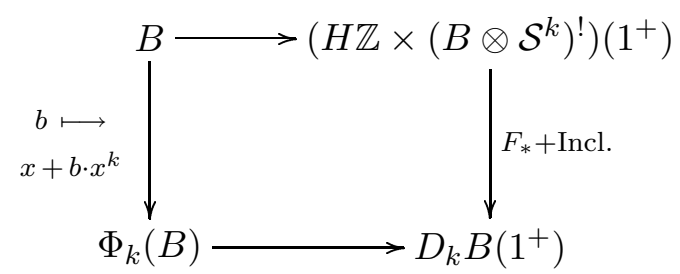

commutes and the constructions of Sections 4 are functorial in Gamma-rings equipped with a map from a group to the underlying monoid, the map

$$
F_{*}+\text { Incl. : } H \mathbb{Z} \times\left(B \otimes \mathcal{S}^{k}\right)^{!} \longrightarrow D_{k} B
$$

induces maps between the respective derived spaces of Gamma-ring maps from $H \mathbb{Z}$ and their homotopy orbit spaces.

Lemma 7.6 The two maps

$$
\begin{array}{cll}
\operatorname{der}\left(H \mathbb{Z}, B \otimes \mathcal{S}^{k}\right) & \stackrel{F_{*}+\text { Incl. }}{\longrightarrow} \operatorname{Ring}\left(H \mathbb{Z}, D_{k} B\right) \quad \text { and } \\
\operatorname{der}\left(H \mathbb{Z}, B \otimes \mathcal{S}^{k}\right) / \text { conj. } & \stackrel{F_{*}+\text { Incl. }}{\longrightarrow} & \operatorname{Ring}\left(H \mathbb{Z}, D_{k} B\right) / \text { conj. }
\end{array}
$$


induce weak equivalences between the derivation space, respectively its homotopy orbit space, and the respective homotopy fibres of the truncation maps

$$
\begin{array}{cl}
\operatorname{Ring}\left(H \mathbb{Z}, D_{k} B\right) & \longrightarrow \operatorname{Ring}\left(H \mathbb{Z}, D_{k-1} B\right) \quad \text { and } \\
\operatorname{Ring}\left(H \mathbb{Z}, D_{k} B\right) / \text { conj. } & \longrightarrow \operatorname{Ring}\left(H \mathbb{Z}, D_{k-1} B\right) / \text { conj. }
\end{array}
$$

over the basepoint $F_{*}$.

Proof The first statement is a direct consequence of the fact that the square of Lemma 7.5 is homotopy cartesian. The second follows from the first since the sequence of simplicial groups

$$
U\left(H \mathbb{Z} \times\left(B \otimes \mathcal{S}^{k}\right)^{!}\right)_{1}^{\times} \longrightarrow U\left(D_{k} B\right)_{1}^{\times} \longrightarrow U\left(D_{k-1} B\right)_{1}^{\times}
$$

is also a homotopy fibre sequence, again because of Lemma 7.5

We denote by $\widetilde{\mathrm{Z}}_{s}^{2}\left(B \otimes \mathcal{S}^{k}\right)$ the pullback of the diagram

$$
\operatorname{Z}_{s}^{2}\left(B \otimes \mathcal{S}^{k}\right) \longrightarrow \operatorname{map}_{\mathcal{G R}}\left(H \mathbb{Z}^{\mathrm{c}},\left(H \mathbb{Z} \times\left(B \otimes \mathcal{S}^{k}\right)^{!}\right)_{1}\right)
$$

All maps are equivariant with respect to the action of the additive group of $B$, so this group acts on $\widetilde{\mathrm{Z}}_{s}^{2}\left(B \otimes \mathcal{S}^{k}\right)$. Furthermore the weak equivalence

$$
\operatorname{map}_{\mathcal{G R}}\left(H \mathbb{Z}^{\mathrm{c}},\left(H \mathbb{Z} \times\left(B \otimes \mathcal{S}^{k}\right)\right)_{1}\right) \stackrel{\sim}{\longrightarrow} \operatorname{map}_{\mathcal{G R}}\left(H \mathbb{Z}^{\mathrm{c}},\left(H \mathbb{Z} \times\left(B \otimes \mathcal{S}^{k}\right)\right)_{3}\right)
$$

is $B$-equivariant, and on the target the action extends to an action by the simplicial group $U\left(H \mathbb{Z} \times\left(B \otimes \mathcal{S}^{k}\right)\right)_{1}^{\times}$. So we get an induced map on homotopy orbits

$$
\begin{aligned}
\kappa_{B \otimes \mathcal{S}^{k}}: \widetilde{\mathbb{Z}}_{s}^{2}\left(B \otimes \mathcal{S}^{k}\right)_{h B} & \longrightarrow \operatorname{map}_{\mathcal{G R}}\left(H \mathbb{Z}^{\mathrm{c}},\left(H \mathbb{Z} \times\left(B \otimes \mathcal{S}^{k}\right)^{!}\right)_{1}\right)_{h B} \longrightarrow \\
& \operatorname{map}_{\mathcal{G R}}\left(H \mathbb{Z}^{\mathrm{c}},\left(H \mathbb{Z} \times\left(B \otimes \mathcal{S}^{k}\right)^{!}\right)_{3}\right)_{h U\left(H \mathbb{Z} \times\left(B \otimes \mathcal{S}^{k}\right)^{!}\right)_{1}^{\times}} \\
& =\operatorname{der}\left(H \mathbb{Z}, B \otimes \mathcal{S}^{k}\right) / \text { conj. }
\end{aligned}
$$

Note that the homotopy orbit space of the action of $B$ on the set $\mathrm{Z}_{s}^{2}\left(B \otimes \mathcal{S}^{k}\right)$ of symmetric 2-cocycles is isomorphic to the classifying space of the groupoid $\mathcal{Z}\left(B \otimes \mathcal{S}^{k}\right)$ (7.1); hence we use the notation $\widetilde{\mathcal{Z}}\left(B \otimes \mathcal{S}^{k}\right)$ for the weakly equivalent homotopy orbit space $\widetilde{\mathrm{Z}}_{s}^{2}\left(B \otimes \mathcal{S}^{k}\right)_{h B}$.

Now we can reduce the inductive step of Theorem 6.4 to a statement about the map $\kappa_{B \otimes \mathcal{S}^{k}}$. We assume inductively that the map

$$
\kappa_{k-1}: \widetilde{\mathcal{B u d}}_{B}^{k-1} \longrightarrow \operatorname{Ring}\left(H \mathbb{Z}, D_{k-1} B\right) / \text { conj. }
$$


is a weak equivalence. This guarantees in particular that the $(k-1)$-buds of formal group laws account for all components of the target space. Since every $(k-1)$-bud of formal group law extends to a $k$-bud, the reduction map

$$
\operatorname{Ring}\left(H \mathbb{Z}, D_{k} B\right) / \text { conj. } \longrightarrow \operatorname{Ring}\left(H \mathbb{Z}, D_{k-1} B\right) / \text { conj. }
$$

is surjective on components. If we fix a $k$-bud of $F$ of formal group law, then the diagram

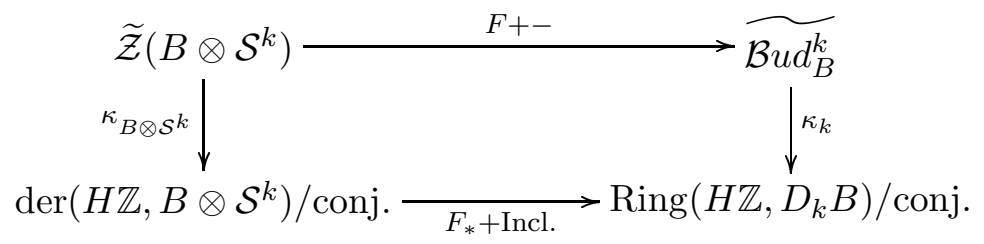

is commutative. By Lemmas 7.2 and [7.6 the horizontal maps identify the respective homotopy fibres of the truncation maps over the basepoints $F$ and $F_{*}$. Since formal group laws account for all components of the target space, we have thus reduced the inductive step of the proof of Theorem 6.4, and hence of the main theorem, to showing

Theorem 7.8 The map

$$
\kappa_{B \otimes \mathcal{S}^{k}}: \tilde{\mathcal{Z}}\left(B \otimes \mathcal{S}^{k}\right) \longrightarrow \operatorname{der}\left(H \mathbb{Z}, B \otimes \mathcal{S}^{k}\right) / \text { conj. }
$$

is a weak equivalence for all commutative rings $B$ and all $k \geq 1$.

The remaining sections are spent verifying that the map $\kappa_{B \otimes \mathcal{S}^{k}}$ is indeed a weak equivalence. If $k$ is not a prime power, then source and target of the map $\kappa_{B \otimes \mathcal{S}^{k}}$ are weakly contractible, compare Remark 6.5. However there is no need to make this case distinction until the very end (see Step 1 in the proof of Theorem 12.3).

\section{Symmetric 2-cocycles and derivations}

In this section we provide some general constructions which will be needed in the sequel. We consider functors $G$ from finitely generated free abelian groups to all abelian groups which are reduced in the sense that $G(0) \cong 0$. For such functors we discuss symmetric 2-cocycles and show how these lead to Gammaring maps into the split extension $H \mathbb{Z} \times G^{!}$(17.4). We also recall the Dold-Puppe stabilization $G_{s t}$ of the functor $G$ (8.9). The work of this section is summarized in a certain map 8.10)

$$
\lambda_{G}: \mathcal{Z}(G) \longrightarrow \operatorname{map}_{\mathcal{G R}}\left(H \mathbb{Z}^{\mathrm{c}}, H \mathbb{Z} \times G_{s t}^{!}\right)_{h G_{s t}(\mathbb{Z})}
$$


with source the groupoid $\mathcal{Z}(G)$ of symmetric 2 -cocycles of $G$. In the case where $G$ is the symmetric power functor $B \otimes \mathcal{S}^{k}$, we identify the map $\lambda_{B \otimes \mathcal{S}^{k}}$ (up to weak equivalence) with the map $\kappa_{B \otimes \mathcal{S}^{k}}$ of Theorem 7.8 in Section 9

8.1 Cocycles of a functor We let $\mathcal{F}$ denote the abelian category of reduced functors from finitely generated free abelian groups to all abelian groups. Suppose $G \in \mathcal{F}$ is a such functor. Then a symmetric 2 -cocycle with values in $G$ is an element $c \in G(\mathbb{Z} \oplus \mathbb{Z})$ which is

(a) fixed by the involution of $G(\mathbb{Z} \oplus \mathbb{Z})$ induced by the interchange of summands and

(b) in the kernel of the map

$$
\begin{gathered}
G\left(\begin{array}{lll}
1 & 0 & 0 \\
0 & 1 & 0
\end{array}\right)-G\left(\begin{array}{lll}
1 & 0 & 0 \\
0 & 1 & 1
\end{array}\right)+G\left(\begin{array}{lll}
1 & 1 & 0 \\
0 & 0 & 1
\end{array}\right)-G\left(\begin{array}{lll}
0 & 1 & 0 \\
0 & 0 & 1
\end{array}\right): \\
G(\mathbb{Z} \oplus \mathbb{Z}) \longrightarrow G(\mathbb{Z} \oplus \mathbb{Z} \oplus \mathbb{Z}) .
\end{gathered}
$$

We denote by $\mathrm{Z}_{s}^{2}(G)$ the group of symmetric 2-cocycles in $G$.

A group homomorphism

$$
\theta: G(\mathbb{Z}) \longrightarrow \mathrm{Z}_{s}^{2}(G) \subset G(\mathbb{Z} \oplus \mathbb{Z})
$$

is defined by

$$
\theta=G(1,0)-G(1,1)+G(0,1) .
$$

The cocycle $\theta(a)$ associated to $a \in G(\mathbb{Z})$ is sometimes referred to as the principal cocycle of $a$. We denote by $\mathcal{Z}(G)$ the translation category of the action of $G(\mathbb{Z})$ on the set $\mathrm{Z}_{s}^{2}(G)$ of symmetric 2-cocycles given by $(a, c) \longmapsto \theta(a)+c$. More precisely, $\mathcal{Z}(G)$ is the groupoid whose objects are the symmetric 2cocycles $\mathrm{Z}_{s}^{2}(G)$ and where the set of morphisms from a cocycle $c$ to a cocycle $c^{\prime}$ consists of those elements $a \in G(\mathbb{Z})$ such that $c^{\prime}=\theta(a)+c$. Composition in $\mathcal{Z}(G)$ is given by addition in the group $G(\mathbb{Z})$.

Example 8.2 If $G=B \otimes \mathcal{S}^{k}$ is the symmetric power functor, then $G(\mathbb{Z} \oplus \mathbb{Z})$ is the group of homogenous polynomials of degree $k$ in two variables over the ring $B$. For $c \in\left(B \otimes \mathcal{S}^{k}\right)(\mathbb{Z} \oplus \mathbb{Z})$, the cocycle condition (a) translates into $c(x, y)=c(y, x)$ and condition (b) translates into the equation

$$
c(x, y)+c(x+y, z)=c(x, y+z)+c(y, z) .
$$

Hence the symmetric 2 -cocycles with values in $B \otimes \mathcal{S}^{k}$ coincide with the homogenous 2-cocycles of degree $k$ as defined in 7.1. Moreover, the group $\left(B \otimes \mathcal{S}^{k}\right)(\mathbb{Z})$ is isomorphic to the additive group of $B$ and the map

$$
\theta: B \cong\left(B \otimes \mathcal{S}^{k}\right)(\mathbb{Z}) \longrightarrow \mathrm{Z}_{s}^{2}\left(B \otimes \mathcal{S}^{k}\right)
$$


sends $b \in B$ to the cocycle $b \cdot\left[x^{k}+y^{k}-(x+y)^{k}\right]$. Hence the cocycle groupoid for the functor $B \otimes \mathcal{S}^{k}$ as defined in 8.1 coincides with $\mathcal{Z}\left(B \otimes \mathcal{S}^{k}\right)$ as defined in $[7.1]$

8.3 The universal 2-cocycle We let $I \in \mathcal{F}$ denote the inclusion functor and $P \in \mathcal{F}$ the functor which takes an abelian group $A$ to the reduced free abelian group generated by the underlying pointed set of $A$. The Yoneda isomorphism $\operatorname{Hom}_{\mathcal{F}}(P, G) \cong G(\mathbb{Z})$ shows that $P$ is a projective object of $\mathcal{F}$. Evaluation gives a natural epimorphism

$$
P(A)=\widetilde{\mathbb{Z}}[A] \longrightarrow A=I(A),
$$

ie, an epimorphism $\epsilon: P \longrightarrow I$ in the category $\mathcal{F}$. We let $J$ denote the kernel; the relevance for us is that $J$ represents the symmetric 2 -cocycle functor. The element

$$
c_{u}=[1,0]-[1,1]+[0,1] \in \widetilde{\mathbb{Z}}[\mathbb{Z} \oplus \mathbb{Z}]=P(\mathbb{Z} \oplus \mathbb{Z})
$$

is in the kernel of the evaluation map, so it is an element of $J(\mathbb{Z} \oplus \mathbb{Z})$. As an element of $P(\mathbb{Z} \oplus \mathbb{Z}), c_{u}$ is the principal cocycle associated to $[1] \in P(\mathbb{Z})$. Hence $c_{u}$ is a symmetric 2-cocycle of $J$ (but it is not principal for $J$ because the element $[1] \in P(\mathbb{Z})$ does not belong to $J(\mathbb{Z}))$.

Lemma 8.5 The symmetric 2-cocycle $c_{u}$ with values in the functor $J$ is universal in the sense that the map

$$
\operatorname{Hom}_{\mathcal{F}}(J, G) \longrightarrow \mathrm{Z}_{s}^{2}(G), \quad f \longmapsto f\left(c_{u}\right)
$$

is an isomorphism for all functors $G \in \mathcal{F}$.

Proof $\operatorname{Both} \operatorname{Hom}_{\mathcal{F}}(J, G)$ and $\mathrm{Z}_{s}^{2}(G)$ are additive and left exact in the functor $G$. Hence it suffices to check the claim for a set of injective cogenerators of the category $\mathcal{F}$. If $A$ is a finitely generated free abelian group we define a functor $\mathcal{I}_{A}$ by the formula

$$
\mathcal{I}_{A}(M)=\operatorname{map}_{*}(\operatorname{Hom}(M, A), \mathbb{Q} / \mathbb{Z}) .
$$

Here $\operatorname{Hom}(M, A)$ denotes the set of group homomorphisms from $M$ to $A$ and ' $\operatorname{map}_{*}$ ' refers to the group of set-theoretic maps from $\operatorname{Hom}(M, A)$ to $\mathbb{Q} / \mathbb{Z}$ preserving 0 , with group structure by pointwise addition. The Yoneda isomorphism

$$
\operatorname{Hom}_{\mathcal{F}}\left(G, \mathcal{I}_{A}\right) \cong \operatorname{Hom}(G(A), \mathbb{Q} / \mathbb{Z})
$$

implies that $\mathcal{I}_{A}$ is injective and that the functors $\mathcal{I}_{A}$ form a collection of injective cogenerators as $A$ varies. 
It remains to verify that for all finitely generated free abelian groups $A$ evaluation at the cocycle $c_{u} \in \mathrm{Z}_{s}^{2}(J)$ is an isomorphism from $\operatorname{Hom}_{\mathcal{F}}\left(J, \mathcal{I}_{A}\right)$ to $\mathrm{Z}_{s}^{2}\left(\mathcal{I}_{A}\right)$. We claim that the group $\mathrm{Z}_{s}^{2}\left(\mathcal{I}_{A}\right)$ can be identified with the quotient of the group $\operatorname{map}_{*}(A, \mathbb{Q} / \mathbb{Z})$ of pointed set maps from $A$ to $\mathbb{Q} / \mathbb{Z}$ by the subgroup $\operatorname{Hom}(A, \mathbb{Q} / \mathbb{Z})$ of additive maps. We use the natural basis of $\mathbb{Z} \oplus \mathbb{Z}$ to identify $\mathcal{I}_{A}(\mathbb{Z} \oplus \mathbb{Z})=\operatorname{map}_{*}(\operatorname{Hom}(\mathbb{Z} \oplus \mathbb{Z}, A), \mathbb{Q} / \mathbb{Z})$ with the group of set-theoretic maps $g: A \oplus A \longrightarrow \mathbb{Q} / \mathbb{Z}$ satisfying $g(0,0)=0$. Under this identification the cocycle conditions for elements of $\mathcal{I}_{A}(\mathbb{Z} \oplus \mathbb{Z})$ translate into the conditions

$$
g(x, y)=g(y, x) \text { and } g(x, y)+g(x+y, z)=g(x, y+z)+g(y, z)
$$

for all $x, y, z \in A$ on the function $g \in \operatorname{map}_{*}(A \oplus A, \mathbb{Q} / \mathbb{Z})$. In other words: $g$ is a factor set of an abelian group extension of $A$ by $\mathbb{Q} / \mathbb{Z}$. Since $A$ is free abelian, every such extension splits, so $g$ is principal, ie, there exists a function $h: A \longrightarrow \mathbb{Q} / \mathbb{Z}$ satisfying $g(x, y)=h(x)-h(x+y)+h(y)$ and $h(0)=0$. Moreover, $h$ is uniquely determined by this up to an additive function.

All this means that the bottom row in the commutative diagram of abelian groups

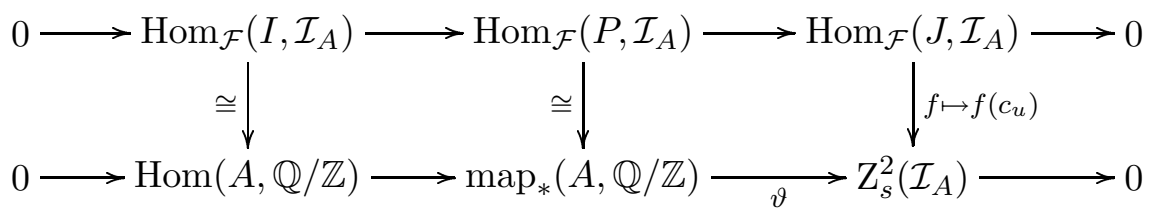

is exact where $\vartheta$ is defined by $\vartheta(h)(x, y)=h(x)-h(x+y)+h(y)$. The upper row is exact since $\mathcal{I}_{A}$ is an injective object and $J$ is the kernel of the epimorphism $\epsilon: P \longrightarrow I$. The left and middle vertical maps are special cases of the Yoneda isomorphism $\operatorname{Hom}_{\mathcal{F}}\left(G, \mathcal{I}_{A}\right) \cong \operatorname{Hom}(G(A), \mathbb{Q} / \mathbb{Z})$, so the right vertical map is also an isomorphism and $c_{u} \in \mathrm{Z}_{s}^{2}(J)$ is indeed a universal symmetric 2-cocycle.

8.6 The universal derivation We saw in Lemma 8.5 that the functor $J=\operatorname{kernel}(\epsilon: P \longrightarrow I)$ supports a universal symmetric 2-cocycle. Now we construct a universal derivation, ie, a certain homomorphism of Gamma-rings

$$
H \mathbb{Z} \longrightarrow H \mathbb{Z} \times J^{!}
$$

into the split extension (7.4) of $H \mathbb{Z}$ by $J^{!}$. The first component of this map is the identity map of $H \mathbb{Z}$. To describe the second component we consider the map of $\Gamma$-spaces

$$
\eta \circ 1-1 \circ \eta: H \mathbb{Z} \longrightarrow H \mathbb{Z} \circ H \mathbb{Z}
$$

where $\eta: \mathbb{S} \longrightarrow H \mathbb{Z}$ is the unit map of $H \mathbb{Z}$ given by inclusion of generators into the free abelian groups, and where we use the identifications $\mathbb{S} \circ H \mathbb{Z} \cong H \mathbb{Z} \cong$ 
$H \mathbb{Z} \circ \mathbb{S}$. We note that $H \mathbb{Z} \circ H \mathbb{Z}=P^{!}$and that the composite of $\eta \circ 1-1 \circ \eta$ with the evaluation map $\epsilon^{!}: H \mathbb{Z} \circ H \mathbb{Z}=P^{!} \longrightarrow I^{!}=H \mathbb{Z}$ is trivial. So we can define $d_{u}: H \mathbb{Z} \longrightarrow J^{!}$as the unique morphism of $\Gamma$-spaces whose composite with the inclusion $J^{!} \subseteq P^{!}$is the difference $\eta \circ 1-1 \circ \eta$. We refer to $d_{u}$ as the universal derivation. Now we claim that the map

$$
\left(1, d_{u}\right): H \mathbb{Z} \longrightarrow H \mathbb{Z} \times J^{!}
$$

is a homomorphism of Gamma-rings. The only thing to verify is the multiplicativity, and it suffices to do this after composition with the injective Gamma-ring map $H \mathbb{Z} \times J^{!} \longrightarrow H \mathbb{Z} \times P^{!}$induced by the inclusion $J \longrightarrow P$. By the definition of the product of $H \mathbb{Z} \times P^{!}=H \mathbb{Z} \times(H \mathbb{Z} \circ H \mathbb{Z})$ in 7.4 this boils down to verifying the commutativity of the diagram

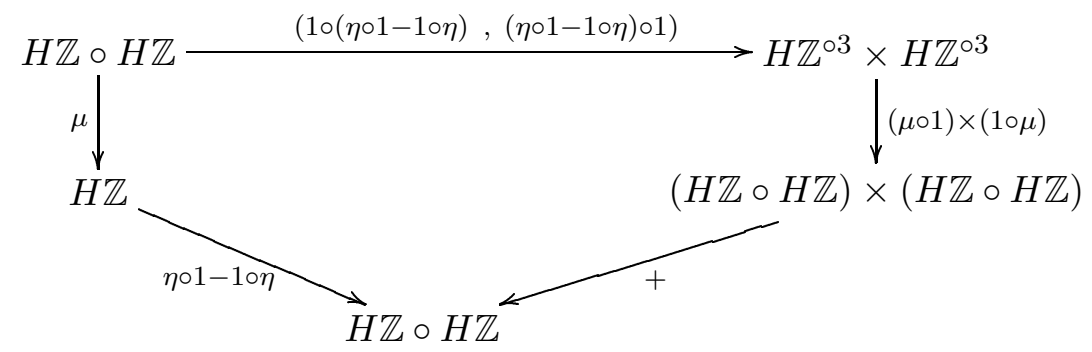

where $\mu: H \mathbb{Z} \circ H \mathbb{Z} \longrightarrow H \mathbb{Z}$ is the "multiplication" induced by the evaluation map $P \longrightarrow I$. The commutativity of the diagram in turn follows from the identities

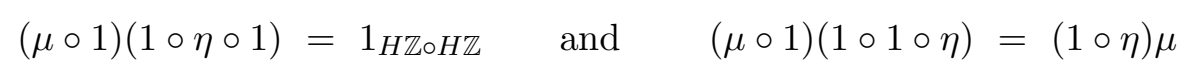

(here juxtaposition means composition of $\Gamma$-space maps) and their analogues for $\mu \circ 1$ replaced by $1 \circ \mu$.

Hence for a functor $G \in \mathcal{F}$ we can define a map

$$
\mathrm{Z}_{s}^{2}(G) \cong \operatorname{Hom}_{\mathcal{F}}(J, G) \longrightarrow \operatorname{map}_{\mathcal{G R}}\left(H \mathbb{Z}, H \mathbb{Z} \times G^{!}\right)
$$

by sending a morphism $f: J \longrightarrow G$ to the composite Gamma-ring map

$$
H \mathbb{Z} \stackrel{d_{u}}{\longrightarrow} H \mathbb{Z} \times J^{!} \stackrel{1 \times f^{!}}{\longrightarrow} H \mathbb{Z} \times G^{!} .
$$

If $c \in \mathrm{Z}_{s}^{2}(G)$ is a cocycle represented by $f_{c}: J \longrightarrow G$, then we refer to the above Gamma-ring map as the derivation associated to the 2-cocycle $c$.

The group $G(\mathbb{Z})$ maps to the underlying monoid $\left(H \mathbb{Z} \times G^{!}\right)\left(1^{+}\right) \cong \mathbb{Z} \times G(\mathbb{Z})$ of the split extension via $a \longmapsto(1, a)$, hence it acts on the Gamma-ring $H \mathbb{Z} \times G^{!}$ by conjugation (4.1). The group $G(\mathbb{Z})$ also acts on the symmetric 2-cocycles via the homomorphism $\theta: G(\mathbb{Z}) \longrightarrow \mathrm{Z}_{s}^{2}(G)$ (8.1). 
Lemma 8.8 The map

$$
\mathrm{Z}_{s}^{2}(G) \cong \operatorname{Hom}_{\mathcal{F}}(J, G) \longrightarrow \operatorname{map}_{\mathcal{G R}}\left(H \mathbb{Z}, H \mathbb{Z} \times G^{!}\right)
$$

which sends a morphism $f: J \longrightarrow G$ to the Gamma-ring map $\left(1, f^{!} \circ d_{u}\right)$ : $H \mathbb{Z} \longrightarrow H \mathbb{Z} \times G^{!}$is equivariant for the action of $G(\mathbb{Z})$.

Proof Let $a \in G(\mathbb{Z})$ be an element and $f: J \longrightarrow G$ a morphism of functors. Under the Yoneda isomorphism $G(\mathbb{Z}) \cong \operatorname{Hom}_{\mathcal{F}}(P, G)$ and the isomorphism $\mathrm{Z}_{s}^{2}(G) \cong \operatorname{Hom}_{\mathcal{F}}(J, G)$ of Lemma 8.5 the map $\theta: G(\mathbb{Z}) \longrightarrow \mathrm{Z}_{s}^{2}(G)$ corresponds to the map $\operatorname{Hom}_{\mathcal{F}}(P, G) \longrightarrow \operatorname{Hom}_{\mathcal{F}}(J, G)$ induced by the inclusion $\iota: J \longrightarrow P$.

So we have to verify the equality

$$
\left(1, d_{f+a \circ \iota}\right)=(1, a) \cdot\left(1, d_{f}\right) \cdot(1, a)^{-1}
$$

as maps $H \mathbb{Z} \longrightarrow H \mathbb{Z} \times G^{!}$. Only the second component matters. If we substitute definitions 4.1 of the conjugation action and 7.4 of the multiplication in $H \mathbb{Z} \times G^{!}$ we see that the second component of right hand side is a sum

$$
\operatorname{proj}_{G^{!}} \circ\left[(1, a) \cdot\left(1, d_{f}\right) \cdot(1, a)^{-1}\right]=r_{-a}+d_{f}+l_{a} .
$$

Here $l_{a}: H \mathbb{Z} \longrightarrow G^{!}$is left multiplication with $a \in G^{!}\left(1^{+}\right) \cong G(\mathbb{Z})$, ie, the composition

$$
H \mathbb{Z} \cong \mathbb{S} \wedge H \mathbb{Z} \stackrel{a \wedge 1}{\longrightarrow} G^{!} \wedge H \mathbb{Z} \stackrel{\text { action }}{\longrightarrow} G^{!},
$$

and $r_{-a}$ is right multiplication with $-a \in G^{!}\left(1^{+}\right)$.

Since $d_{f+a \circ \iota}=d_{f}+d_{a \circ \iota}$ it suffices to show that

$$
d_{a \circ \iota}=r_{-a}+l_{a}
$$

as maps $H \mathbb{Z} \longrightarrow G^{!}$. By naturality it is enough to check the universal example, ie, to take $G=P$ and $a=[1] \in \widetilde{\mathbb{Z}}[\mathbb{Z}]=P(\mathbb{Z})$, which corresponds to the identity of $P$ under the Yoneda isomorphism. By definition

$$
d_{\iota}=\eta \circ 1-1 \circ \eta: H \mathbb{Z} \longrightarrow P^{!}=H \mathbb{Z} \circ H \mathbb{Z} .
$$

So the claim follows since $l_{[1]}=\eta \circ 1$ and $r_{-[1]}=-1 \circ \eta$.

8.9 Dold-Puppe stabilization and MacLane's $Q$-construction We recall the Dold-Puppe stabilization of a functor $G \in \mathcal{F}$ (compare [11, 8.3]). We work with a specific model for the Dold-Puppe stabilization which uses MacLane's cubical construction [14, Sec. 12]. In the original paper of Eilenberg and MacLane the cubical construction was defined for the free functor $P=\widetilde{\mathbb{Z}}$, but the definition extends to arbitrary reduced functors in $\mathcal{F}$, see 35. Sec. 
4] or [27, 6.2] for the definition. A convenient reference for the relationship between Dold-Puppe stabilization and MacLane's cubical construction is [27]. The cubical construction $Q G$ of a functor $G \in \mathcal{F}$ is a chain complex of functors, concentrated in non-negative dimensions, with the following properties:

(a) $Q G$ is homotopy-additive in the sense that for every pair of finitely generated free abelian groups $A$ and $A^{\prime}$ the canonical map

$$
Q G(A) \oplus Q G\left(A^{\prime}\right) \longrightarrow Q G\left(A \oplus A^{\prime}\right)
$$

is a quasi-isomorphism.

(b) there is a natural isomorphism

$$
H_{*} Q G(\mathbb{Z}) \cong L_{*}^{\mathrm{st}} G \cong \pi_{*} G^{!},
$$

ie, the homology groups of the complex $Q G(\mathbb{Z})$ are isomorphic to the Dold-Puppe stable derived functors of $G$ and to the stable homotopy groups of the $\Gamma$-space $G^{!}$.

(c) In dimension zero, $(Q G)_{0}=G$ and in positive dimensions $Q G$ is a finite sum of higher order cross-effects (see [15, Sec.9] or [27, Sec. 7]) of $G$.

(d) As a functor of $G$, the assignment $G \longmapsto Q G$ is additive, exact, and commutes with limits and colimits.

(e) Suppose the functor $G$ is diagonalizable, ie, there exists a functor

$$
\bar{G}: \text { (f.g. free ab. groups) } \times(\text { f.g. free ab. groups }) \longrightarrow \mathcal{A} b
$$

of two variables satisfying $\bar{G}(A, 0) \cong 0 \cong \bar{G}(0, A)$ and a natural isomorphism $G(A) \cong \bar{G}(A, A)$. Then the complex $Q G$ is acyclic.

Property (a) is proved in [35, 4.2] and [27, 6.3]. The first isomorphism of part (b) follows from [35, 4.1] or [27, 7.5]; essentially by definition the stable homotopy groups of the $\Gamma$-space $G^{!}$are the Dold-Puppe stable derived functors of $G$. Part (c) is proved in [27, 6.3]. Property (d) follows from (c) since taking crosseffects is exact and commutes with limits and colimits. If $G$ is diagonalizable, then [11, 5.20] shows that the stable derived functors of $G$ are trivial. So by part (b) the complex $Q G(\mathbb{Z})$ is acyclic and by part (a) $Q G$ is acyclic as a complex of functors.

Properties (a) and (b) already characterize $Q G$ up to a chain of natural quasiisomorphisms; this is because on the level of homotopy categories, $G \longmapsto Q G$ is left adjoint to the inclusion of the subcategory of homotopy additive complexes of functors. 
We denote by $G_{s t}$ the simplicial functor which corresponds to $Q G$ under the Dold-Kan equivalence between simplicial objects and non-negative chain complexes in the abelian category $\mathcal{F}$. So $G_{s t}$ is defined by the property that its normalized chain complex is isomorphic to $Q G$. By property (c), the functor of zero-simplices of $G_{s t}$ is $G$, which gives a map $G \longrightarrow G_{s t}$ which induces isomorphism of stable homotopy groups upon passage to associated $\Gamma$-spaces $G^{!} \longrightarrow G_{s t}^{!}$.

Construction 7.4 which associates the Gamma-ring $H \mathbb{Z} \times G^{!}$to a functor $G \in \mathcal{F}$ makes perfect sense for simplicial functors, ie, simplicial objects in the abelian category $\mathcal{F}$. Moreover, the stabilization map $G \longrightarrow G_{s t}$ induces a stable equivalence of Gamma-rings $H \mathbb{Z} \times G^{!} \longrightarrow H \mathbb{Z} \times G_{s t}^{!}$. Combining the map (8.7) with this Gamma-ring homomorphism and the approximation map $H \mathbb{Z}^{\mathrm{c}} \longrightarrow H \mathbb{Z}$ gives a map

$$
\begin{aligned}
& \mathrm{Z}_{s}^{2}(G) \cong \operatorname{Hom}_{\mathcal{F}}(J, G) \stackrel{f \mapsto\left(1, f^{!} \circ d_{u}\right)}{\longrightarrow} \operatorname{map}_{\mathcal{G R}}\left(H \mathbb{Z}, H \mathbb{Z} \times G^{!}\right) \\
& \operatorname{map}_{\mathcal{G R}}\left(H \mathbb{Z}^{\mathrm{c}}, H \mathbb{Z} \times G_{s t}^{!}\right) .
\end{aligned}
$$

By Lemma 8.8 this map is equivariant for the action of $G(\mathbb{Z})$ by translation and conjugation respectively. Hence passing to homotopy orbits yields a map

$$
\lambda_{G}: \mathcal{Z}(G) \cong \mathrm{Z}_{s}^{2}(G)_{h G(\mathbb{Z})} \longrightarrow \operatorname{map}_{\mathcal{G R}}\left(H \mathbb{Z}^{\mathrm{c}}, H \mathbb{Z} \times G_{s t}^{!}\right)_{h G_{s t}(\mathbb{Z})} .
$$

Here we identified the classifying space of the groupoid $\mathcal{Z}(G)$ of symmetric 2cocycles (8.1) with the homotopy orbit space of the action of $G(\mathbb{Z})$ on the set $\mathrm{Z}_{s}^{2}(G)$.

\section{A change of models}

So far we have reduced the proof of our main theorem, Theorem [5.2, to the verification that the map

$$
\kappa_{B \otimes \mathcal{S}^{k}}: \widetilde{\mathcal{Z}}\left(B \otimes \mathcal{S}^{k}\right) \longrightarrow \operatorname{der}\left(H \mathbb{Z}, B \otimes \mathcal{S}^{k}\right) / \text { conj. }
$$

constructed in Section 7 is a weak equivalence for all $k \geq 1$. In this section we bring the map $\kappa_{B \otimes \mathcal{S}^{k}}$ into a more manageable form by constructing a commutative square

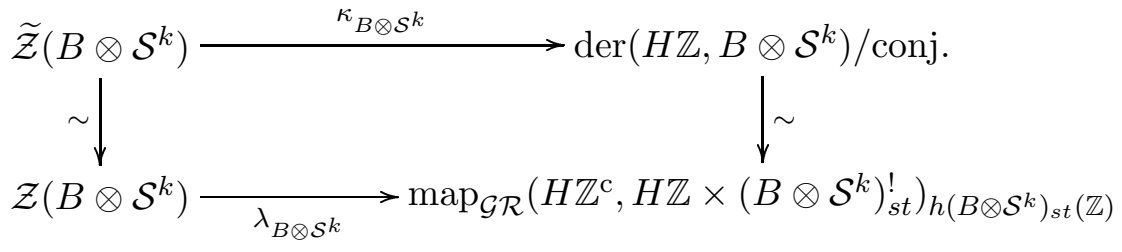


in which the vertical maps are weak equivalences. The lower horizontal map $\lambda_{B \otimes \mathcal{S}^{k}}$ is an instance of (8.10). This then leaves us with the task to verify that $\lambda_{B \otimes \mathcal{S}^{k}}$ is a weak equivalence for all $k \geq 1$.

The construction of the square (9.1) is technical. The idea is that by properties 8.9 (a) and (b) of the cubical construction, $\left(B \otimes \mathcal{S}^{k}\right)_{s t}^{!}$is a stably fibrant model of the $\Gamma$-space $\left(B \otimes \mathcal{S}^{k}\right)^{!}$. Hence the map $H \mathbb{Z} \times\left(B \otimes \mathcal{S}^{k}\right)^{!} \longrightarrow H \mathbb{Z} \times\left(B \otimes \mathcal{S}^{k}\right)_{s t}^{!}$ is a stable equivalence of Gamma-rings with fibrant target. In particular, the space of Gamma-ring maps $\operatorname{map}_{\mathcal{G} R}\left(H \mathbb{Z}^{\mathrm{c}}, H \mathbb{Z} \times\left(B \otimes \mathcal{S}^{k}\right)_{s t}^{!}\right)$is a model for the homotopy invariant morphism space, ie, it is weakly equivalent to the derivation space $\operatorname{der}\left(H \mathbb{Z}, B \otimes \mathcal{S}^{k}\right)$. In order to identify these two derivation spaces we have to take a little more care because we need to work relative to the classifying space of symmetric 2-cocycles.

We reexamine Construction 4.2, applied to the Gamma-ring $H \mathbb{Z} \times\left(B \otimes \mathcal{S}^{k}\right)^{\text {! }}$. This construction yields a commutative diagram of Gamma-rings

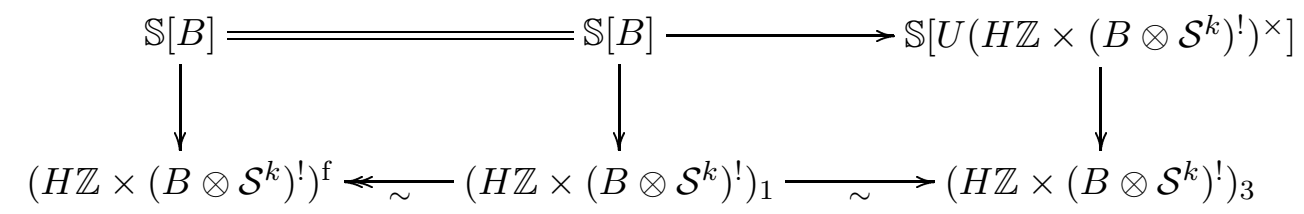

in which the lower horizontal maps are stable equivalences between stably fibrant Gamma-rings. Here we work relative to the homomorphism

$$
B \longrightarrow H \mathbb{Z} \times\left(B \otimes \mathcal{S}^{k}\right)^{!}\left(1^{+}\right)=\mathbb{Z}[x] \times B\left[x^{k}\right]
$$

which sends $b \in B$ to $x+b \cdot x^{k}$. Furthermore, the induced map from the simplicial group $U\left(H \mathbb{Z} \times\left(B \otimes \mathcal{S}^{k}\right)^{!}\right)^{\times}$to the invertible components of the underlying monoid of $\left(H \mathbb{Z} \times\left(B \otimes \mathcal{S}^{k}\right)^{!}\right)_{3}$ is a weak equivalence.

Since the Gamma-ring $H \mathbb{Z} \times\left(B \otimes \mathcal{S}^{k}\right)_{s t}^{!}$is stably fibrant and the map

$$
H \mathbb{Z} \times\left(B \otimes \mathcal{S}^{k}\right)^{!} \longrightarrow\left(H \mathbb{Z} \times\left(B \otimes \mathcal{S}^{k}\right)^{!}\right)^{\mathrm{f}}
$$

in the functorial fibrant replacement is an acyclic cofibration, we can choose a Gamma-ring map from $\left(H \mathbb{Z} \times\left(B \otimes \mathcal{S}^{k}\right)^{!}\right)^{\mathrm{f}}$ to $H \mathbb{Z} \times\left(B \otimes \mathcal{S}^{k}\right)_{s t}^{!}$under $H \mathbb{Z} \times$ $\left(B \otimes \mathcal{S}^{k}\right)^{\text {! }}$. This map will automatically be a stable equivalence. We can perform constructions 4.2 and 5.1 starting from either of these two fibrant replacements. The stable equivalence between them induces a weak equivalence between the two homotopy orbit spaces of the conjugation action. In other words, we can assume that the stably fibrant replacement of the split extension $H \mathbb{Z} \times\left(B \otimes \mathcal{S}^{k}\right)^{!}$ which was chosen in the beginning is equal to $H \mathbb{Z} \times\left(B \otimes \mathcal{S}^{k}\right)_{s t}^{!}$. 
The simplicial group $U\left(H \mathbb{Z} \times\left(B \otimes \mathcal{S}^{k}\right)^{!}\right)^{\times}$is defined according to the recipe (4.2) by a factorization in the model category of simplicial monoids

$$
B \longrightarrow c\left(H \mathbb{Z} \times\left(B \otimes \mathcal{S}^{k}\right)^{!}\right)^{\times} \stackrel{\sim}{\longrightarrow}\left(H \mathbb{Z} \times\left(B \otimes \mathcal{S}^{k}\right)^{!}\right)^{\times}
$$

and then forming the algebraic group completion of $c\left(H \mathbb{Z} \times\left(B \otimes \mathcal{S}^{k}\right)^{!}\right)^{\times}$. The simplicial monoid $\left(H \mathbb{Z} \times\left(B \otimes \mathcal{S}^{k}\right)^{!}\right)^{\times}$is, by definition, the union of the invertible components in $\left(H \mathbb{Z} \times\left(B \otimes \mathcal{S}^{k}\right)_{s t}^{!}\right)\left(1^{+}\right) \cong \mathbb{Z} \times\left(B \otimes \mathcal{S}^{k}\right)_{s t}(\mathbb{Z})$. So the simplicial monoid

$$
\left(H \mathbb{Z} \times\left(B \otimes \mathcal{S}^{k}\right)_{s t}^{!}\right)^{\times} \cong\{ \pm 1\} \times\left(B \otimes \mathcal{S}^{k}\right)_{s t}(\mathbb{Z})
$$

is already a simplicial group, hence there exists a unique extension to a homomorphism of simplicial groups $U\left(H \mathbb{Z} \times\left(B \otimes \mathcal{S}^{k}\right)^{!}\right)^{\times} \longrightarrow\{ \pm 1\} \times\left(B \otimes \mathcal{S}^{k}\right)_{s t}(\mathbb{Z})$ which is necessarily a weak equivalence by Lemma 4.3

Since $k \geq 2$, the stable derived functors of $B \otimes \mathcal{S}^{k}$ vanish in dimension 0 and 1 [11, 12.3], so the simplicial abelian group $\left(B \otimes \mathcal{S}^{k}\right)_{s t}(\mathbb{Z})$ is simply connected, and the unit component of the monoid $\left(H \mathbb{Z} \times\left(B \otimes \mathcal{S}^{k}\right)_{s t}^{!}\right)\left(1^{+}\right)$is equal to $\left(B \otimes \mathcal{S}^{k}\right)_{s t}(\mathbb{Z})$. Restriction to the unit components thus gives a weak equivalence of connected simplicial groups $U\left(H \mathbb{Z} \times\left(B \otimes \mathcal{S}^{k}\right)^{!}\right)_{1}^{\times} \longrightarrow\left(B \otimes \mathcal{S}^{k}\right)_{s t}(\mathbb{Z})$.

The next step in the construction of the conjugation action (4.2) was the formation of the pushout $\left(H \mathbb{Z} \times\left(B \otimes \mathcal{S}^{k}\right)^{!}\right)_{2}$ of Gamma-rings:

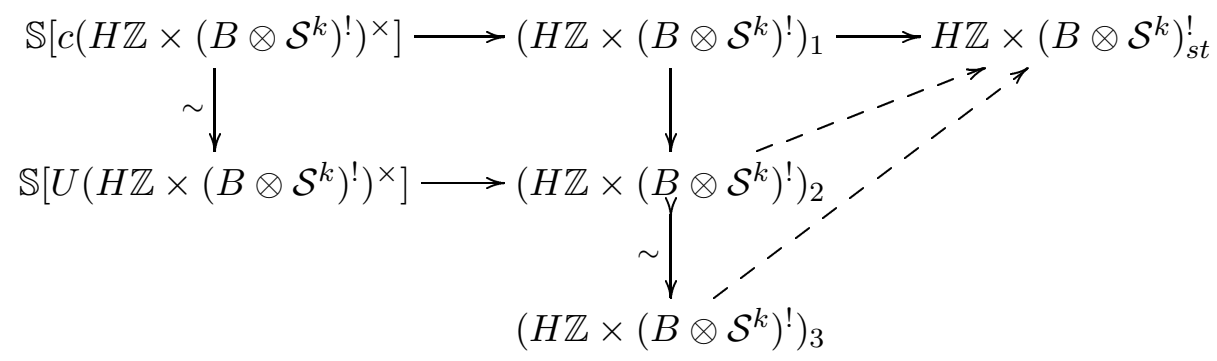

The simplicial group map $U\left(H \mathbb{Z} \times\left(B \otimes \mathcal{S}^{k}\right)^{!}\right)^{\times} \longrightarrow\{ \pm 1\} \times\left(B \otimes \mathcal{S}^{k}\right)_{\text {st }}(\mathbb{Z})$ adjoins to a homomorphism of Gamma-rings from the spherical group ring $\mathbb{S}\left[U\left(H \mathbb{Z} \times\left(B \otimes \mathcal{S}^{k}\right)^{!}\right)^{\times}\right]$to $H \mathbb{Z} \times\left(B \otimes \mathcal{S}^{k}\right)_{s t}^{!}$. This in turn induces a map from the pushout $\left(H \mathbb{Z} \times\left(B \otimes \mathcal{S}^{k}\right)^{!}\right)_{2}$ to $H \mathbb{Z} \times\left(B \otimes \mathcal{S}^{k}\right)_{\text {st }}^{!}$, represented as the upper dotted arrow in the previous diagram. Since the approximation map $\left(H \mathbb{Z} \times\left(B \otimes \mathcal{S}^{k}\right)^{!}\right)_{2} \longrightarrow\left(H \mathbb{Z} \times\left(B \otimes \mathcal{S}^{k}\right)^{!}\right)_{3}$ is an acyclic cofibration of Gamma-rings, we can finally choose an extension to a stable equivalence from $\left(H \mathbb{Z} \times\left(B \otimes \mathcal{S}^{k}\right)^{!}\right)_{3}$ to $H \mathbb{Z} \times\left(B \otimes \mathcal{S}^{k}\right)_{s t}^{!}$. Since this map was constructed relative to the group ring of the simplicial group $U\left(H \mathbb{Z} \times\left(B \otimes \mathcal{S}^{k}\right)^{!}\right)^{\times}$, it is equivariant with respect to the conjugation action of $U\left(H \mathbb{Z} \times\left(B \otimes \mathcal{S}^{k}\right)^{!}\right)_{1}^{\times}$on 
$\left(H \mathbb{Z} \times\left(B \otimes \mathcal{S}^{k}\right)^{!}\right)_{3}$ and (through the map $\left.U\left(H \mathbb{Z} \times\left(B \otimes \mathcal{S}^{k}\right)^{!}\right)_{1}^{\times} \longrightarrow\left(B \otimes \mathcal{S}^{k}\right)_{s t}(\mathbb{Z})\right)$ on $H \mathbb{Z} \times\left(B \otimes \mathcal{S}^{k}\right)_{s t}^{!}$. By passage to homotopy orbits we get a weak equivalence

$$
\begin{aligned}
\operatorname{der}\left(H \mathbb{Z}, B \otimes \mathcal{S}^{k}\right) / \text { conj. }= & \operatorname{map}_{\mathcal{G R}}\left(H \mathbb{Z}^{\mathrm{c}},\left(H \mathbb{Z} \times\left(B \otimes \mathcal{S}^{k}\right)^{!}\right)_{3}\right)_{h U\left(H \mathbb{Z} \times\left(B \otimes \mathcal{S}^{k}\right)^{!}\right)_{1}^{\times}} \\
& \left.\sim \operatorname{map}_{\mathcal{G R}}\left(H \mathbb{Z}^{\mathrm{c}}, H \mathbb{Z} \times\left(B \otimes \mathcal{S}^{k}\right)_{s t}\right)_{h\left(B \otimes \mathcal{S}^{k}\right.}\right)_{s t}(\mathbb{Z})
\end{aligned}
$$

Moreover, the square (9.1) commutes.

\section{A useful adjunction}

By the results of the previous two sections, the proof of the main theorem is reduced to an identification of the space of Gamma-ring maps

$$
\operatorname{map}_{\mathcal{G R}}\left(H \mathbb{Z}^{\mathrm{c}}, H \mathbb{Z} \times\left(B \otimes \mathcal{S}^{k}\right)_{s t}^{!}\right)
$$

(or more precisely a certain homotopy orbit space thereof) with the classifying space of symmetric 2-cocycles. In this section we use an adjunction to reinterpret the above mapping space in terms of the category $s \mathcal{F}$ of simplicial functors from the category of finitely generated free abelian groups to the category of abelian groups.

We note in Lemma10.4 that the construction (7.4) of the split singular extension has a left adjoint

$$
\mathcal{L}: \mathcal{G} R / H \mathbb{Z} \longrightarrow s \mathcal{F}
$$

from the category of Gamma-rings over $H \mathbb{Z}$ to the category of simplicial functors. Moreover, the two functors form a simplicial Quillen adjoint pair of model categories. So the mapping space we are interested in is isomorphic to the mapping space

$$
\operatorname{map}_{s \mathcal{F}}\left(\mathcal{L}\left(H \mathbb{Z}^{\mathrm{c}}\right),\left(B \otimes \mathcal{S}^{k}\right)_{s t}\right)
$$

in the category of simplicial functors. To identify the mapping space in this adjoint form, we evaluate the left adjoint $\mathcal{L}$ on the cofibrant replacement of the Eilenberg-MacLane Gamma-ring $H \mathbb{Z}$.

As in (8.3), $J \in \mathcal{F}$ denotes the kernel of the evaluation map $\epsilon: P \longrightarrow I$. By Lemma 8.5 the functor $J$ supports the universal symmetric 2-cocycle. The Gamma-ring map

$$
H \mathbb{Z}^{\mathrm{c}} \longrightarrow H \mathbb{Z} \times J^{!}
$$

which is the composite of the approximation map $H \mathbb{Z}^{\mathrm{c}} \longrightarrow H \mathbb{Z}$ and the universal derivation (8.6) is adjoint to a map of simplicial functors

$$
\delta: \mathcal{L}\left(H \mathbb{Z}^{\mathrm{c}}\right) \longrightarrow J .
$$

The main result of this section is 
Theorem 10.2 The map $\delta: \mathcal{L}\left(H \mathbb{Z}^{c}\right) \longrightarrow J$ which is adjoint to the universal derivation is a stable equivalence of simplicial functors.

Remark 10.3 For any functor $G \in \mathcal{F}$ the Dold-Puppe stabilization $G_{s t}$ was defined so that its normalized chain complex is the cubical construction $Q G$ (8.9). So Theorem 10.2 and the Dold-Kan correspondence between simplicial objects and chain complexes in the abelian category $\mathcal{F}$ imply that the homotopy groups of the space

$$
\operatorname{map}_{\mathcal{G R}}\left(H \mathbb{Z}^{\mathrm{c}}, H \mathbb{Z} \times G_{s t}^{!}\right) \cong \operatorname{map}_{s \mathcal{F}}\left(\mathcal{L}\left(H \mathbb{Z}^{\mathrm{c}}\right), G_{s t}\right)
$$

are isomorphic to the hyper-cohomology groups $\mathbb{E x t}_{\mathcal{F}}^{*}(J, Q G)$, for $* \leq 0$.

The category $s \mathcal{F}$ of simplicial functors admits a stable model structure, see 46, 6.13]. In this model structure, a map $G \longrightarrow \bar{G}$ is a weak equivalence or fibration if and only if the associated map of $\Gamma$-spaces $G^{!} \longrightarrow \bar{G}^{!}$is a stable equivalence or stable fibration respectively. The stably fibrant objects are precisely the homotopy additive simplicial functors.

The split extension functor (7.4) which sends $G \in s \mathcal{F}$ to $H \mathbb{Z} \times G^{!}$, considered as a Gamma-ring over $H \mathbb{Z}$, commutes with limits. Moreover, the category $s \mathcal{F}$ of simplicial functors is complete, well-powered and has a set of cogenerators. So by Freyd's Adjoint Functor Theorem [31, V.8 Cor.] there is a left adjoint $\mathcal{L}: \mathcal{G} R / H \mathbb{Z} \longrightarrow s \mathcal{F}$. The right adjoint $H \mathbb{Z} \times(-)^{!}$preserves stable equivalences and stable fibrations since in both categories these are defined on "underlying" $\Gamma$-spaces. Hence we obtain

Lemma 10.4 The functor which sends a simplicial functor $G \in s \mathcal{F}$ to $H \mathbb{Z} \times$ $G^{!}$, viewed as a Gamma-ring over $H \mathbb{Z}$, is the right adjoint of a Quillen adjoint pair between the category $s \mathcal{F}$ of simplicial functors, endowed with the stable model structure, and the stable model category of Gamma-rings over $H \mathbb{Z}$.

We prove Theorem 10.2 by constructing a commutative square of $H \mathbb{Z}^{\mathrm{c}}$-bimodules

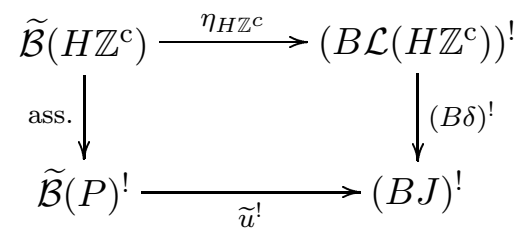

For a simplicial functor $G \in s \mathcal{F}$ we denote by $B G=\widetilde{\mathbb{Z}}\left[S^{1}\right] \otimes G$ the (additive) bar construction, another simplicial functor. The bimodules $\widetilde{\mathcal{B}}\left(H \mathbb{Z}^{\mathrm{c}}\right)$ and $\widetilde{\mathcal{B}}(P)^{\text {! }}$ 
are "multiplicative" bar constructions defined below. Three of the four objects are actually $H \mathbb{Z}$-bimodules, which we view as $H \mathbb{Z}^{\mathrm{c}}$-bimodules via restriction of scalars. In the square the left vertical and the two horizontal maps are stable equivalences by Theorems 10.7 10.9 and 10.10 respectively. Hence the map $(B \delta)^{!}:\left(B \mathcal{L}\left(H \mathbb{Z}^{\mathrm{c}}\right)\right)^{!} \longrightarrow(B J)^{!}$is a stable equivalence. Since the assignment $G \longmapsto(B G)^{!}$detects stable equivalences, the map $\delta: \mathcal{L}\left(H \mathbb{Z}^{\mathrm{c}}\right) \longrightarrow J$ is indeed a stable equivalence of simplicial functors.

10.6 A bar construction The reduced bar construction is a functor

$$
\widetilde{\mathcal{B}}: \mathcal{G} \mathcal{R} / H \mathbb{Z}^{\mathrm{c}} \longrightarrow H \mathbb{Z}^{\mathrm{c}}-\bmod -H \mathbb{Z}^{\mathrm{c}}
$$

from the category of Gamma-rings over $H \mathbb{Z}^{\mathrm{c}}$ to the category of $H \mathbb{Z}^{\mathrm{c}}$-bimodules. In this construction it is important that we start with Gamma-rings over the cofibrant approximation $H \mathbb{Z}^{\mathrm{c}}$, not just over $H \mathbb{Z}$. If we worked over $H \mathbb{Z}$, the bar construction would have the wrong homotopy type since the point set level smash product of $H \mathbb{Z}$ with itself is not stably equivalent to $H \mathbb{Z}^{\mathrm{c}} \wedge H \mathbb{Z}^{\mathrm{c}}$

If $Q$ is a Gamma-ring over $H \mathbb{Z}^{\mathrm{c}}$, then the (unreduced) bar construction $\mathcal{B}(Q)$ is defined as the realization of a simplicial $H \mathbb{Z}^{\mathrm{c}}$-bimodule which in simplicial dimension $n$ has the form

$$
H \mathbb{Z}^{\mathrm{c}} \wedge \underbrace{Q \wedge \ldots \wedge Q}_{n} \wedge H \mathbb{Z}^{\mathrm{c}} .
$$

The simplicial structure maps are induced by the multiplication and unit map of $Q$ and the structure map $Q \longrightarrow H \mathbb{Z}^{\mathrm{c}}$. The inclusion of the 0-simplices induces a map

$$
H \mathbb{Z}^{\mathrm{c}} \wedge H \mathbb{Z}^{\mathrm{c}} \longrightarrow \mathcal{B}(Q)
$$

of $H \mathbb{Z}^{\mathrm{c}}$-bimodules, and the reduced bar construction $\widetilde{\mathcal{B}}(Q)$ is the quotient of $\mathcal{B}(Q)$ by $H \mathbb{Z}^{\mathrm{c}} \wedge H \mathbb{Z}^{\mathrm{c}}$.

For every simplicial functor $G$ there is a map

$$
\tau_{G}: \widetilde{\mathcal{B}}\left(H \mathbb{Z}^{\mathrm{c}} \times G^{!}\right) \longrightarrow(B G)^{!}
$$

defined as the geometric realization of a map of simplicial $H \mathbb{Z}^{\mathrm{c}}$-bimodules

$$
n \mapsto[H \mathbb{Z}^{\mathrm{c}} \wedge \underbrace{\left(H \mathbb{Z}^{\mathrm{c}} \times G^{!}\right) \wedge \ldots \wedge\left(H \mathbb{Z}^{\mathrm{c}} \times G^{!}\right)}_{n} \wedge H \mathbb{Z}^{\mathrm{c}} \longrightarrow \underbrace{G^{!} \times \cdots \times G^{!}}_{n}]
$$

whose $i$-th projection, for $1 \leq i \leq n$, is given symbolically by

$x_{0} \wedge\left(x_{1}, g_{1}\right) \wedge \ldots \wedge\left(x_{n}, g_{n}\right) \wedge x_{n+1} \mapsto x_{0} \cdot x_{1} \cdots x_{i-1} \cdot g_{i} \cdot x_{i+1} \cdots x_{n} \cdot x_{n+1}$. 
We define a map $\eta_{Q}: \widetilde{\mathcal{B}}(Q) \longrightarrow(B \mathcal{L} Q)^{!}$as the composite

$$
\widetilde{\mathcal{B}}(Q) \longrightarrow \widetilde{\mathcal{B}}\left(H \mathbb{Z}^{\mathrm{c}} \times(\mathcal{L} Q)^{!}\right) \stackrel{\tau_{\mathcal{L} Q}}{\longrightarrow}(B \mathcal{L} Q)^{!} ;
$$

the first map is induced by the Gamma-ring map $Q \longrightarrow H \mathbb{Z}^{\mathrm{c}} \times(\mathcal{L} Q)^{!}$, which in turn comes from the structure map $Q \longrightarrow H \mathbb{Z}^{\mathrm{c}}$ and the adjunction unit $Q \longrightarrow H \mathbb{Z} \times(\mathcal{L} Q)^{!}$. For the proof of Theorem 10.2 we are only interested in the special case $Q=H \mathbb{Z}^{\mathrm{c}}$, but to establish that $\eta_{H \mathbb{Z}^{c}}$ is a weak equivalence we will use a resolution argument which requires the general case of an arbitrary Gamma-ring over $H \mathbb{Z}^{\mathrm{c}}$.

Theorem 10.7 The map of $H \mathbb{Z}^{c}$-bimodules

$$
\eta_{Q}: \widetilde{\mathcal{B}}(Q) \longrightarrow(B \mathcal{L}(Q))^{!}
$$

is a stable equivalence for every cofibrant Gamma-ring $Q$ over $H \mathbb{Z}^{c}$.

Proof We first assume that $Q$ is free, ie, that

$$
Q=T X=\bigvee_{n \geq 0} X^{\wedge n}
$$

is the tensor algebra generated by a cofibrant $\Gamma$-space $X$ over $H \mathbb{Z}^{\mathrm{c}}$. Then the $H \mathbb{Z}^{\mathrm{c}}$-bimodule $\widetilde{\mathcal{B}}(T X)$ can be analyzed through a combinatorial filtration as follows. For $p \geq 0$ we define $F_{p} \mathcal{B}$ as the realization of a simplicial sub- $H \mathbb{Z}^{\mathrm{c}}-$ bimodule of the bar construction $\mathcal{B}(T X)$. In simplicial degree $n$ we set

$$
\begin{aligned}
\left(F_{p} \mathcal{B}\right)_{n} & =H \mathbb{Z}^{\mathrm{c}} \wedge\left(\bigvee_{i_{1}+\cdots+i_{n} \leq p} X^{\wedge i_{1}} \wedge \ldots \wedge X^{\wedge i_{n}}\right) \wedge H \mathbb{Z}^{\mathrm{c}} \\
& \subseteq H \mathbb{Z}^{\mathrm{c}} \wedge(T X)^{\wedge} \wedge H \mathbb{Z}^{\mathrm{c}}=\mathcal{B}(T X)_{n} .
\end{aligned}
$$

The 0 -th filtration is $H \mathbb{Z}^{\mathrm{c}} \wedge H \mathbb{Z}^{\mathrm{c}}$ and the subquotient $F_{1} \mathcal{B} / F_{0} \mathcal{B}$ is isomorphic to the suspension of $H \mathbb{Z}^{\mathrm{c}} \wedge X \wedge H \mathbb{Z}^{\mathrm{c}}$. To identify the subquotients of the filtration we use certain simplicial sets $D_{p}$. We define $D_{p}$ as the quotient of the standard simplicial $p$-simplex with the union of the first and last $(p-1)$ face collapsed to a basepoint. Then $D_{1}=S^{1}$ is the simplicial circle and $D_{p}$ is weakly contractible for $p \geq 2$.

We note that the $p$-th subquotient is the realization of a simplicial object which in dimension $n$ is of the form

$$
\left(F_{p} \mathcal{B} / F_{p-1} \mathcal{B}\right)_{n}=H \mathbb{Z}^{\mathrm{c}} \wedge\left(\bigvee_{i_{1}+\cdots+i_{n}=p} X^{\wedge i_{1}} \wedge \ldots \wedge X^{\wedge i_{n}}\right) \wedge H \mathbb{Z}^{\mathrm{c}}
$$

The map

$$
H \mathbb{Z}^{\mathrm{c}} \wedge X^{\wedge p} \wedge H \mathbb{Z}^{\mathrm{c}} \longrightarrow\left(F_{p} \mathcal{B} / F_{p-1} \mathcal{B}\right)_{p}
$$


indexed by the $p$-tuple $(1,1, \ldots, 1)$ yields a map

$$
H \mathbb{Z}^{\mathrm{c}} \wedge X^{\wedge p} \wedge H \mathbb{Z}^{\mathrm{c}} \wedge \Delta^{p} \longrightarrow F_{p} \mathcal{B} / F_{p-1} \mathcal{B}
$$

which factors over an isomorphism between $H \mathbb{Z}^{\mathrm{c}} \wedge X^{\wedge p} \wedge H \mathbb{Z}^{\mathrm{c}} \wedge D_{p}$ and $F_{p} \mathcal{B} / F_{p-1} \mathcal{B}$. Since $D_{p}$ is weakly contractible for $p \geq 2$, all the filtration subquotients $F_{p} \mathcal{B} / F_{p-1} \mathcal{B}$ are stably contractible for $p \geq 2$. So the inclusion

$$
H \mathbb{Z}^{\mathrm{c}} \wedge \Sigma X \wedge H \mathbb{Z}^{\mathrm{c}} \cong F_{1} \mathcal{B} / F_{0} \mathcal{B} \stackrel{i}{\longrightarrow} \mathcal{B}(T X) / F_{0} \mathcal{B}=\widetilde{\mathcal{B}}(T X)
$$

is a stable equivalence of $H \mathbb{Z}^{\mathrm{c}}$-bimodules. To complete the verification that $\eta_{T X}$ is a stable equivalence it remains to show that the composite

$$
\eta_{T X} \circ i: H \mathbb{Z}^{\mathrm{c}} \wedge \Sigma X \wedge H \mathbb{Z}^{\mathrm{c}} \longrightarrow(B \mathcal{L}(T X))^{!}
$$

is a stable equivalence.

We can rewrite the target $(B \mathcal{L}(T X))^{!}$in a more familiar form. Let $\Phi$ denote the forgetful functor from the category of finitely generated free abelian groups to the category of pointed sets, and let $\widetilde{\mathbb{Z}}$ denote the reduced free functor from the category of pointed sets to the category of all abelian groups. By composing the $\Gamma$-space $X$ with these two functor we obtain an object $\widetilde{\mathbb{Z}} \odot X \circ \Phi$ of the category $s \mathcal{F}$. The various adjunctions show that $\widetilde{\mathbb{Z}} \circ X \circ \Phi$ and $\mathcal{L}(T X)$ represent the same functor, namely the one which sends an object $G \in s \mathcal{F}$ to the set of $\Gamma$-space from $X$ to the underlying $\Gamma$-space of $G^{!}$. Hence $\mathcal{L}(T X)$ is isomorphic to $\widetilde{\mathbb{Z}} \circ X \circ \Phi$ in the category $s \mathcal{F}$. Since the free functor $\widetilde{\mathbb{Z}}$ takes suspension of simplicial sets to bar construction of simplicial abelian groups, $B \mathcal{L}(T X)$ is then isomorphic to $\widetilde{\mathbb{Z}} \circ \Sigma X \circ \Phi$ in the category $s \mathcal{F}$.

Under the isomorphism $B \mathcal{L}(T X) \cong \widetilde{\mathbb{Z}} \circ \Sigma X \circ \Phi$ the map $\eta_{T X} \circ i$ corresponds to the composite

$$
\begin{aligned}
H \mathbb{Z}^{\mathrm{c}} \wedge \Sigma X \wedge H \mathbb{Z}^{\mathrm{c}} \longrightarrow H \mathbb{Z}^{\mathrm{c}} \circ \Sigma X \circ H \mathbb{Z}^{\mathrm{c}} \longrightarrow H \mathbb{Z} \circ \Sigma X \circ H \mathbb{Z} \\
=(\widetilde{\mathbb{Z}} \odot \Sigma X \circ \Phi)^{!} \cong(B \mathcal{L}(T X))^{!} .
\end{aligned}
$$

The left map is the assembly map (2.5), which is a stable equivalence by 29, 5.23] since $H \mathbb{Z}^{\mathrm{c}}$ and $\Sigma X$ are cofibrant as $\Gamma$-spaces. The second map is a weak equivalence since the composition product of $\Gamma$-spaces preserves stable equivalences (Theorem 2.6 (a)).

The general case is proved by resolving an arbitrary cofibrant Gamma-ring by free Gamma-rings as follows. If $R$ is a simplicial object in the category of Gamma-rings, we denote by $|R|_{\mathcal{G} R}$ its geometric realization [22, VII 3.1] in the category of Gamma-rings, ie, the coend [31, IX.6]

$$
|R|_{\mathcal{G} R}=\int_{n \in \Delta} R_{n} \times_{\mathcal{G} R} \Delta^{n}
$$


here $(-) \times_{\mathcal{G} R} \Delta^{n}$ refers to the enrichment of the category of Gamma-rings over simplicial sets, which has to be distinguished from the objectwise smash product of the underlying $\Gamma$-space with $\Delta_{+}^{n}$.

Claim Let $R$ be a simplicial object in the category of Gamma-rings over $H \mathbb{Z}^{\mathrm{c}}$ such that for all $n \geq 0$ the map $\eta_{R_{n}}: \widetilde{\mathcal{B}}\left(R_{n}\right) \longrightarrow\left(B \mathcal{L}\left(R_{n}\right)\right)^{!}$is a stable equivalence. Then the map $\eta_{|R|_{\mathcal{G} R}}: \widetilde{\mathcal{B}}\left(|R|_{\mathcal{G} R}\right) \longrightarrow\left(B \mathcal{L}\left(|R|_{\mathcal{G} R}\right)\right)^{!}$is also a stable equivalence.

To prove the claim it suffices to show that the map is a stable equivalence of underlying $\Gamma$-spaces. We consider the commutative square



On the left the functors $\widetilde{\mathcal{B}}$ and $(B \mathcal{L}-)^{!}$are applied dimensionwise to the simplicial Gamma-ring $R$, and then we form the realization of the underlying simplicial $\Gamma$-space. The two horizontal maps are isomorphisms, so we may show that the left vertical map is a stable equivalence. A map of simplicial $\Gamma$-spaces which is dimensionwise a stable equivalence becomes a stable equivalence after realization. So the left vertical map in the above square is a stable equivalence of underlying $\Gamma$-spaces, which proves the claim.

We apply the claim to the cotriple resolution [31, VII.6] of a given cofibrant Gamma-ring $Q$ over $H \mathbb{Z}^{\mathrm{c}}$. The tensor algebra functor $T$ from the category of $\Gamma$-spaces to the category of Gamma-rings is left adjoint to the forgetful functor. The adjunction gives rise to a cotriple, hence to a simplicial Gamma-ring $R$ which in simplicial dimension $n$ consists of the Gamma-ring $R_{n}=T^{n+1} Q$. We claim that this simplicial Gamma-ring $R$ is cofibrant in the Reedy model structure ([40, Thm. A], [23, 5.2.5], [22, VII 2.1]). Indeed, the maps from the latching objects to the levels of the simplicial Gamma-ring $R$ are freely generated by a wedge summand inclusion of $\Gamma$-spaces whose cokernel is a wedge of smash powers of $Q$. Since $Q$ is cofibrant as a Gamma-ring, it is cofibrant as a $\Gamma$-space [47, 4,1 (3)], hence so are its smash powers. So the maps from the latching objects to the levels of the $R$ are cofibrations of Gamma-rings, ie, $R$ is Reedy cofibrant. In particular, for all $n \geq 0$ the underlying $\Gamma$-space of $T^{n} Q$ is cofibrant, and hence for $R_{n}=T\left(T^{n} Q\right)$ the map $\eta_{R_{n}}: \widetilde{\mathcal{B}}\left(R_{n}\right) \longrightarrow\left(B \mathcal{L}\left(R_{n}\right)\right)^{!}$ is a stable equivalence by the first part of this proof. By the claim, the map $\eta_{|R|_{\mathcal{G} R}}: \widetilde{\mathcal{B}}\left(|R|_{\mathcal{G} R}\right) \longrightarrow\left(B \mathcal{L}\left(|R|_{\mathcal{G} R}\right)\right)^{!}$is also a stable equivalence. 
The cotriple resolution comes with an augmentation $|R|_{\mathcal{G} R} \longrightarrow Q$. After forgetting the multiplication, the augmented simplicial $\Gamma$-space $|R|_{\mathcal{G} R} \longrightarrow Q$ has an extra degeneracy, so the augmentation map $|R|_{\mathcal{G} R} \longrightarrow Q$ is an objectwise equivalence of Gamma-rings [22, III 5.1]. Since $R$ is Reedy cofibrant, the realization $|R|_{\mathcal{G} R}$ is cofibrant [22, VII 3.6]. Since the functors $\widetilde{\mathcal{B}}$ and $(B \mathcal{L}-)^{!}$both preserve stable equivalences between cofibrant Gamma-rings, $\eta_{Q}$ is a stable equivalence as claimed.

10.8 Another bar construction The lower left hand corner of the square (10.5) arises from a simplicial functor $\widetilde{\mathcal{B}}(P) \in s \mathcal{F}$ which is another reduced bar construction. We note that the category $\mathcal{F}$ of reduced functors from finitely generated free abelian groups to abelian abelian groups has a monoidal composition product $\circ$ with unit the inclusion functor $I$; before composing two functors, one of them has to be left Kan extended [31, X.3] from finitely generated free to all abelian groups.

The functor $P \in \mathcal{F}$ is the composite of the forgetful functor from abelian groups to pointed sets with its adjoint free functor. Hence $P$ has the structure of a cotriple on the category of abelian groups. This cotriple gives rise to a simplicial object $\mathcal{B}(P)$, augmented over the functor $I$, which in simplicial dimension $n$ is given by

$$
\mathcal{B}(P)_{n}=\underbrace{P \circ \cdots \circ P}_{n+1}
$$

compare [31, VII.6]. The augmentation $\mathcal{B}(P)_{0}=P \longrightarrow I$ is given by the evaluation map $\epsilon$.

The $H \mathbb{Z}$-bimodule $\mathcal{B}(P)_{n}^{!}=\left(P^{\circ(n+1)}\right)^{!}$is equal to the $(n+2)$-fold composition product of the Eilenberg-MacLane $\Gamma$-space $H \mathbb{Z}$. So the assembly map (2.5) induces a map of simplicial $H \mathbb{Z}^{\mathrm{c}}$-bimodules

$$
\mathcal{B}\left(H \mathbb{Z}^{\mathrm{c}}\right)_{n}=\left(H \mathbb{Z}^{\mathrm{c}}\right)^{\wedge(n+2)} \stackrel{\text { assembly }}{\longrightarrow} H \mathbb{Z}^{\circ(n+2)}=\mathcal{B}(P)_{n}^{!} .
$$

We denote by $\widetilde{\mathcal{B}}(P)$ the simplicial functor obtained from $\mathcal{B}(P)$ by collapsing the simplicial 0-skeleton. The assembly map passes to a map $\widetilde{\mathcal{B}}\left(H \mathbb{Z}^{\mathrm{c}}\right) \longrightarrow \widetilde{\mathcal{B}}(P)^{\text {! }}$ on quotients by the respective simplicial 0 -skeleta.

Theorem 10.9 The assembly map

$$
\widetilde{\mathcal{B}}\left(H \mathbb{Z}^{c}\right) \longrightarrow \widetilde{\mathcal{B}}(P)^{!}
$$

is a stable equivalence of $H \mathbb{Z}^{c}$-bimodules. 
Proof Since $H \mathbb{Z}^{c}$ is cofibrant as a $\Gamma$-space, the assembly map from a smash power of a certain number of copies of $H \mathbb{Z}^{\mathrm{c}}$ to the composition power of the same number of copies of $H \mathbb{Z}$ is a stable equivalence by [29, 5.23] and Theorem 2.6 (a). A map of simplicial $\Gamma$-spaces which is dimensionwise a stable equivalence induces a stable equivalence on realizations. So the assembly maps

$$
\mathcal{B}\left(H \mathbb{Z}^{\mathrm{c}}\right) \longrightarrow \mathcal{B}(P)^{!} \quad \text { and } \quad \widetilde{\mathcal{B}}\left(H \mathbb{Z}^{\mathrm{c}}\right) \longrightarrow \widetilde{\mathcal{B}}(P)^{!}
$$

on realizations are stable equivalences of $H \mathbb{Z}^{\mathrm{c}}$-bimodules.

The lower horizontal map in the square (10.5) arises from an objectwise equivalence

$$
u: \mathcal{B}(P) \longrightarrow P \oplus_{J} E J
$$

of simplicial functors by passage to quotient and application of the $(-)^{!}-$construction. The target $P \oplus_{J} E J$ is the simplicial functor defined as the pushout of the diagram

$$
P \stackrel{\iota}{\longleftarrow} J \longrightarrow \widetilde{\mathbb{Z}}\left[\Delta^{1}\right] \otimes J
$$

The map $\iota: J \longrightarrow P$ is the inclusion and $J \longrightarrow \widetilde{\mathbb{Z}}\left[\Delta^{1}\right] \otimes J$ is induced by the inclusion of the non-basepoint vertex of $\Delta^{1}$.

We describe the map

$$
u_{n}: \mathcal{B}(P)_{n}=P^{\circ(n+1)} \longrightarrow\left(P \oplus_{J} E J\right)_{n}=P \times J^{n}
$$

in simplicial dimension $n$ by giving the components of the various factors of the target. The projection of $u_{n}$ to the first factor is the map

$$
\epsilon^{\circ n} \circ 1: P^{\circ(n+1)} \longrightarrow P \text {. }
$$

The projection of $u_{n}$ to the $i$-th factor of $J$, for $1 \leq i \leq n$ is the map

$$
\epsilon^{\circ(n-i)} \circ 1 \circ \epsilon^{\circ i}-\epsilon^{\circ(n-i+1)} \circ 1 \circ \epsilon^{\circ(i-1)}: P^{\circ(n+1)} \longrightarrow J \text {; }
$$

the target of each of the two summands is really the functor $P$, but the difference is annihilated by $\epsilon: P \longrightarrow I$, so it lands in $J=\operatorname{kernel}(\epsilon)$. Note that $P$ is the functor of 0 -simplices in $P \oplus_{J} E J$, and the quotient of $P \oplus_{J} E J$ by $P$ is isomorphic to the simplicial functor $B J$. So the map $u$ passes to quotients and yields a map of simplicial functors $\widetilde{u}: \widetilde{\mathcal{B}}(P) \longrightarrow B J$.

Theorem 10.10 The map

$$
\widetilde{u}: \widetilde{\mathcal{B}}(P) \longrightarrow B J
$$

is an objectwise weak equivalence of simplicial functors. 
Proof The simplicial subfunctor $\widetilde{\mathbb{Z}}\left[\Delta^{1}\right] \otimes J$ of $P \oplus_{J} E J$ is objectwise weakly contractible. So the quotient map

$$
q: P \oplus_{J} E J \longrightarrow\left(P \oplus_{J} E J\right) /\left(\widetilde{\mathbb{Z}}\left[\Delta^{1}\right] \otimes J\right) \cong I
$$

is an objectwise weak equivalence of simplicial functors.

The composite map $q \circ u: \mathcal{B}(P) \longrightarrow I$ is the augmentation of the cotriple resolution. Whether or not it is an objectwise weak equivalence can be checked by looking at the augmented simplicial $\Gamma$-space $\mathcal{B}(P)^{!} \longrightarrow I^{!}$. However this augmented simplicial $\Gamma$-space has an extra degeneracy, so the augmentation $\mathcal{B}(P)^{!} \longrightarrow I^{!}$, and hence the map $u$, is an objectwise weak equivalence [22, III 5.1].

The simplicial 0-skeleta of $\mathcal{B}(P)$ and $P \oplus_{J} E J$ are both equal to the functor $P$. So if we collapse the 0 -skeleta, then the induced map of quotients $\widetilde{u}: \widetilde{\mathcal{B}}(P) \longrightarrow$ $B J$ is also an objectwise weak equivalence.

\section{A homological criterion}

In this section we give a homological condition, Theorem 11.1 below, for when the map

$$
\lambda_{G}: \mathcal{Z}(G) \longrightarrow \operatorname{map}_{\mathcal{G R}}\left(H \mathbb{Z}^{\mathrm{c}}, H \mathbb{Z} \times G_{s t}^{!}\right)_{h G_{s t}(\mathbb{Z})}
$$

defined in (8.10) is a weak equivalence. Here $\mathcal{Z}(G)$ denotes the groupoid of symmetric 2-cocycles of the functor $G$ (8.1). $G_{s t}$ is the Dold-Puppe stabilization of $G$, a simplicial functor which corresponds to the cubical construction $Q G$ under the Dold-Kan equivalence between simplicial objects and non-negative complexes in the category $\mathcal{F}$, compare (8.9). In the next section we verify the criterion of Theorem 11.1 in the case of the symmetric power functors $B \otimes \mathcal{S}^{k}$.

As before, $\mathcal{F}$ denotes the abelian category of reduced functors from finitely generated free abelian groups to all abelian groups and $I \in \mathcal{F}$ is the inclusion functor. The functor category $\mathcal{F}$ is abelian and exactness can be checked objectwise; $\mathcal{F}$ has enough projectives and injectives. The map $G \longrightarrow Q G$ is the inclusion as the object in dimension zero. Also, $\mathbb{E x t}_{\mathcal{F}}^{*}(I,-)$ denotes hyper-Ext groups of the inclusion functor $I$ with coefficients in a chain complex of functors, ie, the graded abelian group of maps out of $I$ in the derived category $\mathcal{D}^{+}(\mathcal{F})$ of bounded below complexes of functors. A priori, these hyper-Ext groups can be non-trivial in negative dimensions. 
Theorem 11.1 Let $G \in \mathcal{F}$ be a functor such that for all integers $m \leq 2$ the map

$$
\operatorname{Ext}_{\mathcal{F}}^{m}(I, G) \longrightarrow \mathbb{E x t}_{\mathcal{F}}^{m}(I, Q G)
$$

is an isomorphism. Then the map

$$
\lambda_{G}: \mathcal{Z}(G) \longrightarrow \operatorname{map}_{\mathcal{G R}}\left(H \mathbb{Z}^{c}, H \mathbb{Z} \times G_{s t}^{!}\right)_{h G_{s t}(\mathbb{Z})}
$$

is a weak equivalence of simplicial sets.

Remark 11.2 By [46, 6.1], the hyper-cohomology groups $\mathbb{E x t}_{\mathcal{F}}^{m}(I, Q G)$ are isomorphic to the topological Hochschild cohomology groups of $H \mathbb{Z}$ with coefficients in the bimodule $G^{!}$.

On the other hand, if $A$ is an abelian group, then a theorem of Jibladze and Pirashvili [26, Thm. A] identifies the cohomology groups $\operatorname{Ext}_{\mathcal{F}}^{*}(I, A \otimes-)$ with the MacLane cohomology groups of $A$ 30. Because of this, for an arbitrary functor $G \in \mathcal{F}$ the groups $\operatorname{Ext}_{\mathcal{F}}^{*}(I, G)$ are sometimes referred to as the MacLane cohomology groups of $\mathbb{Z}$ with coefficients in the functor $G$. So the criterion of Theorem 11.1 ask whether the natural map from the MacLane cohomology to the topological Hochschild cohomology of the functor $G$ is an isomorphism.

A theorem of Pirashvili and Waldhausen [37, 3.2] says that if cohomology is replaced by homology, then the MacLane theory coincides with the topological Hochschild theory for arbitrary coefficient functors. By [46, 6.7], the cohomological theories also agree if the coefficient functor is additive.

However, as the following example shows, the hypothesis of Theorem 11.1 is not satisfied for an arbitrary functor $G \in \mathcal{F}$. So for general coefficients, MacLane cohomology and topological Hochschild cohomology do not coincide.

Example 11.3 We give an example of a functor for which the hypothesis of Theorem 11.1 fails. For a fixed prime $p$ the Frobenius maps

$$
\mathbb{Z} / p \otimes \mathcal{S}^{p^{h-1}} \longrightarrow \mathbb{Z} / p \otimes \mathcal{S}^{p^{h}}, \quad a \otimes x \longmapsto a \otimes x^{p}
$$

define a morphism in the category $\mathcal{F}$. We consider the functor

$$
G=\operatorname{colim}_{h} \mathbb{Z} / p \otimes \mathcal{S}^{p^{h}}
$$

defined as the colimit of the sequence of Frobenius maps. Since the Frobenius transformations are injective, the natural map

$$
I \stackrel{\text { projection }}{\longrightarrow} \mathbb{Z} / p \otimes I=\mathbb{Z} / p \otimes \mathcal{S}^{p^{0}} \stackrel{\text { inclusion }}{\longrightarrow} G
$$

is a non-trivial element of $\operatorname{Hom}_{\mathcal{F}}(I, G)$. On the other hand, the stable derived functors of the symmetric power functor $A \otimes \mathcal{S}^{k}$ are trivial up to dimension $2 k-3$ 
[1], 12.3], so $Q\left(\mathbb{Z} / p \otimes \mathcal{S}^{p^{h}}\right)$ has trivial homology up to dimension $\left(2 p^{h}-3\right)$. Since the $Q$-construction and homology commute with filtered colimits, the complex $Q G$ is acyclic, so the hyper-cohomology groups $\mathbb{E x t}_{\mathcal{F}}^{*}(I, Q G)$ are trivial. In particular the map

$$
\operatorname{Ext}_{\mathcal{F}}^{0}(I, G) \longrightarrow \mathbb{E x t}_{\mathcal{F}}^{0}(I, Q G)
$$

is not injective.

Proof of Theorem 11.1 The map $\lambda_{G}$ (8.10) is obtained from the commutative square of simplicial abelian groups

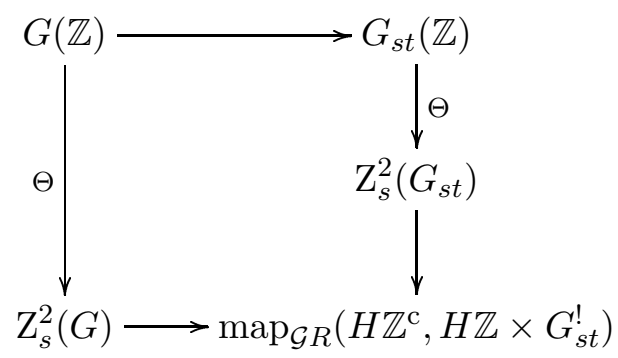

by passage to vertical homotopy cofibres in the category of simplicial abelian groups. So it suffices to show that the square is homotopy cocartesian (in the category of simplicial abelian groups). Evaluation at $\mathbb{Z}$ is represented by the projective functor $P$, symmetric $2-$ cocycles are represented by the functor $J$ (Lemma 8.5), and the split extension construction (7.4) has a left adjoint $\mathcal{L}$ (Lemma 10.4). So the square is isomorphic to the square

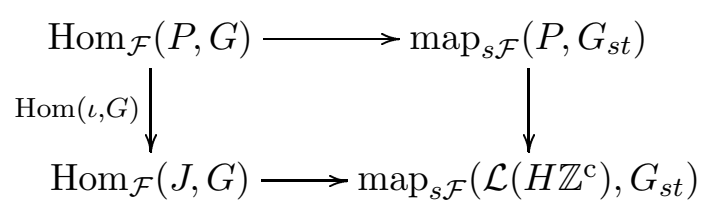

where $\iota: J \longrightarrow P$ is the inclusion.

The map $\delta: \mathcal{L}\left(H \mathbb{Z}^{\mathrm{c}}\right) \longrightarrow J$ (10.1) which is adjoint to the universal derivation is a stable equivalence by Theorem 10.2. We let $\alpha: J^{\mathrm{c}} \longrightarrow J$ be a cofibrant approximation of the functor $J$ in the strict model structure of simplicial functors where the weak equivalences are defined objectwise; equivalently, the normalized chain complex of $J^{\mathrm{c}}$ is a projective resolution of $J$. Then $\delta: \mathcal{L}\left(H \mathbb{Z}^{\mathrm{c}}\right) \longrightarrow J$ lifts to a map of simplicial functors $\bar{\delta}: \mathcal{L}\left(H \mathbb{Z}^{\mathrm{c}}\right) \longrightarrow J^{\mathrm{c}}$. The lift $\bar{\delta}$ is then a stable equivalence between cofibrant simplicial functors. Since $G_{s t}$ is homotopy additive, alias stably fibrant, the map $\bar{\delta}$ induces a weak equivalence of simplicial abelian groups upon application of $\operatorname{map}_{s \mathcal{F}}\left(-, G_{s t}\right)$. Since $G$ is a constant 
simplicial functor, the map

$$
\operatorname{map}_{s \mathcal{F}}(\alpha, G): \operatorname{Hom}_{\mathcal{F}}(J, G)=\operatorname{map}_{s \mathcal{F}}(J, G) \longrightarrow \operatorname{map}_{s \mathcal{F}}\left(J^{\mathrm{c}}, G\right)
$$

is an isomorphism. In other words, it suffices to show that the square in the category of simplicial abelian groups

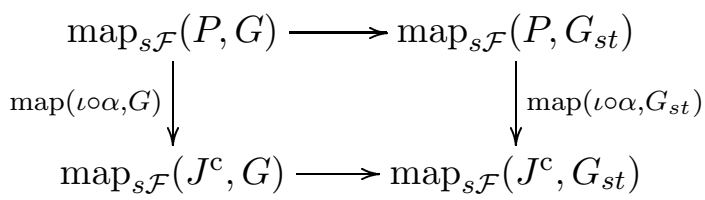

is homotopy cocartesian. For this in turn it is enough to show that the map on horizontal homotopy cofibres

$$
\operatorname{map}_{s \mathcal{F}}\left(\iota \circ \alpha, G_{s t} / G\right): \operatorname{map}_{s \mathcal{F}}\left(P, G_{s t} / G\right) \longrightarrow \operatorname{map}_{s \mathcal{F}}\left(J^{\mathrm{c}}, G_{s t} / G\right)
$$

is a weak equivalence where $G_{s t} / G$ denotes to cofibre of the stabilization map.

If $K$ and $K^{\prime}$ are two simplicial functors such that $K$ is cofibrant, then the Dold-Kan theorem provides a natural isomorphisms of groups

$$
\pi_{n} \operatorname{map}_{s \mathcal{F}}\left(K, K^{\prime}\right) \cong\left[N K[n], N K^{\prime}\right]
$$

for $n \geq 0$, where $N$ is the normalized chain complex and $[-,-]$ denotes maps in the derived category $\mathcal{D}^{+}(\mathcal{F})$ of bounded below chain complexes of functors. The normalized chain complex of $J^{\mathrm{c}}$ is quasi-isomorphic to $J$, and the normalized chain complex of $G_{s t}$ is the cubical construction $Q G$. So we need to show that the map

$$
[P[n], Q G / G] \longrightarrow[J[n], Q G / G]
$$

is an isomorphism for $n \geq 0$. The short exact sequence of functors $J \longrightarrow$ $P \longrightarrow I$ yields a long exact sequence after applying $[-, Q G / G]$, so it is enough to show that the groups

$$
[I[n], Q G / G]=\operatorname{Ext}_{\mathcal{F}}^{-n}(I, Q G / G)
$$

vanish for $n \geq-1$. This in turn follows from the assumption that the map

$$
\operatorname{Ext}_{\mathcal{F}}^{m}(I, G) \longrightarrow \operatorname{Ext}_{\mathcal{F}}^{m}(I, Q G)
$$

is an isomorphism for all integers $m \leq 2$.

\section{Cohomology of symmetric power functors}

The purpose of this section is to prove that the symmetric power functors satisfy the homological criterion of Theorem 11.1 this completes the proof of the main theorem. 
Theorem 12.1 For all $m \in \mathbb{Z}$, all $k \geq 1$ and all abelian groups $A$ the map

$$
\operatorname{Ext}_{\mathcal{F}}^{m}\left(I, A \otimes \mathcal{S}^{k}\right) \longrightarrow \mathbb{E x t}_{\mathcal{F}}^{m}\left(I, Q\left(A \otimes \mathcal{S}^{k}\right)\right)
$$

is an isomorphism.

Remark 12.2 Theorem 12.1 can be interpreted as saying that MacLane cohomology coincides with topological Hochschild cohomology for the symmetric power functors, compare Remark 11.2. The groups $\operatorname{Ext}_{\mathcal{F}}^{m}\left(I, A \otimes \mathcal{S}^{k}\right)$ have been calculated for $A=\mathbb{Z} / p$ and for $A=\mathbb{Z}$ by Franjou-Lannes-Schwartz and Franjou-Pirashvili, see [17, Thm. 6.6 and Prop. 9.1] and [18, 2.1]. So Theorem 12.1 is a calculation of the topological Hochschild cohomology groups of $H \mathbb{Z}$ with coefficients in the bimodule $\left(A \otimes \mathcal{S}^{k}\right)$ !. In particular, the topological Hochschild cohomology groups of $H \mathbb{Z}$ with coefficients in $\left(A \otimes \mathcal{S}^{k}\right)^{!}$are trivial in negative dimensions.

Our proof of Theorem 12.1 is not completely satisfactory because it uses the explicit calculations of the groups $\operatorname{Ext}_{\mathcal{F}}^{m}\left(I, \mathcal{S}^{k}\right)$; these enter as the sparseness hypothesis (c) of Theorem 12.3 below. It would be desirable to have a direct proof of Theorem 12.1 which would hopefully shed more light on the question for which coefficient functors MacLane cohomology coincides with topological Hochschild cohomology. Example 11.3 shows that some restriction on the functor has to be imposed.

Theorem 12.1 is a special case of the following Theorem 12.3 To apply it, we choose a projective resolution $P_{*} \longrightarrow I$ of the functor $I$ in the abelian category $\mathcal{F}$. Then we let $T: \mathrm{Ch}^{+}(\mathcal{F}) \longrightarrow$ coCh be the homomorphism complex out of this resolution,

$$
T(X)=\operatorname{Hom}_{\mathcal{F}}^{\bullet}\left(P_{*}, X\right) .
$$

So $T(X)$ is a (usually unbounded) cochain complex of abelian groups and as a functor of $X$ it is additive, exact, and preserves inverse limits and quasiisomorphisms. The cohomology groups of $\operatorname{Hom}_{\mathcal{F}}^{\bullet}\left(P_{*}, X\right)$ are the hyper-cohomology groups $\mathbb{E x t}_{\mathcal{F}}^{*}(I, X)$. By a theorem of Pirashvili ([34, 2.15], see also [17. 0.4] or the appendix of [4]), the extension groups $\operatorname{Ext}_{\mathcal{F}}^{*}(I,-)$ vanish for every diagonalizable functor $8.9(\mathrm{e}))$, so $\operatorname{Hom}_{\mathcal{F}}^{\bullet}\left(P_{*},-\right)$ takes diagonalizable functors to acyclic complexes. The sparseness condition (c) is proved in 18 , Prop. 2.1].

The homomorphism complex $\operatorname{Hom}_{\mathcal{F}}\left(P_{*},-\right)$ is the only functor to which we apply Theorem 12.3 nevertheless we state and prove it in the general form because we think it makes the proof more understandable. 
Theorem 12.3 Let $T: \mathrm{Ch}^{+}(\mathcal{F}) \longrightarrow$ coCh be a functor from the category of bounded below chain complexes in the abelian category $\mathcal{F}$ to the category of (not necessarily bounded) cochain complexes of abelian groups. Suppose furthermore that

(a) $T$ is additive, exact and preserves inverse limits and quasi-isomorphisms,

(b) the complex $T(D)$ is acyclic for every diagonalizable functor $D \in \mathcal{F} 8.9$ (e)), considered as a complex concentrated in dimension 0 , and

(c) $T$ is sparse on symmetric powers in the sense that for all $k \geq 1$ the cohomology of the complex $T\left(\mathcal{S}^{k}\right)$ is concentrated in dimensions congruent to 1 modulo $2 k$.

Then for all abelian groups $A$, and all $k \geq 1$ the natural map

$$
T\left(A \otimes \mathcal{S}^{k}\right) \longrightarrow T\left(Q\left(A \otimes \mathcal{S}^{k}\right)\right)
$$

is a quasi-isomorphism.

Remark 12.4 The heart of Theorem 12.3 is a convergence issue, or a question to what extent the functor $T$ commutes with infinite sums (up to quasiisomorphism). Indeed, for any functor $G \in \mathcal{F}$, the cokernel of the stabilization map $G \longrightarrow Q G$ is a bounded below complex of diagonalizable functors [27, 7.4], usually with non-trivial homology in arbitrarily high dimensions. So the cokernel $Q G / G$ can be written as the colimit of a sequence of bounded complex of diagonalizable functors. So if a functor $T$ as in Theorem 12.3 commutes with filtered colimits or infinite sums, then properties (a) and (b) already imply that $T(Q G / G)$ is acyclic, and so $T(G) \longrightarrow T(Q G)$ is a quasi-isomorphism, for all $G \in \mathcal{F}$. In the case of interest for us, namely $T=\operatorname{Hom}_{\mathcal{F}}^{\bullet}\left(P_{*},-\right)$, the functor $T$ fails to commute with infinite sums, essentially because the inclusion functor $I$ is not a small (or compact) object in the derived category of $\mathcal{F}$. And indeed, $\operatorname{Hom}_{\mathcal{F}}^{\bullet}\left(P_{*}, Q G / G\right)$ fails to be acyclic in general as Example 11.3 shows. However the sparseness condition (c) makes it possible to obtain the desired conclusion for the symmetric power functors.

The following observation goes back, at least, to Dold and Puppe [1]. For every $k \geq 1$ let $d_{k}$ denote the greatest common divisor of the binomial coefficients $\left(\begin{array}{c}k \\ i\end{array}\right)$ for $1 \leq i \leq k-1$. Then

$$
d_{k}= \begin{cases}p & \text { if } k=p^{h} \text { for a prime } p \text { and } h>0 \\ 1 & \text { else. }\end{cases}
$$


Lemma 12.5 11, 10.9] For every $k \geq 1$ and every abelian group $A$, multiplication by the number $d_{k}$ on the functor $A \otimes \mathcal{S}^{k}$ factors over a diagonalizable functor 8.9 (e)). In particular, if $k$ is not a prime power, then $A \otimes \mathcal{S}^{k}$ is a retract of a diagonalizable functor.

Proof For every $1 \leq i \leq k-1$ the comultiplication of the symmetric algebra gives a map of functors

$$
\Delta_{i, k-i}: A \otimes \mathcal{S}^{k} \longrightarrow A \otimes \mathcal{S}^{i} \otimes \mathcal{S}^{k-i} ;
$$

the explicit formula for this map is given by

$$
a \otimes x_{1} \cdots x_{k} \longmapsto a \otimes \sum_{T \subset\{1,2, \ldots, k\},|T|=i}\left(\prod_{j \in T} x_{j}\right) \otimes\left(\prod_{j \notin T} x_{j}\right) .
$$

The composite of $\Delta_{i, k-i}$ with the natural projection $A \otimes \mathcal{S}^{i} \otimes \mathcal{S}^{k-i} \longrightarrow A \otimes \mathcal{S}^{k}$ is multiplication by the binomial coefficient $\left(\begin{array}{l}k \\ i\end{array}\right)$. So if we choose a presentation

$$
d_{k}=\sum_{i=1}^{k-1} \lambda_{i} \cdot\left(\begin{array}{l}
k \\
i
\end{array}\right)
$$

for suitable integers $\lambda_{i}$ then the composition

$$
A \otimes \mathcal{S}^{k} \stackrel{\sum \lambda_{i} \cdot \Delta_{i, k-i}}{\longrightarrow} \bigoplus_{i=1}^{k-1} A \otimes \mathcal{S}^{i} \otimes \mathcal{S}^{k-i} \longrightarrow A \otimes \mathcal{S}^{k}
$$

is multiplication by the number $d_{k}$.

Finally, we give the proof of Theorem 12.3 For a functor $G \in \mathcal{F}$ we use the notation $\bar{Q} G$ for the quotient complex $Q G / G$. By the exactness of $T$ we then have to show that for all abelian groups $A$, and all $k \geq 1$ the complex $T\left(\bar{Q}\left(A \otimes \mathcal{S}^{k}\right)\right)$ is acyclic.

\section{Step 1 Diagonalizable functors}

Suppose $D \in \mathcal{F}$ is diagonalizable 8.9 (e)). Then $Q D$ is an acyclic complex by 8.9 (e), and so $T(Q D)$ is acyclic. By property (b) of the functor $T$ the complex $T(D)$, and hence, by exactness, the quotient complex $T(\bar{Q} D)$ is also acyclic.

If $k$ is not a prime power then $A \otimes \mathcal{S}^{k}$ is a retract of a diagonalizable functor by Lemma 12.5. so Theorem 12.3 holds for such exponents. From now on we assume that the exponent is of the form $k=p^{h}$ for a prime number $p$ and some $h \geq 0$. 


\section{Step 2 Reduction to the case $A=\mathbb{Z} / p$}

For the course of this proof we call an abelian group $A$ good if the complex

$$
T\left(\bar{Q}\left(A \otimes \mathcal{S}^{p^{h}}\right)\right)
$$

is acyclic. We show that if the group $\mathbb{Z} / p$ is good, then every abelian group is good.

Multiplication by the number $p$ is an epimorphism on the functor $\mathbb{Q} / \mathbb{Z} \otimes \mathcal{S}^{p^{h}}$ with kernel isomorphic to $\mathbb{Z} / p \otimes \mathcal{S}^{p^{h}}$. Since the cubical construction and the functor $T$ are exact, multiplication by $p$ on the complex $T\left(\bar{Q}\left(\mathbb{Q} / \mathbb{Z} \otimes \mathcal{S}^{p^{h}}\right)\right)$ is surjective and has kernel isomorphic to $T\left(\bar{Q}\left(\mathbb{Z} / p \otimes \mathcal{S}^{p^{h}}\right)\right)$, which is acyclic since $\mathbb{Z} / p$ was assumed to be good. So multiplication by $p$ is a quasi-isomorphism on the complex $T\left(\bar{Q}\left(\mathbb{Q} / \mathbb{Z} \otimes \mathcal{S}^{p^{h}}\right)\right)$. On the other hand, multiplication by $p$ on $\mathbb{Q} / \mathbb{Z} \otimes \mathcal{S}^{p^{h}}$ factors over a diagonalizable functor $D$, say, by Lemma 12.5. So multiplication by $p$ on $T\left(\bar{Q}\left(\mathbb{Q} / \mathbb{Z} \otimes \mathcal{S}^{p^{h}}\right)\right)$ factors through the complex $T(\bar{Q} D)$, which is acyclic by Step 1 . Since multiplication by $p$ on $T\left(\bar{Q}\left(\mathbb{Q} / \mathbb{Z} \otimes \mathcal{S}^{p^{h}}\right)\right)$ is both a quasi-isomorphism and factors through an acyclic complex, $T\left(\bar{Q}\left(\mathbb{Q} / \mathbb{Z} \otimes \mathcal{S}^{p^{h}}\right)\right)$ must itself be acyclic, and so the group $\mathbb{Q} / \mathbb{Z}$ is good.

The $\bar{Q}$-construction and the functor $T$ commute with products. So the product of a family of good abelian groups is again good. In particular a product of any number of copies of the group $\mathbb{Q} / \mathbb{Z}$ is good. Every injective abelian group is a summand of a product of copies of $\mathbb{Q} / \mathbb{Z}$, hence injective abelian groups are good.

If $A$ is a subgroup of an abelian group $B$, then the sequence of functors

$$
0 \longrightarrow A \otimes \mathcal{S}^{p^{h}} \longrightarrow B \otimes \mathcal{S}^{p^{h}} \longrightarrow(B / A) \otimes \mathcal{S}^{p^{h}} \longrightarrow 0
$$

is exact. Since the cubical construction and the functor $T$ are also exact, $A$ is good as soon as $B$ and $B / A$ are. Since an arbitrary abelian group can be embedded into an injective abelian group with injective cokernel, every abelian group is good.

\section{Step 3 Reformulation in terms of exterior power functors}

Let $\Lambda^{p^{h}} \in \mathcal{F}$ denote the exterior power functor of degree $p^{h}$. The Koszul complex (see [25, 4.3.1.7] or [17, 3.2]) is an extension of length $p^{h}-1$ of the functor $\mathbb{Z} / p \otimes \Lambda^{p^{h}}$ by the functor $\mathbb{Z} / p \otimes \mathcal{S}^{p^{h}}$ with the special property that all functors occurring in the extension are diagonalizable. By Step 1, all these intermediate terms are sent to acyclic complexes by $T(\bar{Q}-)$. Since both $T$ and 
$\bar{Q}$ are exact, the complex $T\left(\bar{Q}\left(\mathbb{Z} / p \otimes \mathcal{S}^{p^{h}}\right)\right)$ is acyclic if and only if the complex $T\left(\bar{Q}\left(\mathbb{Z} / p \otimes \Lambda^{p^{h}}\right)\right)$ is acyclic.

The spareness assumption 12.3 (c) on the cohomology of $T\left(\mathcal{S}^{p^{h}}\right)$ and the short exact sequence of functors

$$
0 \longrightarrow \mathcal{S}^{p^{h}} \stackrel{\times p}{\longrightarrow} \mathcal{S}^{p^{h}} \longrightarrow \mathbb{Z} / p \otimes \mathcal{S}^{p^{h}} \longrightarrow 0
$$

imply that the cohomology of the complex $T\left(\mathbb{Z} / p \otimes \mathcal{S}^{p^{h}}\right)$ is concentrated in dimensions congruent to 0 or $1 \bmod 2 p^{h}$. The existence of the Koszul complex then shows that the cohomology of the complex $T\left(\mathbb{Z} / p \otimes \Lambda^{p^{h}}\right)$ is concentrated in dimensions congruent to $p^{h}-1$ or $p^{h} \bmod 2 p^{h}$.

Now we set up an induction on the exponent $h$. For the inductive step we use a certain complex of functors which relates the exterior power functor of degree $p^{h}$ to that of degree $p^{h-1}$. Here and in what follows we extend the cubical construction to bounded below chain complexes of functors by applying the functor $Q$ dimensionwise and taking the total complex of the resulting bicomplex. This extended $Q$-construction is still exact and preserves quasiisomorphisms.

\section{Step 4 There exists a complex $C$ of functors from $\mathcal{F}$, concentrated in non-negative dimensions, with the following properties:}

(a) In dimension zero, $C_{0}=\mathbb{Z} / p \otimes \Lambda^{p^{h}}$ and the inclusion $\mathbb{Z} / p \otimes \Lambda^{p^{h}} \longrightarrow C$ induces quasi-isomorphisms

$$
Q\left(\mathbb{Z} / p \otimes \Lambda^{p^{h}}\right) \longrightarrow Q C \quad \text { and } \quad T\left(\mathbb{Z} / p \otimes \Lambda^{p^{h}}\right) \longrightarrow T(C) .
$$

(b) All non-trivial homology functors of $C$ are isomorphic to $\mathbb{Z} / p \otimes \Lambda^{p^{h-1}}$.

We let $\mathcal{F}\left(\mathbb{F}_{p}\right)$ denote the category of reduced functors from finitely generated $\mathbb{F}_{p}$-vector spaces to $\mathbb{F}_{p}$-vector spaces. We first construct a complex in the category $\mathcal{F}\left(\mathbb{F}_{p}\right)$; the desired complex is then obtained by composition with

$$
\left.\left.-\otimes \mathbb{F}_{p}: \text { (f.g. free abelian groups }\right) \longrightarrow \text { (f.g. } \mathbb{F}_{p}-\text { vector spaces }\right)
$$

and the inclusion of $\mathbb{F}_{p}$-vector spaces into abelian groups.

For every $\mathbb{F}_{p}$-vector space $V$ let $L^{*}(V)$ denote the quotient of the symmetric algebra on $V$ by the ideal generated by all $p$-th powers of elements. Then $L^{*}(V)$ inherits the grading from the symmetric algebra and we let $L^{n}(V)$ denote the summand of homogenous degree $n$. If $p=2$, then the functors $L^{n}$ coincide 
with the exterior power functors $\Lambda^{n}$. By [16, 1.3.1] there exists a complex $X$ (a quotient of the deRham complex) with

$$
X_{i}= \begin{cases}L^{i} \otimes \Lambda_{\mathbb{F}_{p}}^{p^{h}-i} & \text { for } 0 \leq i \leq p^{h} \\ 0 & \text { else. }\end{cases}
$$

whose only non-trivial homology is in dimension $p^{h-1}(p-1)$ where we have

$$
H_{p^{h-1}(p-1)} X \cong \Lambda_{\mathbb{F}_{p}}^{p^{h-1}}
$$

(we have reversed the grading of [16, 1.3.1] so that the differential decreases the dimension and $X$ is a chain complex as opposed to a cochain complex). The complex $X$ is part of the complex we are looking for, and we obtain the other part by dualization as follows.

The dual $D F$ of a functor $F \in \mathcal{F}\left(\mathbb{F}_{p}\right)$ is defined by $D F(V)=F\left(V^{\vee}\right)^{\vee}$, where $V^{\vee}$ refers to the dual vector space of $V$. DF is again an object of the category $\mathcal{F}\left(\mathbb{F}_{p}\right)$. Dualization is contravariant and exact in $F$ and it satisfies $D(F \otimes G) \cong D F \otimes D G$. The exterior power functors and the functors $L^{n}$ are self-dual, ie, there are isomorphisms $D \Lambda_{\mathbb{F}_{p}}^{n} \cong \Lambda_{\mathbb{F}_{p}}^{n}$ and $D L^{n} \cong L^{n}$. So if we dualize the complex $X$ we obtain a complex $D X$ which is concentrated in dimensions $-p^{h}$ through 0 , which satisfies $(D X)_{0} \cong \Lambda_{\mathbb{F}_{p}}^{p^{h}},(D X)_{-p^{h}} \cong L^{p^{h}}$ and whose only non-trivial homology is

$$
H_{-p^{h-1}(p-1)} D X \cong D \Lambda_{\mathbb{F}_{p}}^{p^{h-1}} \cong \Lambda_{\mathbb{F}_{p}}^{p^{h-1}}
$$

The desired complex $C$ is now obtained by splicing infinitely many copies of the complexes $X$ and $D X$ alternatingly at $L^{p^{h}}$ and $\Lambda_{\mathbb{F}_{p}}^{p^{h}}$, and then passing from the complex in $\mathcal{F}\left(\mathbb{F}_{p}\right)$ to a complex in $\mathcal{F}$. More precisely,

$$
\begin{aligned}
C_{0} & =\Lambda_{\mathbb{F}_{p}}^{p^{h}} \\
C_{n\left(p^{h}-1\right)+i} & = \begin{cases}X_{i} & \text { for } 1 \leq i \leq p^{h}-1 \text { and } n \geq 0 \text { even, } \\
D X_{p^{h}-i} & \text { for } 1 \leq i \leq p^{h}-1 \text { and } n \geq 0 \text { odd. }\end{cases}
\end{aligned}
$$

The map $Q\left(\mathbb{Z} / p \otimes \Lambda^{p^{h}}\right) \longrightarrow Q C$ : Let $C / C_{0}$ denote the quotient complex which is equal to $C$ except in dimension zero, where it is trivial. The complex $C / C_{0}$ is bounded below and consists entirely of diagonalizable functors. Since the $Q$-construction of a diagonalizable functor is acyclic $8.9(\mathrm{e}))$, the complex $Q\left(C / C_{0}\right)$ is also acyclic. Since $Q$ is exact, the map $Q\left(\mathbb{Z} / p \otimes \Lambda^{p^{h}}\right) \longrightarrow Q C$ is a quasi-isomorphism.

The map $T\left(\mathbb{Z} / p \otimes \Lambda^{p^{h}}\right) \longrightarrow T(C)$ : The fact that this map is a quasi-isomorphism is a consequence of the sparseness assumption 12.3 (c) on $T$. In more 
detail: the complex $C / C_{0}$ can be written as the inverse limit of the tower of truncated complexes $K^{n}$ defined by

$$
\left(K^{n}\right)_{i}= \begin{cases}C_{i} & \text { if } 1 \leq i \leq 2 n\left(p^{h}-1\right), \\ \mathbb{Z} / p \otimes \Lambda^{p^{h}} & \text { if } i=2 n\left(p^{h}-1\right)+1, \\ 0 & \text { else. }\end{cases}
$$

The boundary maps of $K^{n}$ are those of $C$ and the inclusion of the cycles

$$
\mathbb{Z} / p \otimes \Lambda^{p^{h}} \cong D\left(X_{0}\right) \longrightarrow D\left(X_{1}\right)=C_{2 n\left(p^{h}-1\right)} .
$$

The chain map $K^{n+1} \longrightarrow K^{n}$ is the identity up to dimension $2 n\left(p^{h}-1\right)$ and the epimorphism

$$
C_{2 n\left(p^{h}-1\right)+1}=X_{1} \longrightarrow X_{0}=\mathbb{Z} / p \otimes \Lambda^{p^{h}} .
$$

in dimension $2 n\left(p^{h}-1\right)+1$.

For each $n$, the kernel of the projection $K^{n} \longrightarrow\left(\mathbb{Z} / p \otimes \Lambda^{p^{h}}\right)\left[2 n\left(p^{h}-1\right)+1\right]$ onto the top functor is a bounded complex of diagonalizable functors. Since $T$ is exact and takes diagonalizable functors to acyclic complexes, the induced map

$$
T\left(K^{n}\right) \stackrel{\sim}{\longrightarrow} T\left(\left(\mathbb{Z} / p \otimes \Lambda^{p^{h}}\right)\left[2 n\left(p^{h}-1\right)+1\right]\right)
$$

is a quasi-isomorphism.

By the sparseness assumptions and Step 3, the cohomology of $T\left(\mathbb{Z} / p \otimes \Lambda^{p^{h}}\right)$ is concentrated in dimensions congruent to $p^{h}-1$ and $p^{h} \bmod 2 p^{h}$. Hence the cohomology of $T\left(K^{n}\right)$ is concentrated in dimensions congruent to $-2 n+p^{h}$ and $-2 n+p^{h}+1 \bmod 2 p^{h}$. Hence the surjective map $T\left(K^{n+1}\right) \longrightarrow T\left(K^{n}\right)$ induces trivial maps on cohomology groups for dimensional reasons. Since $T$ commutes with inverse limits, $T\left(C / C_{0}\right)$ is the inverse limit of the tower of complexes $T\left(K^{n}\right)$ for $n \geq 1$, so $T\left(C / C_{0}\right)$ is acyclic.

Example 12.6 It might be instructive to describe the complex $C$ just constructed in the smallest non-trivial case, namely for $p=2$ and $h=1$. Then $C$ is the mod 2 reduction of an 'integral' complex $\widetilde{C}$ defined by

$$
\widetilde{C}_{i}= \begin{cases}0 & \text { if } i<0, \\ \Lambda^{2} & \text { if } i=0, \text { and } \\ I \otimes I & \text { if } i>0 .\end{cases}
$$

The differential $d_{i}: \widetilde{C}_{i} \longrightarrow \widetilde{C}_{i-1}$ is given by

$$
d_{i}(x \otimes y)= \begin{cases}x \wedge y & \text { if } i=1 \\ x \otimes y+y \otimes x & \text { if } i \geq 2 \text { and } i \text { is even, } \\ x \otimes y-y \otimes x & \text { if } i \geq 2 \text { and } i \text { is odd }\end{cases}
$$


The homology of the complex $\widetilde{C}$ is given by

$$
H_{i} \widetilde{C} \cong \begin{cases}\mathbb{Z} / 2 \otimes I & \text { if } i \geq 1 \text { and } i \text { is odd } \\ 0 & \text { else. }\end{cases}
$$

Since the homology functors of $\widetilde{C}$ are additive and since $\widetilde{C}$ is diagonalizable in positive dimensions, $\widetilde{C}$ is quasi-isomorphic to the cubical construction $Q \Lambda^{2}$. Hence by 8.9 (b) we can read off the Dold-Puppe stable derived functors of $\Lambda^{2}$ as

$$
L_{i}^{\mathrm{st}} \Lambda^{2} \cong H_{i} \widetilde{C}(\mathbb{Z}) \cong \begin{cases}\mathbb{Z} / 2 & \text { if } i \geq 1 \text { and } i \text { is odd }, \\ 0 & \text { else, }\end{cases}
$$

compare e.g. [50]. The complex $C$ constructed in Step 4 for $p=2$ and $h=1$ is isomorphic to the reduction $\mathbb{Z} / 2 \otimes \widetilde{C}$; by the universal coefficient theorem, the homology functors of $C$ are thus isomorphic to the functor $\mathbb{Z} / 2 \otimes I$ in every positive dimension, and trivial otherwise.

\section{Step 5 The complex $T\left(\bar{Q}\left(\mathbb{Z} / p \otimes \Lambda^{p^{h}}\right)\right)$ is acyclic for all $h \geq 0$.}

We proceed by induction. For $h=0$ we have $\mathbb{Z} / p \otimes \Lambda^{1}=\mathbb{Z} / p \otimes I$ which is an additive functor. Thus all cross-effect vanish and by property 8.9 (c) of the cubical construction, the complex $\bar{Q}\left(\mathbb{Z} / p \otimes \Lambda^{1}\right)$ is trivial. Hence $T\left(\bar{Q}\left(\mathbb{Z} / p \otimes \Lambda^{1}\right)\right)$ is also trivial.

Now suppose that $h \geq 1$ and assume that $T\left(\bar{Q}\left(\mathbb{Z} / p \otimes \Lambda^{p^{h-1}}\right)\right)$ is already known to be acyclic. Let $C$ be any complex as in Step 4 . We consider the commutative square of bounded below chain complexes of functors

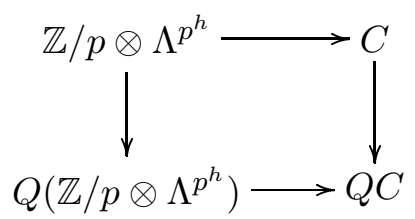

where we view the functor $\mathbb{Z} / p \otimes \Lambda^{p^{h}}$ as a complex concentrated in dimension zero and the horizontal maps are induced by the inclusion $\mathbb{Z} / p \otimes \Lambda^{p^{h}} \longrightarrow C$.

By property (a) of the complex $C$ and since $T$ preserves quasi-isomorphisms, both rows of the square induce a quasi-isomorphism after applying $T$. Since $T$ is exact, the map

$$
T\left(\bar{Q}\left(\mathbb{Z} / p \otimes \Lambda^{p^{h}}\right)\right) \longrightarrow T(\bar{Q} C)
$$

is thus a quasi-isomorphism. So in order to finish the induction step, it remains to show that the complex $T(\bar{Q} C)$ is acyclic. 
This last step is where the induction hypothesis is used. The complex $C$ is the inverse limit of its Postnikov tower (homological truncations)

$$
\cdots \longrightarrow P_{n} \longrightarrow \cdots \longrightarrow P_{1} \longrightarrow P_{0} \longrightarrow P_{-1}=0 \text {. }
$$

In the tower each map $P_{n} \longrightarrow P_{n-1}$ is a surjection whose kernel is quasiisomorphic to the $n$-th homology functor of $C$ concentrated in dimension $n$. By property (b) of the complex $C$ all non-trivial homology functors are isomorphic to the exterior power functor $\mathbb{Z} / p \otimes \Lambda^{p^{h-1}}$, for which we already know that the map $T\left(\bar{Q}\left(\mathbb{Z} / p \otimes \Lambda^{p^{h-1}}\right)\right)$ is acyclic. Since the $Q$-construction and $T$ are exact we conclude by induction that for all $n \geq 0$ the complex $T\left(\bar{Q} P_{n}\right)$ is acyclic.

The Postnikov tower consists of bounded below chain complexes and it stabilizes in each dimension. So the complex $\bar{Q} C$ is the inverse limit of the complexes $\bar{Q} P_{n}$. Since $T$ commutes with the inverse limits, $T(\bar{Q} C)$ is the inverse limits of the acyclic complexes $T\left(\bar{Q} P_{n}\right)$. Since $T$ and $\bar{Q}$ also preserve epimorphisms, this inverse limit is acyclic.

\section{Perspectives}

We end the paper with an application of Theorem 5.2 which concerns an interesting homotopical property of the Gamma-ring $D B$. Then we discuss some variants of the Construction 3.5 of Gamma-ring maps from formal group laws, and some possible directions for further investigation.

For the application we use the conjugation action to obtain an obstruction to the existence of $k$-algebra structures on Gamma-rings. With this tool we then show that the Gamma-ring $D B$ is not stably equivalent to the EilenbergMacLane Gamma-ring of any simplicial ring (unless $B$ is a $\mathbb{Q}$-algebra). To motivate the criterion we look at the classical case of discrete rings first. If $k$ is a commutative ring and $A$ any associative ring, then the $k$-algebra structures on $A$ correspond to the central ring maps $k \longrightarrow A$. In particular, the unit map of every such $k$-algebra structure gives an element of the set $\operatorname{Ring}(k, A)$ of ring maps which is a fixed point of the conjugation action of the units of $A$. Something similar happens for Gamma-rings. Suppose $R$ is a Gamma-ring which is stably equivalent to an algebra over the commutative Gamma-ring $k$. Any chain of equivalences to a $k$-algebra determines a homotopy class of Gamma-ring maps $[\eta] \in[k, R]_{\text {Ho } \mathcal{G R}}$ underlying the unit map of the algebra structure. 
Theorem 13.1 Suppose $\eta \in \operatorname{Ring}(k, R)$ is a Gamma-ring map whose homotopy class underlies a $k$-algebra structure of $R$. Then the conjugation action map

$$
U R^{\times} \longrightarrow \operatorname{Ring}(k, R) \quad, \quad u \mapsto u \cdot \eta \cdot u^{-1}
$$

is null-homotopic. So if the conjugation action map is essential for every component of the space $\operatorname{Ring}(k, R)$ of Gamma-ring maps, then $R$ is not stably equivalent to any $k$-algebra.

Proof We can assume that $R$ is itself a stably fibrant $k$-algebra. Then Construction 4.2 can be done in the category of $k$-algebras, as opposed to Gammarings, relative to the trivial group (Lemma 4.4 is also valid in the category of $k$-algebras). We obtain a diagram of $k$-algebras

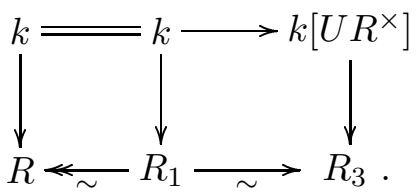

Ignoring the $k$-algebra structure we can use the objects in this diagram to model the space of Gamma-ring maps from $k$ to $R$. More precisely, the space $\operatorname{hom}_{\mathcal{G R}}\left(k^{\mathrm{c}}, R_{3}\right)$ admits a conjugation action by the simplicial group $U R^{\times}$and this action is equivalent to the one in question (here $k^{\mathrm{c}}$ is a cofibrant replacement of $k$ as a Gamma-ring). But in this model for the conjugation action, the composite of the approximation map $k^{\mathrm{c}} \longrightarrow k$ with the unit map $k \longrightarrow R_{3}$ of the $k$-algebra structure on $R_{3}$ is a point-set level fixed point of the conjugation action of $U R^{\times}$.

If we combine the previous result with Theorem 5.2 we can deduce that the Gamma-ring $D B$ is not stably equivalent to the Eilenberg-MacLane Gammaring of any simplicial ring, unless $B$ is an algebra over the rational numbers. This should be compared to Theorem 3.2 (b) which says that as a $\Gamma$-space, $D B$ is stably equivalent to the smash product $H \mathbb{Z} \wedge^{L} H B$. In other words, $D B$ 'additively' decomposes into a product of Eilenberg-MacLane $\Gamma$-spaces. In contrast the following corollary shows that the multiplicative structure of $D B$ is genuinely homotopy-theoretic. The idea of the proof is that any stable equivalence between $D B$ and a simplicial ring would give $D B$ the structure of an $H \mathbb{Z}$-algebra. Such an algebra structure in turn gives rise to a "central" Gamma-ring map $H \mathbb{Z} \longrightarrow D B$ (in the sense of Theorem 13.1). But Theorem 5.2 identifies all Gamma-ring map from $H \mathbb{Z}$ to $D B$ and shows that none of them is "central". 
Formal groups and stable homotopy of commutative rings

Corollary 13.2 For a commutative ring $B$ the following conditions are equivalent.

(1) $B$ is an algebra over the rational numbers.

(2) The projection $D B \longrightarrow D_{1} B \cong H B$ is a stable equivalence of Gammarings.

(3) $D B$ is stably equivalent (as a Gamma-ring) to the Eilenberg-MacLane Gamma-ring of a simplicial ring.

Proof Condition (1) is equivalent to condition (2) by Theorem 3.2 (b), and condition (2) implies condition (3). The proof that condition (3) implies condition (2) is a combination of Theorem 13.1 in the case $k=H \mathbb{Z}$, with Theorem 5.2. Assume that condition (3) holds. Since the Eilenberg-MacLane Gammaring of a simplicial ring is an $H \mathbb{Z}$-algebra, there exist a component of the space $\operatorname{Ring}(H \mathbb{Z}, D B)$ of Gamma-ring maps for which the conjugation action map $D B^{\times} \longrightarrow \operatorname{Ring}(H \mathbb{Z}, D B)$ is homotopically trivial, by Theorem 13.1. But this map is part of a homotopy fiber sequence

$$
(D B)_{1}^{\times} \longrightarrow \operatorname{Ring}(H \mathbb{Z}, D B) \longrightarrow \operatorname{Ring}(H \mathbb{Z}, D B) / \text { conj. }
$$

Since the base of this fibration is weakly equivalent to the classifying space of a groupoid (Theorem 5.2), its homotopy groups are trivial above dimension 1. So the conjugation action map is injective on homotopy groups in positive dimensions. Since the map is also null-homotopic, the space $(D B)_{1}^{\times}$must be weakly contractible, which implies condition (2).

13.3 Coordinate free definition The definition of the Gamma-ring $D B$ and the Gamma-ring map $F_{*}$ depended on a formal group law $F$, ie, on a 1-dimensional commutative formal group with a choice of coordinate. We will now describe coordinate-free versions of these constructions which at the same time are defined in a more general context.

As input we consider a category $\mathcal{C}$ which has a zero object and finite coproducts. The natural enrichment of $\mathcal{C}$ over the category $\Gamma^{\mathrm{op}}$ of finite pointed sets is given by

$$
X \wedge k^{+}=\underbrace{X \amalg \cdots \amalg X}_{k} \quad(\text { coproduct in } \mathcal{C}) .
$$

Every object $X$ of $\mathcal{C}$ has an endomorphism Gamma-ring [46, 4.6], denoted $\operatorname{End}_{\mathcal{C}}(X)$ and defined by

$$
\operatorname{End}_{\mathcal{C}}(X)\left(k^{+}\right)=\operatorname{Hom}_{\mathcal{C}}\left(X, X \wedge k^{+}\right) .
$$


The unit map $\mathbb{S} \longrightarrow \operatorname{End}_{\mathcal{C}}(X)$ comes from the identity map of $X$, viewed as a point in $\operatorname{End}_{\mathcal{C}}(X)\left(1^{+}\right)$, and the multiplication $\operatorname{End}_{\mathcal{C}}(X) \wedge \operatorname{End}_{\mathcal{C}}(X) \longrightarrow$ $\operatorname{End}_{\mathcal{C}}(X)$ is induced by the composition product

$$
\begin{aligned}
\operatorname{End}_{\mathcal{C}}(X)\left(k^{+}\right) & \wedge \operatorname{End}_{\mathcal{C}}(X)\left(l^{+}\right) \\
f \wedge g & \longmapsto \operatorname{End}_{\mathcal{C}}(X)\left(k^{+} \wedge l^{+}\right), \\
& \longmapsto\left(f \wedge l^{+}\right) \circ g .
\end{aligned}
$$

As an example we can take $\mathcal{C}$ to be the category of commutative, complete augmented $B$-algebras. If we choose the object $X$ to be the power series ring on one generator, then the endomorphism Gamma-ring of $X$ is precisely $D B$.

Now we suppose that the object $X$ of $\mathcal{C}$ is equipped with the structure of abelian cogroup object. So there is a given co-addition map $X \longrightarrow X \amalg X$ and a co-inverse map $X \longrightarrow X$ which make the set $\operatorname{Hom}_{\mathcal{C}}(X, Y)$ into an abelian group, natural for all objects $Y$ of $\mathcal{C}$. Every abelian cogroup structure on $X$ gives rise to a homomorphism of Gamma-rings $H \mathbb{Z} \longrightarrow \operatorname{End}_{\mathcal{C}}(X)$ as follows. At a finite pointed set $k^{+}$the map

$$
H \mathbb{Z}\left(k^{+}\right)=\widetilde{\mathbb{Z}}\left[k^{+}\right] \longrightarrow \operatorname{Hom}_{\mathcal{C}}\left(X, X \wedge k^{+}\right)=\operatorname{End}_{\mathcal{C}}(X)\left(k^{+}\right)
$$

is the additive extension of the map that sends $i \in k^{+}$to the $i$-th coproduct inclusion $X \longrightarrow X \wedge k^{+}$. When $\mathcal{C}$ is the category of commutative, complete augmented $B$-algebras and $X$ is the power series ring on one generator, then making $X$ into an abelian cogroup object is the same thing as giving a (1dimensional, commutative) formal group law $F$ over $B$. Furthermore, in this case the map $H \mathbb{Z} \longrightarrow \operatorname{End}_{\mathcal{C}}(X)$ arising from the abelian cogroup structure corresponds to the map $F_{*}$ of Construction 3.5 under the identification $D B \cong$ $\operatorname{End}_{\mathcal{C}}(X)$. So from this point of view construction 3.5 is just a special case of the fact that every abelian cogroup structures gives rise to a homomorphism from the Eilenberg-MacLane Gamma-ring $H \mathbb{Z}$ to an endomorphism Gamma-ring.

13.4 Non-commutative formal group laws Construction 3.5 can be modified to work for not necessarily commutative formal group laws, but this variant does not lead to any interesting phenomena. There is a Gamma-ring, denoted by $G p$, which is constructed the same way $H \mathbb{Z}$ is, but with free groups instead of free abelian groups. So as a $\Gamma$-space, $G p$ takes a pointed set to the reduced free group it generates. The multiplication again comes from substitution, this times words in the generators of the free groups are substituted into each other. Abelianization gives a Gamma-ring map $G p \longrightarrow H \mathbb{Z}$. If $F$ is a 1 -dimensional but not necessarily commutative formal group law over the commutative ring $B$, then it gives rise to a Gamma-ring map

$$
F_{*}: G p \longrightarrow D B
$$


in much the same way as in Construction 3.5. The Gamma-ring map $F_{*}$ factors over $H \mathbb{Z}$ if and only if the formal group law $F$ is commutative.

While the construction makes sense, $G p$ is uninteresting as a source of Gammaring homomorphisms: we claim that the unit map $\mathbb{S} \longrightarrow G p$ from the sphere Gamma-ring is a stable equivalence. This claim follows from the fact that the map from a high dimensional sphere into the free group it generates is an equivalence in the stable range. Since the Gamma-ring $G p$ is stably equivalent to the initial Gamma-ring, the derived space of homomorphisms into any other Gamma-ring is contractible.

13.5 Higher dimensional formal group laws Another variant of Construction 3.5 proceeds from an $n$-dimensional commutative formal group law $F$. This time the construction gives a weak Gamma-ring map

$$
F_{*}: H \mathbb{Z} \longrightarrow M_{n}(D B)
$$

into the Gamma-ring of $n \times n$-matrices over $D B$. For an arbitrary Gammaring $R$ the Gamma-ring $M_{n}(R)$ of $n \times n$-matrices over $R$ is defined as the endomorphism Gamma-ring of the free $R$-module on $n$ generators, ie,

$$
M_{n}(R)=\operatorname{Hom}_{R-\bmod }\left(R \wedge n^{+}, R \wedge n^{+}\right)
$$

(here $\operatorname{Hom}_{R}$ refers to the internal homomorphism $\Gamma$-space in the category of $R$-modules). We define another Gamma-ring $\bar{M}_{n}(D B)$ as the endomorphism Gamma-ring, in the sense of 13.3, of the power series ring in $n$ generators in the category of augmented, complete $B$-algebras. Then there is a stable equivalence $M_{n}(D B) \longrightarrow \bar{M}_{n}(D B)$. Since an $n$-dimensional commutative formal group law $F$ is the same thing as an abelian cogroup structure on the power series ring in $n$ variables, it leads to a map of Gamma-rings $F_{*}: H \mathbb{Z} \longrightarrow \bar{M}_{n}(D B)$.

13.6 Formal module structures Yet another variation of our main theme consists in considering formal module structures over an associative ring $R$. A (1-dimensional) formal $R$-module (law) over a commutative ring $B$ consists of a (1-dimensional and commutative) formal group law $F$ and a ring homomorphism from $R$ into the endomorphism ring of the formal group law $F$. In the spirit of Construction 3.5. any formal $R$-module structure $F$ over $B$ gives rise to a Gamma-ring map $F_{*}: H R \longrightarrow D B$ with source the Eilenberg-MacLane Gamma-ring of $R$.

By the same method as in Section [5] we obtain a map

$$
\kappa_{R}: \widetilde{\mathcal{F} R \text {-mod }}^{\text {str }}(B) \longrightarrow \operatorname{Ring}(H R, D B) / \text { conj. }
$$


from the groupoid of formal $R$-module structures over $B$ and strict isomorphisms to the homotopy orbits of the derived space of Gamma-ring maps by the connected component of the homotopy units $D B^{\times}$. It seems reasonable to expect that the map $\kappa_{R}$ is again a weak equivalence; whether this is the case depends on whether the appropriate analog of Theorem 12.1 holds over the ring $R$.

We denote by $R^{\mathrm{com}}=R /(r s-s r)$ the quotient of $R$ by the commutator ideal. Then the arguments of Sections [6] through [1] can be adapted to show:

Theorem 13.7 Suppose that the ring $R$ has the following property: for all $m \in \mathbb{Z}$, all $k \geq 0$ and $R^{\text {com }}$-modules $A$ the map

$$
\operatorname{Ext}_{\mathcal{F}\left(R^{\mathrm{com}}\right)}^{m}\left(I, A \otimes_{R^{\mathrm{com}}} \mathcal{S}^{k}\right) \longrightarrow \mathbb{E x t}_{\mathcal{F}\left(R^{\mathrm{com}}\right)}^{m}\left(I, Q\left(A \otimes_{R^{\mathrm{com}}} \mathcal{S}^{k}\right)\right)
$$

is an isomorphism. Then the map

$$
\kappa_{R}: \widetilde{\mathcal{F R} \text {-mod }}^{\text {str }}(B) \longrightarrow \operatorname{Ring}(H R, D B) / \text { conj. }
$$

is a weak equivalence of simplicial sets.

The hypothesis is true for $R=\mathbb{Z}$ - this is the content of Theorem 12.1. The proof of Theorem 12.1 can be adapted to establish the hypothesis for $R=\mathbb{Z} / n$. We conjecture that indeed the hypothesis holds in general; we expect that a 'good' proof of Theorem 12.1 ie, a proof that does not use the calculations of the MacLane cohomology groups $\operatorname{Ext}_{\mathcal{F}}^{*}\left(I, \mathcal{S}^{k}\right)$ as input, would also work in the more general context. If this is the case, the map $\kappa_{R}$ is a weak equivalence for any ring $R$.

Example 13.8 Suppose $B$ is an $\mathbb{F}_{p}$-algebra and $F$ a formal group law over $F$. In this case we can reinterpret the height of $F$ in terms of the homotopy class of the Gamma-ring map $F_{*}$.

The $p$-series of $F$ is either trivial or of the form

$$
[p]_{F}(x)=u \cdot x^{p^{h}}+\text { terms of higher degree }
$$

for some $h \geq 1$ and some non-zero $u \in B$. The number $h$ is called the height of $F$. If $[p]_{F}=0$, then $F$ is isomorphic to the additive formal group law [19, III.1 Cor. 2], and the height of $F$ is infinite.

Claim The height of $F$ is equal to the largest number $h$ such that

$$
H \mathbb{Z} \stackrel{F_{*}}{\longrightarrow} D B \longrightarrow D_{p^{h}-1} B
$$


can be factored, in the homotopy category of Gamma-rings, over the EilenbergMacLane Gamma-ring for $\mathbb{F}_{p}$.

Indeed, if $F$ has height $h$, then for every pointed set $K$ the map

$$
F_{*}(K): \widetilde{\mathbb{Z}}[K]=H \mathbb{Z}(K) \longrightarrow D B(K) \subseteq \widetilde{B} \llbracket K \rrbracket
$$

satisfies

$$
F_{*}(p \cdot x)=[p]_{F}\left(F_{*}(x)\right) \equiv 0 \quad \text { modulo degree } p^{h} .
$$

So the composite map

$$
H \mathbb{Z} \stackrel{F_{*}}{\longrightarrow} D B \longrightarrow D_{p^{h-1}} B
$$

factors uniquely over $H \mathbb{F}_{p}$ on the point-set level. Conversely, suppose that there exists a commutative square in the homotopy category of Gamma-rings

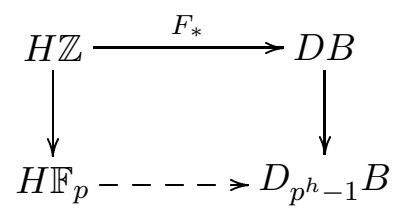

By the analog of Theorem 6.4 for buds of formal $\mathbb{F}_{p}$-modules (which holds since the hypothesis of Theorem 13.7 are satisfied for $R=\mathbb{F}_{p}$ ), the maps from $H \mathbb{F}_{p} \longrightarrow D_{p^{h}-1} B$ in the homotopy category of Gamma-rings are in bijective correspondence with strict isomorphism classes of $p^{h}$-buds of formal $\mathbb{F}_{p}$-module structures on $B$. But over an $\mathbb{F}_{p}$-algebra every formal $\mathbb{F}_{p}$-module bud is strictly isomorphic to the additive formal group law [19, III.1 Cor. 2]. Hence the $\left(p^{h}-\right.$ 1 )-buds of $F$ and of the additive formal group law over $B$ induce the same maps $H \mathbb{Z} \longrightarrow D_{p^{h}-1} B$ in the homotopy category of Gamma-rings. By Theorem 6.4 the $\left(p^{h}-1\right)$-bud of $F$ is thus strictly isomorphic to the $\left(p^{h}-1\right)$-bud of the additive formal group law, and so $[p]_{F} \equiv 0$ modulo degree $p^{h}-1$. So the height of $F$ is at least $h$.

\section{References}

[1] M Basterra, André-Quillen cohomology of commutative S-algebras, J. Pure Appl. Algebra 144 (1999) 111-143

[2] M Basterra, R McCarthy, Г-homology, topological André-Quillen homology and stabilization, Topology Appl. 121 (2002) 551-566 
[3] M Basterra, B Richter, (Co-)homology theories for commutative (S-)algebras, from: "Structured Ring Spectra", (A Baker, B Richter, editors), to appear in London Mathematical Society Lecture Notes, Cambridge University Press

[4] S Betley, T Pirashvili, Stable K-theory as a derived functor, J. Pure Appl. Algebra 96 (1994) 245-258

[5] M Bökstedt, Topological Hochschild homology (1985), preprint, Bielefeld

[6] F Borceux, Handbook of Categorical Algebra 2: Categories and Structures, volume 51 of Encyclopedia of Mathematics and its Applications, Cambridge University Press (1994)

[7] A K Bousfield, Operations on Derived Functors of Non-additive Functors (1967), mimeographed Notes, Brandeis University

[8] A K Bousfield, E M Friedlander, Homotopy theory of $\Gamma$-spaces, spectra, and bisimplicial sets, from: "Geometric Applications of Homotopy Theory II", Lecture Notes in Mathematics 658, Springer (1978) 80-130

[9] A K Bousfield, D M Kan, Homotopy limits, completions and localizations, volume 304 of Lecture Notes in Mathematics, Springer (1972)

[10] H Cartan, Algébres d'Eilenberg-MacLane et homotopie (1954-55), seminaire Henri Cartan

[11] A Dold, D Puppe, Homologie nicht-additiver Funktoren. Anwendungen, Ann. Inst. Fourier Grenoble 11 (1961) 201-312

[12] W G Dwyer, Homotopy operations for simplicial commutative algebras, Trans. Amer. Math. Soc. 260 (1980) 421-435

[13] W G Dwyer, J Spalinski, Homotopy theories and model categories, from: "Handbook of algebraic topology", (I M James, editor), North-Holland, Amsterdam (1995) 73-126

[14] S Eilenberg, S MacLane, Homology theories for multiplicative systems, Trans. Amer. Math. Soc. 71 (1951) 294-330

[15] S Eilenberg, S MacLane, On the groups $H(\pi, n)$, II, Ann. Math. 60 (1954) 49-139

[16] V Franjou, Extensions entre puissances extérieures et entre puissances symétriques, J. Algebra 179 (1996) 501-522

[17] V Franjou, J Lannes, L Schwartz, Autour de la cohomologie de Mac Lane des corps finis, Invent. Math. 115 (1994) 513-538

[18] V Franjou, T Pirashvili, On the MacLane cohomology for the ring of integers, Topology 37 (1998) 109-114

[19] A Fröhlich, Formal groups, volume 74 of Lecture Notes in Mathematics, Springer-Verlag, Berlin (1968)

[20] P G Goerss, A Hilton-Milnor theorem for categories of simplicial algebras, Amer. J. Math. 111 (1989) 927-971 
Formal groups and stable homotopy of commutative rings

[21] P G Goerss, On the André-Quillen cohomology of commutative $\mathbb{F}_{2}$-algebras, Asterisque 186 (1990)

[22] P G Goerss, J F Jardine, Simplicial homotopy theory, Birkhäuser Verlag, Basel (1999)

[23] M Hovey, Model categories, American Mathematical Society, Providence, RI (1999)

[24] M Hovey, B E Shipley, J H Smith, Symmetric spectra, J. Amer. Math. Soc. 13 (2000) 149-208

[25] L Illusie, Complexe cotangent et déformations. I, volume 239 of Lecture Notes in Mathematics, Springer-Verlag, Berlin (1971)

[26] M Jibladze, T Pirashvili, Cohomology of algebraic theories, J. Algebra 137 (1991) 253-296

[27] B Johnson, R McCarthy, Linearization, Dold-Puppe stabilization, and Mac Lane's Q-construction, Trans. Amer. Math. Soc. 350 (1998) 1555-1593

[28] M Lazard, Sur les groupes de Lie formels à un paramètre, Bull. Soc. Math. France 83 (1955) 251-274

[29] M Lydakis, Smash products and $\Gamma$-spaces, Math. Proc. Cambridge Philos. Soc. 126 (1999) 311-328

[30] S MacLane, Homologie des anneaux et des modules, from: "Colloque de topologie algébrique, Louvain, 1956", Georges Thone, Liège (1957) 55-80

[31] S MacLane, Categories for the working mathematician, Springer (1971)

[32] M A Mandell, J P May, S Schwede, B Shipley, Model categories of diagram spectra, Proc. London Math. Soc. (3) 82 (2001) 441-512

[33] D McDuff, On the classifying spaces of discrete monoids, Topology 18 (1979) 313-320

[34] T Pirashvili, Higher additivizations, Trudy Tbiliss. Mat. Inst. Razmadze Akad. Nauk Gruzin. SSR 91 (1988) 44-54

[35] T Pirashvili, Polynomial approximation of Ext and Tor groups in functor categories, Comm. Algebra 21 (1993) 1705-1719

[36] T Pirashvili, B Richter, Robinson-Whitehouse complex and stable homotopy, Topology 39 (2000) 525-530

[37] T Pirashvili, F Waldhausen, MacLane homology and topological Hochschild homology, J. Pure Appl. Algebra 82 (1992) 81-98

[38] D Quillen, On the (co-) homology of commutative rings, from: "Applications of Categorical Algebra (Proc. Sympos. Pure Math., Vol. XVII, New York, 1968)", Amer. Math. Soc., Providence, R.I. (1970) 65-87

[39] D G Quillen, Homotopical Algebra, volume 43 of Lecture Notes in Mathematics, Springer (1967)

[40] C L Reedy, Homotopy theory of model categories, preprint, 1973. 
[41] A Robinson, Gamma homology, Lie representations and $E_{\infty}$ multiplications, Invent. Math. 152 (2003) 331-348

[42] A Robinson, $\mathbf{S}$ Whitehouse, Operads and $\Gamma$-homology of commutative rings, Math. Proc. Cambridge Philos. Soc. 132 (2002) 197-234

[43] S Schwede, Spectra in model categories and applications to the algebraic cotangent complex, J. Pure Appl. Algebra 120 (1997) 77-104

[44] S Schwede, Stable homotopical algebra and $\Gamma$-spaces, Math. Proc. Cambridge Philos. Soc. 126 (1999) 329-356

[45] S Schwede, S-modules and symmetric spectra, Math. Ann. 319 (2001) 517-532

[46] S Schwede, Stable homotopy of algebraic theories, Topology 40 (2001) 1-41

[47] S Schwede, B E Shipley, Algebras and modules in monoidal model categories, Proc. London Math. Soc. (3) 80 (2000) 491-511

[48] G Segal, Categories and cohomology theories, Topology 13 (1974) 293-312

[49] B Shipley, Symmetric spectra and topological Hochschild homology, K-Theory 19 (2000) 155-183

[50] D Simson, Stable derived functors of the second symmetric power functor, second exterior power functor and Whitehead gamma functor, Colloq. Math. 32 (1974) 49-55

[51] $\mathbf{J} \mathbf{M}$ Turner, Simplicial commutative $\mathbf{F}_{p}$-algebras through the looking-glass of $\mathbf{F}_{p}$-local spaces, from: "Homotopy invariant algebraic structures (Baltimore, MD, 1998)", Amer. Math. Soc., Providence, RI (1999) 353-363 